\title{
A monoanionic pentadentate ligand platform for scandium- pnictogen multiple bonds
}

Evan A. Patrick, a Yan Yang, barren E. Piers*, a Laurent Maron*, b and Benjamin S. Gelfand. ${ }^{\mathrm{a}}$

aDepartment of Chemistry, University of Calgary, 2500 University Dr. NW, Calgary, Alberta, Canada, T2N 1N4

b LPCNO, Université de Toulouse, INSA, UPS, LPCNO, 135 avenue de Rangueil, F31077 Toulouse, France, and CNRS, LPCNO, F-31077 Toulouse, France

Electronic Supplementary Information (ESI) available: Text and Figures giving further experimental and spectroscopic details, X-ray structural data for $\mathbf{B P z}_{2} \mathbf{P y} \mathbf{y}_{3} \mathbf{H}$, 2-N, 4 and 5, (CCDC 2090428-2090431) and full details on the computational results.

\section{Abstract \\ A new monoanionic pentadentate ligand is designed to accommodate $\mathrm{Sc}=\mathrm{E}$ bonds $(E=N, P)$. The imido complex is stable enough to isolate and characterize, and reacts rapidly with $\mathrm{CO}_{2}$. The phosphinidene, on the other hand, is highly reactive and induces $\mathrm{C}-\mathrm{C}$ bond cleavage to yield a phosphido- pyridyl complex which also undergoes rapid reacton with $\mathrm{CO}_{2}$.}

The generation and reactivity of well-defined terminal group 3 metal to main group element multiple bonded compounds has been an active area of investigation both for fundamental interest and to take advantage of the high reactivity of such species in group transfer or bond activation applications. ${ }^{1-4}$ Generally, the mismatch in orbital energies for $\mathrm{M}_{\text {group } 3}=\mathrm{E}\left(\mathrm{E}=\mathrm{O}, \mathrm{NR}, \mathrm{PR}, \mathrm{CR}_{2}\right)$ complexes render the bonds highly polarized and therefore prone to oligomerization or complexation by Lewis acids, such that terminal, unsupported $\mathrm{M}=\mathrm{E}$ complexes remain rare, despite recent advances. ${ }^{5}$ Most of the examples reported focus on imido derivatives of scandium ${ }^{6-11}$ (Chart 1), since the NR group offers good steric protection and the relatively small 
size of Sc(III) mitigates against oligomerization or Lewis acid complexation by discouraging higher coordination numbers. More recently, evidence for highly reactive terminal scandium phosphinidene complexes has appeared. ${ }^{12,13}$

We have been exploring a dianionic pentadentate ligand, $\left(\mathbf{B}_{2} \mathbf{P} \mathbf{z}_{4} \mathbf{P y} \text {, Chart } 1\right)^{14}$ for the stabilization of higher oxidation state imido ${ }^{15}$ and oxo ${ }^{16}$ derivatives of the middle transition elements. While scandium complexes of this ligand are readily available, ${ }^{17-19}$ as a platform for supporting Sc=NR or other multiple bonds it mostly fails ${ }^{20}$ since the complexes are necessarily anionic due to the dianionic nature of the ligand, rendering the resulting $\mathrm{Sc}=\mathrm{E}$ complexes highly basic. We therefore have

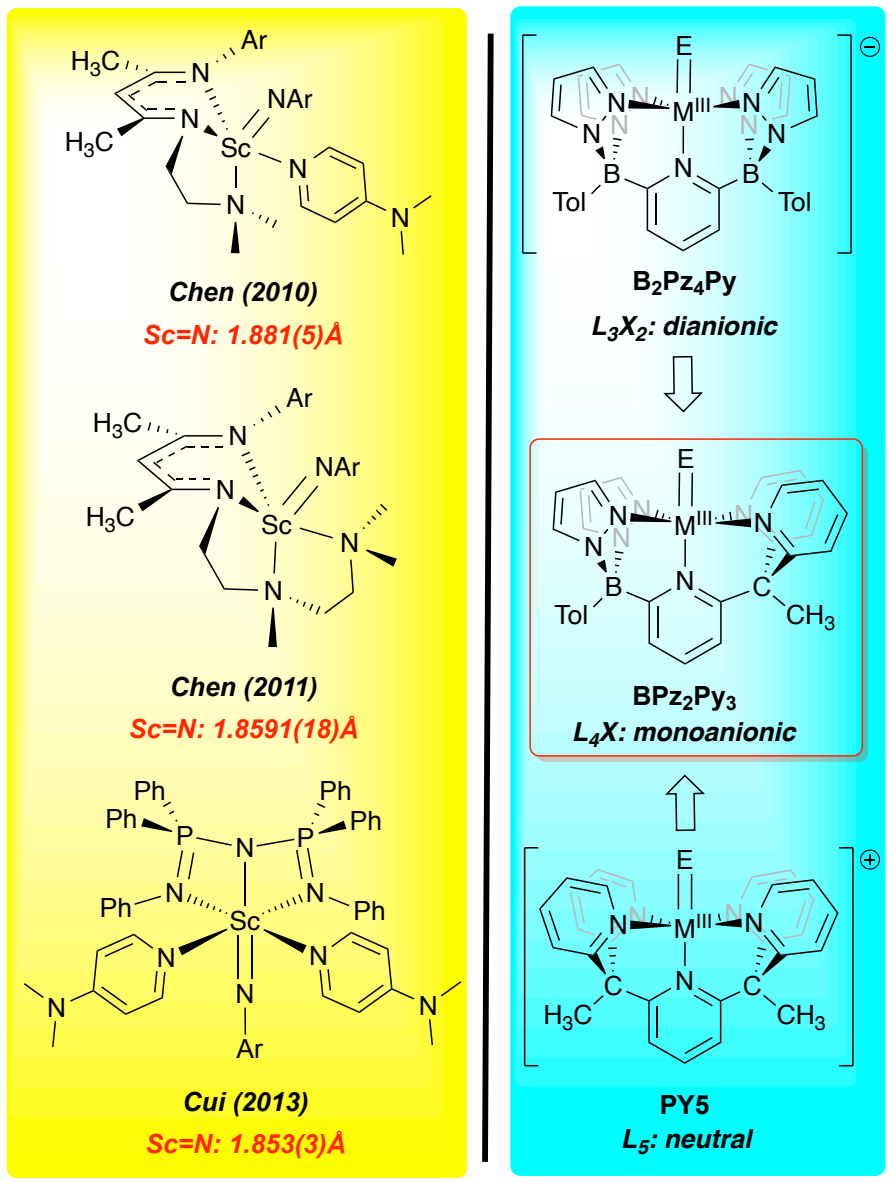

Chart 1. Left: Structurally characterized scandium imides $(\mathrm{Ar}=2,6-$ di-isopropylphenyl). Right: Conceptual genesis of monoanionic pentadentate ligand $\mathbf{B}_{2} \mathbf{P z}_{2} \mathbf{P y}_{3}$ as a platform for $\mathrm{Sc}=\mathrm{E}$ complexes ( $\mathrm{E}=\mathrm{NAr}$, PAr). 
developed a monoanionic pentadentate ligand that is a hybrid of $\mathbf{B}_{2} \mathbf{P z}_{4} \mathbf{P y}$ and the well-studied, neutral PY5 ligand ${ }^{21,22}\left(\mathbf{B P z}_{2} \mathbf{P y} \mathbf{y}_{3}\right.$, Chart 1) as a ligand more suited to stabilizing group 3 metal element multiple bonds in neutral complexes. Here we report a terminal aryl imido complex and the intermediacy of an aryl phosphinidene derivative of the new ligand $\mathbf{B P z} \mathbf{P}_{2} \mathbf{P y}_{3}$ along with preliminary reactivity studies involving carbon dioxide $\left(\mathrm{CO}_{2}\right)$.

The new proligand $\mathbf{B P z}_{2} \mathbf{P y}_{3} \mathbf{H}$ was prepared on a multigram scale as its pyridiniumborate zwitterion in 2 steps (57\%) as a faint pink solid (see Scheme S1, ESI). The pyridinium proton resonates at $17.02 \mathrm{ppm}$ in the ${ }^{1} \mathrm{H}$ NMR spectrum and a peak at $1.2 \mathrm{ppm}$ appears in the ${ }^{11} \mathrm{~B}\left\{{ }^{1} \mathrm{H}\right\}$ NMR spectrum; the compound was also characterized via X-ray crystallography (Figure S1). When treated with one equivalent of $\mathrm{Sc}\left(\mathrm{CH}_{2} \mathrm{SiMe}_{2} \mathrm{Ph}\right)_{3}(\mathrm{THF})_{2},{ }^{23}$ rapid loss of alkane gives a bright orange solution of a bis-alkyl scandium complex tentatively assigned as the octahedral

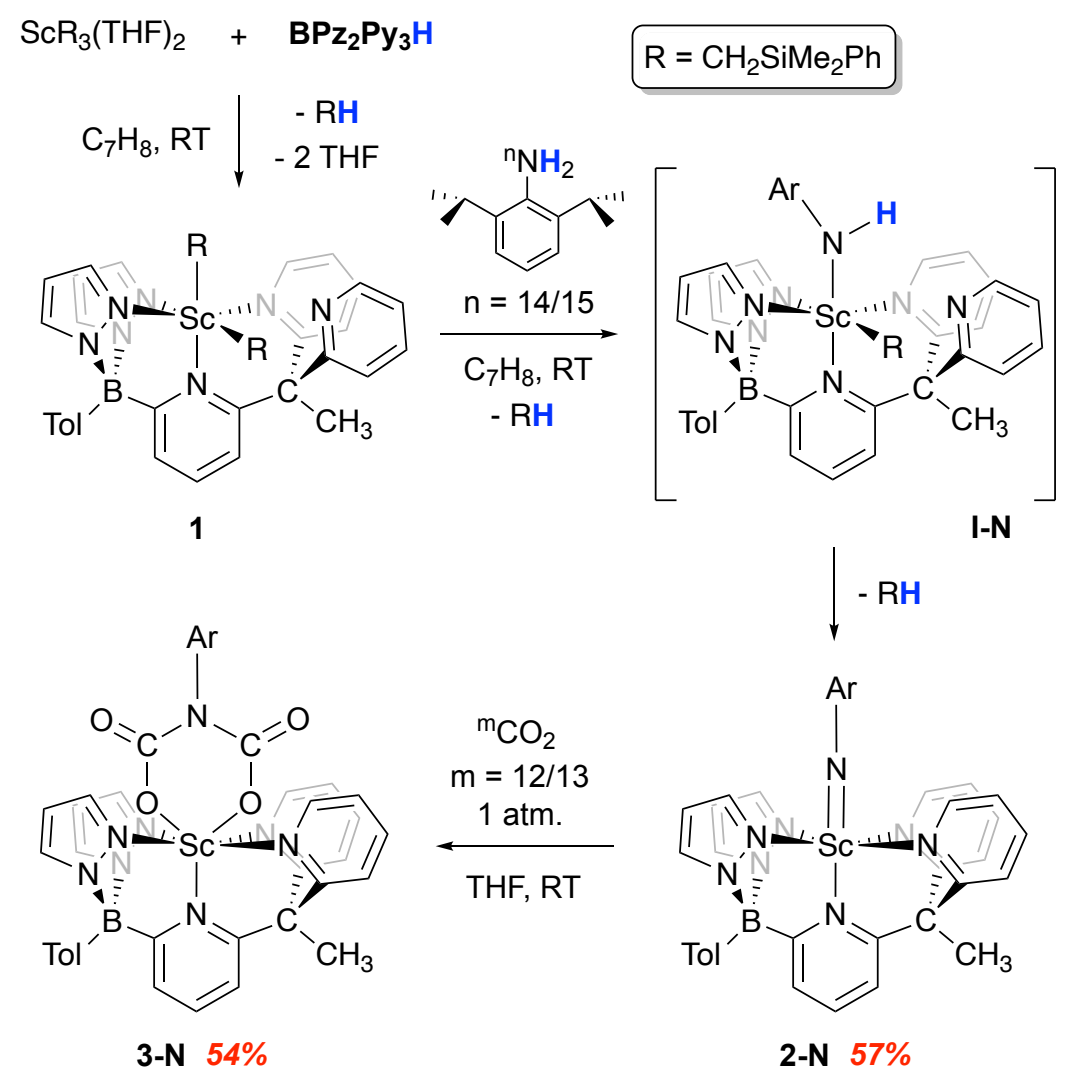

Scheme 1. Synthesis of scandium imide 2-N and its reaction with $\mathrm{CO}_{2}$. 
complex 1 (Scheme 1). Attempts to isolate this material were not successful due to its thermal instability, which may involve ortho sigma bond metathesis with the dangling pyridyl donor. ${ }^{24}$ The room temperature ${ }^{1} \mathrm{H}$ NMR spectrum of $\mathbf{1}$ is complex and broadened, indicating fluxionality that involves the exchange of bound and dangling pyridyl ligand arms. A variable temperature study supports this hypothesis, but full characterization of this material was not possible and typically it was generated in situ and used immediately without purification.

Bis-alkyl 1 reacts cleanly with 2,6-di-iso-propylphenyl aniline (with either ${ }^{14} \mathrm{~N}$ or enriched in ${ }^{15} \mathrm{~N}$ ) in a sequential double alkane elimination sequence as shown in Scheme 1 . The first alkane elimination is more rapid than the second and complete conversion to the terminal scandium imido complex $\mathbf{2}-\mathbf{N}$ requires a few days of stirring at room temperature. The compound was isolated as a purple precipitate in $57 \%$ yield and was fully characterized by multinuclear NMR spectroscopy, IR and UV-vis spectroscopies, X-ray crystallography and Density Functional Theory (DFT) computations. In the ${ }^{1} \mathrm{H}$ NMR spectrum, resonances for 15 inequivalent aromatic and pyrazolyl hydrogens are observed, ranging from a diagnostic downfield doublet at $9.88 \mathrm{ppm}$ due to the protons alpha to the equatorial pyridyl donors to a triplet at $5.83 \mathrm{ppm}$ for the single para proton on the imido aryl group. A relatively sharp signal at $291.8 \mathrm{ppm}$ (width at half height $\approx 16 \mathrm{~Hz}$ ) was detected in the ${ }^{15} \mathrm{~N} N \mathrm{NMR}$ spectrum of $2-15 \mathrm{~N}$ (Figure S19), and a band at $920 \mathrm{~cm}^{-1}$ in the IR spectrum that shifts to $911 \mathrm{~cm}^{-1}$ in the labelled isotopologue can be ascribed to the $\mathrm{Sc}=\mathrm{N}$ stretch. Crystals grown from THF/toluene revealed the molecular structure shown in Figure 1A. The Sc-N8 distance of 1.877(3) $\AA$ is consistent with comparable bonds (Chart 1) and indicative of double bond character, as is the nearly linear Sc-N8-Cipso angle of 173.1(3) ${ }^{\circ}$. DFT computations indicated a Wiberg Bond Index of 1.298 for this bond, and the HOMO and HOMO-1 orbitals show clear $\pi$ bonding between Sc and N (Figure 1B). Some delocalization of charge from the imide $N$ into the 2,6-di-iso-propylaryl ring is indicated by the rather short N-C distance of $1.36 \AA$ and the WBI of 1.219 for this bond. The Sc-N5 distance of 2.371(3) A is elongated in comparison to the other, 
equatorial pyridyl donors due to the strongly trans influencing imido donor but this not well reproduced by the computations due to the dative nature of this bond. The intense colour of the compound $\left(\lambda_{\max }=532 \mathrm{~nm}, \varepsilon=1200 \mathrm{~L} \mathrm{~mol}^{-1} \mathrm{~cm}^{-1}\right)$ derives from an absorption involving the more metal-based HOMO/HOMO-1 orbitals to a ligandbased LUMO associated with the tri-pyridyl donor locus.
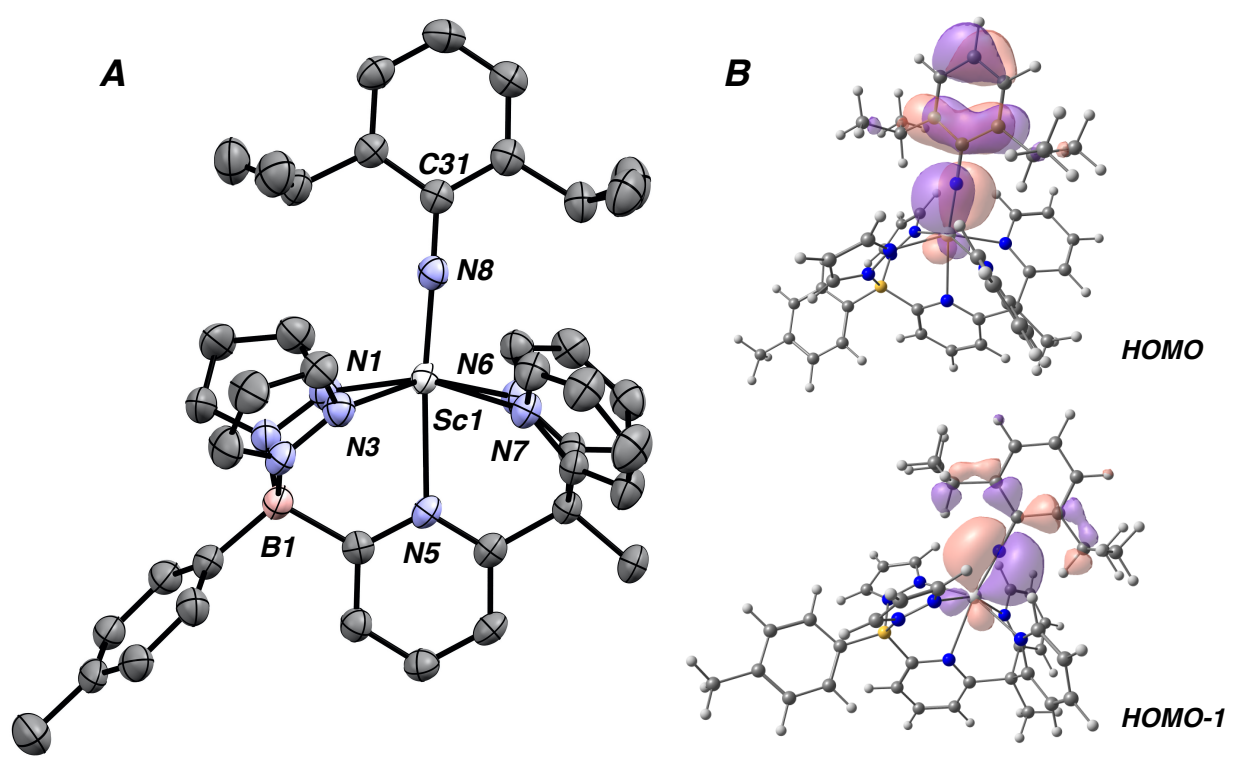

Figure 1. (A) Molecular structures of 2-N. Hydrogen atoms have been omitted for clarity. Thermal ellipsoids are shown at the $50 \%$ probability level. Selected bond lengths ( $\AA$ ) and angles $\left({ }^{\circ}\right)$ : Sc1-N1, 2.278(3); Sc1-N3, 2.268(3); Sc1-N5, 2.371(3); Sc1-N6, 2.285(3); Sc1-N7, 2.272(3); Sc1-N8, 1.877(3); N8-C31, 1.350(4); N1-Sc1N3, 84.4(1); N3-Sc1-N7, 94.1(1); N7-Sc1-N6, 78.1(1); N6-Sc1-N1, 95.4(1); Sc1N8-C31, 173.1(3). (B) DFT computed HOMO and HOMO-1 orbitals of 2-N depicting the Sc-N $\pi$ bonds.

While the imide nitrogen bears significant negative charge (basicity), 2-N shows good thermal stability in solution and does not react under ambient conditions with $\mathrm{H}_{2}{ }^{25}$ However, rapid uptake of two equivalents of $\mathrm{CO}_{2}$ is observed and 2-N undergoes clean conversion to a single complex tentatively assigned as $\mathbf{3 - N}$ (Scheme 1). This assertion is based on literature precedent ${ }^{7}$ and the coupling patterns found in the ${ }^{15} \mathrm{~N}\left(136.9 \mathrm{ppm}\right.$, triplet, $\left.{ }^{1} \mathrm{~J}_{\mathrm{N}-\mathrm{C}}=17.3 \mathrm{~Hz}\right)$ and ${ }^{13} \mathrm{C}\left(154.0 \mathrm{ppm}\right.$, doublet, ${ }^{1} \mathrm{~J}_{\mathrm{C}-\mathrm{N}}=$ 
17.4 Hz) NMR spectra of the labelled $\mathbf{3}^{-15} \mathbf{N}-\left({ }^{13} \mathbf{C}\right)_{2}$ isotopologue. Two bands in the IR spectrum $\left(1632,1685 \mathrm{~cm}^{-1}\right)$ that shift in the labelled compound are assigned to symmetric and antisymmetric $\mathrm{C}=0$ stretches. Previous work has shown that scandium complexes of dianionic ligand $\mathbf{B}_{2} \mathbf{P} \mathbf{z}_{4} \mathbf{P y}$ can accommodate $\kappa^{2}$ carboxylate $^{17}$ or $\mathrm{NTf}_{2}{ }^{18}$ ligands, so it is likely the dicarboxylate ligand in $\mathbf{3}-\mathbf{N}$ adopts this bonding motif.

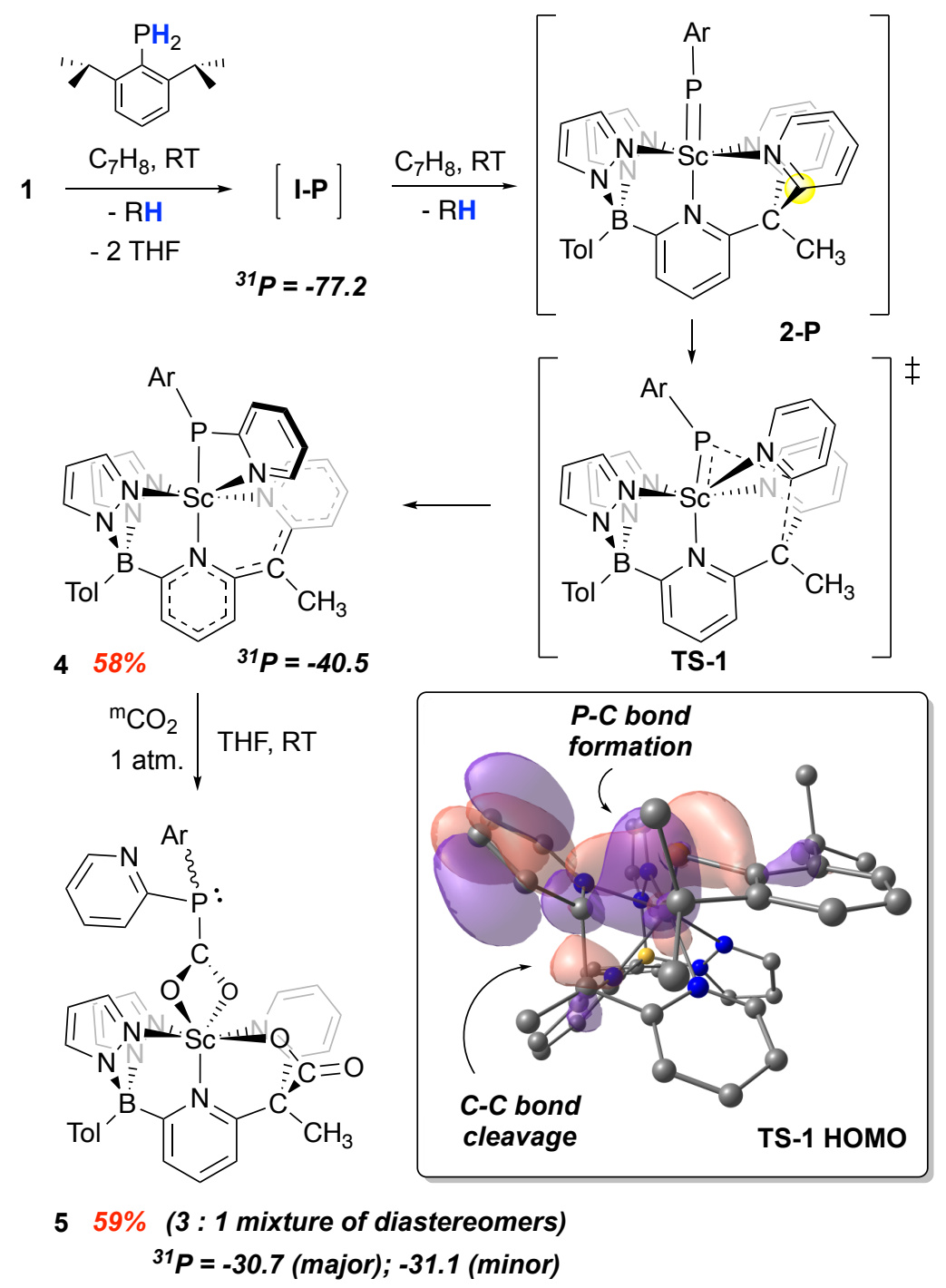

Scheme 2. Reaction of $\mathbf{1}$ with $\mathrm{ArPH}_{2}$ and $\mathrm{C}-\mathrm{C}$ bond cleavage by an intermediate phosphinidene. Reaction of product 4 with $\mathrm{CO}_{2}$. Inset: $\mathrm{HOMO}$ of the transition state for C-C bond cleavage as computed by DFT. 
The reaction of the dialkyl complex 1 with 2,6-di-iso-propylphenyl phosphine ${ }^{26}$ was undertaken to prepare the phosphinidene analog 2-P; the outcome of this reaction was not directly comparable to the aniline chemistry, forming the unanticipated product 4 (Scheme 2). When monitored by proton coupled ${ }^{31}$ P NMR spectroscopy, the reaction looked promising in that the triplet for the starting phosphine gradually disappeared as alkane was eliminated, generating a product with a singlet ${ }^{31} \mathrm{P}$ resonance at $-40.5 \mathrm{ppm}$. Also present in this reaction, at an essentially steady state, is a species characterized by a doublet $\left({ }^{1} \mathrm{JP}_{\mathrm{P} H}=221 \mathrm{~Hz}\right)$ at $-77.2 \mathrm{ppm}$, assigned as the phosphido alkyl intermediate I-P, analogous to I-N; upon reaction completion, only the signal for 4 remains. DOSY NMR spectroscopy, using 2-N and some monomeric and dimeric scandium complexes of the $\mathbf{B}_{2} \mathbf{P} \mathbf{z}_{4} \mathbf{P y}$ ligand ${ }^{17}$ as comparators, supported a monomeric formulation for 4 (Figures S3/S4 and Table S1). However, although experimental values are scarce, the upfield ${ }^{31} \mathrm{P}$ chemical shift is not indicative of a $\mathrm{Sc}=\mathrm{P}$ moiety and the proton and ${ }^{13} \mathrm{C}$ NMR data imply a lower symmetry structure than predicted for the phosphinidene 2-P. X-ray crystallographic analysis of this material (Figure $2 \mathrm{~A}$ ) revealed its structure to be that of the depicted product.

Product $\mathbf{4}$ features a novel dianionic tetradentate ligand with pyrazolyl borate and dipyridyl methide components and a $\kappa^{2}$ phosphidopyridyl ligand derived from the transfer of a pyridyl group from the $\mathbf{B P z} \mathbf{z}_{2} \mathbf{P y} \mathbf{y}_{3}$ ligand to phosphorus as a result of an unusual C-C bond cleavage step (Scheme 2). The formation of $\mathbf{4}$ can be described as a direct nucleophilic attack on the secondary ortho carbon (highlighted in yellow, Scheme 2) of a coordinated pyridyl by the basic phosphinidene phosphorus in 2-P; this $\mathrm{P}-\mathrm{C}$ bond forming step is concomitant with the cleavage of a ligand $\mathrm{C}-\mathrm{C}$ bond to generate the product. This path is supported by DFT computations in which TS-1 was located on the potential energy surface (PES) $25.6 \mathrm{kcal} \mathrm{mol}^{-1}$ above 2-P; in toluene, the barrier was calculated to be $19.8 \mathrm{kcal} \mathrm{mol}^{-1}$, in line with a kinetically facile reaction. At the TS, the Sc-P bond is elongated by $0.25 \AA$ and the phosphinidene ligand is strongly bent at the phosphorus (Sc-P-C angle of $114^{\circ} ; c f$. 
the value of $169^{\circ}$ computed for $2-\mathrm{P}$ ). This is indicative of the disruption of the Sc-P $\pi$ bond to relocalize a lone pair at the phosphorus as highlighted by the Sc-P WBI of 0.95 compared to 1.97 in 2-P. This lone pair can thus interact with an empty $\pi^{*}$ orbital of the pyridyl ligand (see the HOMO in the inset of Scheme 2). The associated P-C distance is $2.33 \AA$ and simultaneously the C-C bond distance in the pyrazolyl borate increases to $1.68 \AA$, indicating a strong activation of this bond which is highlighted by the fact that the HOMO at the TS displays an antibonding C-C interaction. Following the intrinsic reaction coordinate leads directly to the final product 4, whose formation is strongly exothermic $\left(-49.9 \mathrm{kcal} \mathrm{mol}^{-1}\right)$. Notably, the same transformation involving imido complex $\mathbf{2}-\mathbf{N}$ has a significantly higher kinetic barrier (44.8 $\left.\mathrm{kcal} \mathrm{mol}^{-1}\right)$ consistent with its observed isolability.
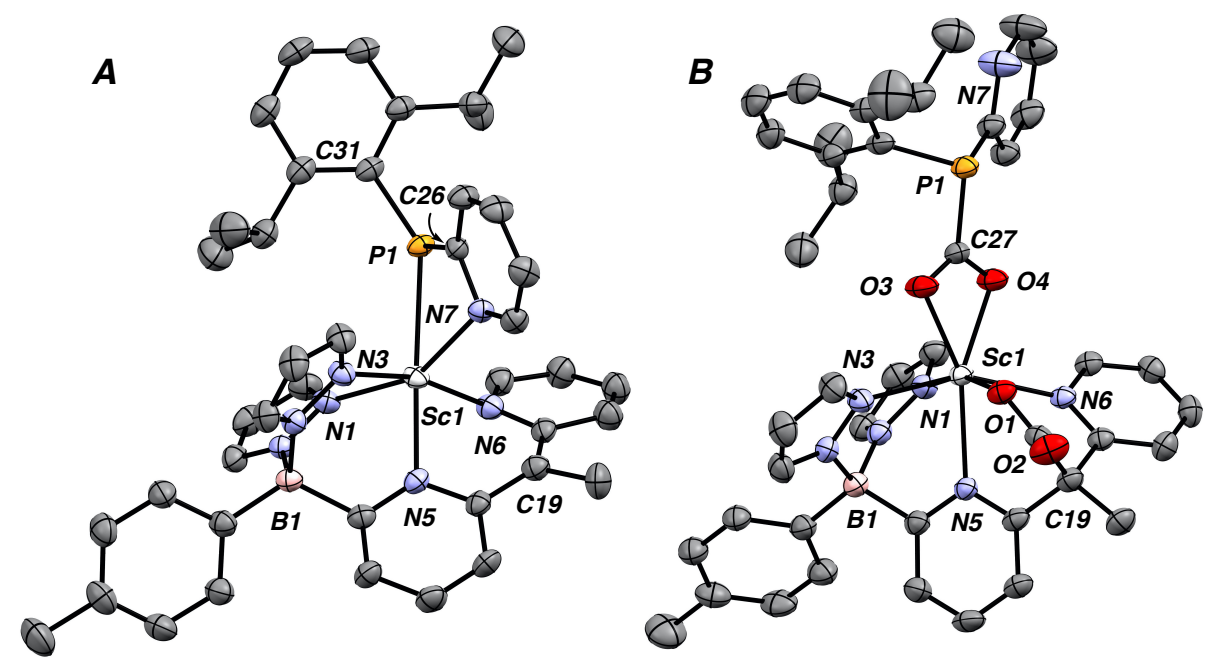

Figure 2. Molecular structures of 4 (A) and 5 (B). Hydrogen atoms have been omitted for clarity. Given the disordered diastereomeric nature of $\mathbf{5}$, the minor component is not depicted. Thermal ellipsoids are shown at the $50 \%$ probability level. Selected bond lengths ( $\AA$ ) and angles ( ${ }^{\circ}$ ) for 4: Sc1-N1, 2.207(1); Sc1-N3, 2.263(1); Sc1-N5, 2.156(1); Sc1-N6, 2.15(1); Sc1-N7, 2.210(1); Sc1-P1, 2.7529(6); P1-C31, 1.852(2); N1-Sc1-N3, 82.24(5); N3-Sc1-N7, 88.17(5); N7-Sc1-N6, 96.5(3); N6-Sc1-N1, 98.9(3); Sc1-P1-C31, 140.56(5); Sc1-P1-C26, 74.69(5); P1-C26-N7, 111.5(1). Selected bond lengths $(\AA)$ and angles $\left({ }^{\circ}\right)$ for $\mathbf{5}$ (R/R diastereomer shown): Sc1-N1, 2.207(3); Sc1-N3, 2.217(3); Sc1-N5, 2.381(2); Sc1-N6, 2.295(2); Sc1-01, 
2.012(2); Sc1-03, 2.234(2); Sc1-04, 2.198(2); C27-03, 1.258(3); C27-04, 1.262(3);

C27-P1, 1.851(2); N1-Sc1-N3, 81.50(9); N3-Sc1-01, 99.24(9); 01-Sc1-N6, 81.07(8); N6-Sc1-N1, 90.74(8); Sc1-03-C27, 90.0(2); Sc1-04-C27, 91.6(2); O3C27-04, 119.5(2).

The molecular structure of 4 (Figure 2A) shows a distorted octahedral geometry by virtue of the strained 4-membered ring in the $\mathrm{k}^{2}$-phosphidopyridyl ligand, which features a relatively long Sc-P1 bond of 2.7529(6) $\AA$ ( $c f$. the distances of 2.564(1) $\AA^{13}$ and $2.547(1) \AA^{27}$ in recently reported comparative complexes). The Sc-N5 and Sc-N6 distances of 2.156(1) and 2.15(1) Å are shortened significantly in the newly anionic dipyridyl methide fragment in comparison to those found in 2-N.

Prior to ascertaining the true identity of $\mathbf{4}$, it was observed to react rapidly with an excess of $\mathrm{CO}_{2}$, again taking up two equivalents to form the phosphinocarboxylato product 5 as a mixture of two diasteriomers in a $3: 1$ ratio. One equivalent of $\mathrm{CO}_{2}$ inserts into the Sc-P bond, ${ }^{28}$ while a second adds across the dipyridylmethide ligand in a similar fashion to what has been observed in scandium $\beta$-diketiminato complexes. ${ }^{29}$ In each process, a new chiral centre (one at $\mathrm{P}$ and one at the dipyridylmethide carbon) is generated, and thus two enantiomeric pairs of diastereomers obtain. The ${ }^{1} \mathrm{H}$ NMR spectra that result are complex, but signals in the ${ }^{31} \mathrm{P}\left\{{ }^{1} \mathrm{H}\right\}$ NMR spectra at -30.7 (major) and -31.1 (minor) ppm indicate these are the only two $\mathrm{P}$ containing species present. When ${ }^{13} \mathrm{CO}_{2}$ is employed, these signals split into doublets ( ${ }^{1} \mathrm{JPC}=15.5$ and $16.9 \mathrm{~Hz}$, respectively) and analogous doublets appear at 199.09 and $198.64 \mathrm{ppm}$ in the ${ }^{13} \mathrm{C}\left\{{ }^{1} \mathrm{H}\right\}$ NMR spectrum. The other carboxylate gives rise to singlets at 169.88 and $169.82 \mathrm{ppm}$ for the major and minor diastereomers, respectively. The structure was confirmed by X-ray crystallography (Figures 2B and S4/S6). During the structural refinement, significant residual electron density remained around the phosphine phosphorus and modelling this led to the conclusion that it was due to the presence of the other diastereomer in the 
lattice. Despite this disorder, the metrical parameters are well defined and nothing unusual is noted in the bond lengths and angles.

In conclusion, we have designed and synthesized a new pentadentate ligand platform for supporting group 3 metal element double bonds and demonstrate its efficacy for scandium imido and transient phosphinidene compounds. The high basicity of the pnictogen atom is evident in the former's reactivity with $\mathrm{CO}_{2}$ and the latter's propensity to nucleophilically attack a pyridyl arm of the ligand. Use of this ligand for a range of group 3/lanthanide and other first row metal complexes is under current investigation.

\section{Acknowledgements}

Funding for this work was provided by NSERC of Canada in the form of a Discovery Grant to W.E.P. who also acknowledges the Canada Research Chair secretariat for a Tier I CRC (2020-2027). E.A.P thanks Alberta Innovates for scholarship support. The computational work was supported by the HPCs CALcul en Midi-Pyrénées (CALMIP-EOS grant 1415); L. M. is a senior member of the Institut Universitaire de France. L.M and Y.Y also thank the Humboldt Foundation, the Chinese Academy of Sciences and the Chinese Scholarship Council.

\section{Conflicts of interest}

There are no conflicts to declare.

\section{References}

1. G. R. Giesbrecht and J. C. Gordon, Dalton Trans., 2004, DOI: 10.1039/B407173E, 2387-2393.

2. $\quad$ O. T. Summerscales and J. C. Gordon, RSC Adv., 2013, 3, 6682-6692.

3. D. Schädle, M. Meermann-Zimmermann, C. Schädle, C. Maichle-Mössmer and R. Anwander, Eur. J. Inorg. Chem., 2015, 2015, 1334-1339. 
4. D. Schädle and R. Anwander, Chem. Soc. Rev., 2019, 48, 5752-5805.

5. E. Lu, J. Chu and Y. Chen, Acc. Chem. Res., 2018, 51, 557-566.

6. E. Lu, Y. Li and Y. Chen, Chem. Comm., 2010, 46, 4469-4471.

7. J. Chu, E. Lu, Z. Liu, Y. Chen, X. Leng and H. Song, Angew. Chem. Int. Ed., 2011, 50, 7677-7680.

8. W. Rong, J. Cheng, Z. Mou, H. Xie and D. Cui, Organometallics, 2013, 32, 55235529.

9. D. J. Beetstra, A. Meetsma, B. Hessen and J. H. Teuben, Organometallics, 2003, 22, 4372-4374.

10. J. Scott, F. Basuli, A. R. Fout, J. C. Huffman and D. J. Mindiola, Angew. Chem. Int. Ed., 2008, 47, 8502-8505.

11. J. Chu, X. Han, C. E. Kefalidis, J. Zhou, L. Maron, X. Leng and Y. Chen, J. Am. Chem. Soc., 2014, 136, 10894-10897.

12. B. F. Wicker, J. Scott, J. G. Andino, X. Gao, H. Park, M. Pink and D. J. Mindiola, J. Am. Chem. Soc., 2010, 132, 3691-3693.

13. B. Feng, L. Xiang, A. Carpentier, L. Maron, X. Leng and Y. Chen, J. Am. Chem. Soc., 2021, 143, 2705-2709.

14. D. M. Spasyuk, S. H. Carpenter, C. E. Kefalidis, W. E. Piers, M. L. Neidig and L. Maron, Chem. Sci., 2016, 7, 5939-5944.

15. L. Nurdin, D. M. Spasyuk, W. E. Piers and L. Maron, Inorg. Chem., 2017, 56, 4157-4168.

16. L. Nurdin, D. M. Spasyuk, L. Fairburn, W. E. Piers and L. Maron, J. Am. Chem. Soc., 2018, 140, 16094-16105.

17. D. W. Beh, W. E. Piers, I. del Rosal, L. Maron, B. S. Gelfand, C. Gendy and J.-B. Lin, Dalton Trans., 2018, 47, 13680-13688.

18. D. W. Beh, W. E. Piers, L. Maron, Y. Yang, B. S. Gelfand and J.-B. Li, Polyhedron, 2020, 179, 114410.

19. D. W. Beh, W. E. Piers, B. S. Gelfand and J.-B. Lin, Dalton Trans., 2020, 49, 95101.

20. D. W. Beh, Ph. D., University of Calgary, 2019.

21. R. T. Jonas and T. D. P. Stack, J. Am. Chem. Soc., 1997, 119, 8566-8567.

22. M. E. de Vries, R. M. La Crois, G. Roelfes, H. Kooijman, A. L. Spek, R. Hage and B. L. Feringa, Chem. Comm., 1997, DOI: 10.1039/A702804K, 1549-1550.

23. D. J. H. Emslie, W. E. Piers, M. Parvez and R. McDonald, Organometallics, 2002, 21, 4226-4240.

24. M. E. Thompson, S. M. Baxter, A. R. Bulls, B. J. Burger, M. C. Nolan, B. D. Santarsiero, W. P. Schaefer and J. E. Bercaw, J. Am. Chem. Soc., 1987, 109, $203-$ 219.

25. X. Han, L. Xiang, C. A. Lamsfus, W. Mao, E. Lu, L. Maron, X. Leng and Y. Chen, Chem. Eur. J., 2017, 23, 14728-14732.

26. R. T. Boeré and J. D. Masuda, Can. J. Chem., 2002, 80, 1607-1617.

27. B. Feng, L. Xiang, K. N. McCabe, L. Maron, X. Leng and Y. Chen, Nat. Commun., 2020, 11, 2916.

28. G. A. Vaughan, G. L. Hillhouse and A. L. Rheingold, Organometallics, 1989, 8, 1760-1765. 
29. F. A. LeBlanc, A. Berkefeld, W. E. Piers and M. Parvez, Organometallics, 2012, 31, 810-818.

30. A. B. Pangborn, M. A. Giardello, R. H. Grubbs, R. K. Rosen and F. J. Timmers, Organometallics, 1996, 15, 1518-1520.

31. L. Doszczak, P. Kraft, H.-P. Weber, R. Bertermann, A. Triller, H. Hatt and R. Tacke, Angewandte Chemie International Edition, 2007, 46, 3367-3371.

32. E. A. Ünal, D. Wiedemann, J. Seiffert, J. P. Boyd and A. Grohmann, Tetrahedron Letters, 2012, 53, 54-55.

33. I. Saeed, S. Katao and K. Nomura, Inorganic Chemistry, 2009, 48, 5011-5020.

34. R. A. Green and J. F. Hartwig, Organic Letters, 2014, 16, 4388-4391.

35. G. R. Fulmer, A. J. M. Miller, N. H. Sherden, H. E. Gottlieb, A. Nudelman, B. M. Stoltz, J. E. Bercaw and K. I. Goldberg, Organometallics, 2010, 29, 2176-2179.

36. Bruker-AXS. SAINT. Madison, Wisconsin, USA, 2017.

37. Bruker-AXS. XPREP. Madison, Wisconsin, USA, 2017.

38. O. V. Dolomanov, L. J. Bourhis, R. J. Gildea, J. A. K. Howard and H. Puschmann, Journal of Applied Crystallography, 2009, 42, 339-341.

39. G. M. Sheldrick, Acta Crystallographica a-Foundation and Advances, 2015, 71, 3-8.

40. G. M. Sheldrick, Acta Crystallographica Section C-Structural Chemistry, 2015, 71, 3-8.

41. A. L. Spek, Acta Crystallographica Section C-Structural Chemistry, 2015, 71, 918.

42. J. H. Barnard, S. Yruegas, S. A. Couchman, D. J. D. Wilson, J. L. Dutton and C. D. Martin, Organometallics, 2016, 35, 929-931.

43. G. A. Morris, in eMagRes, DOI: https://doi.org/10.1002/9780470034590.emrstm0119.pub2.

44. S. Matsuo and T. Makita, International Journal of Thermophysics, 1993, 14, 6777.

45. B. M. Schulze, D. L. Watkins, J. Zhang, I. Ghiviriga and R. K. Castellano, Organic \& Biomolecular Chemistry, 2014, 12, 7932-7936. 


\section{Supporting Information}

\section{A Monoanionic Pentadentate Ligand Platform for Scandium-Pnictogen Multiple Bonds}

Evan A. Patrick, ${ }^{a}$ Yan Yang, ${ }^{b}$ Warren E. Piers, ${ }^{a *}$ Laurent Maron, ${ }^{b *}$ and Benjamin S. Gelfand ${ }^{a}$

aUniversity of Calgary, Department of Chemistry, 2500 University Drive N.W., Calgary, Alberta, Canada, T2N $1 \mathrm{~N} 4$.

'LPCNO, Université de Toulouse, INSA, UPS, LPCNO, 135 avenue de Rangueil, F-31077 Toulouse, France, and CNRS, LPCNO, F-31077 Toulouse, France

\section{$\underline{\text { Table of Contents }}$}

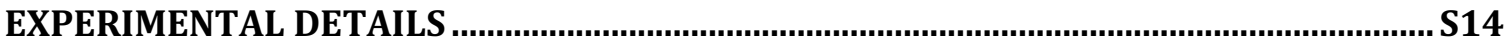

GENERAL CONSIDERATIONS

EXPERIMENTAL PROCEDURE

Dichlorotolylborane:

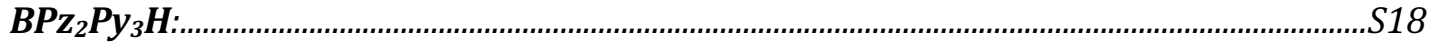

2-N:

3-N:

$S 20$

4:.

S23

5

SUPPORTING FIGURES

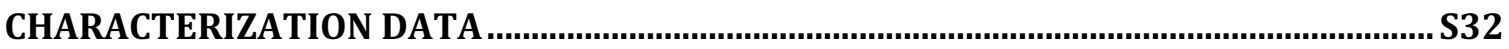

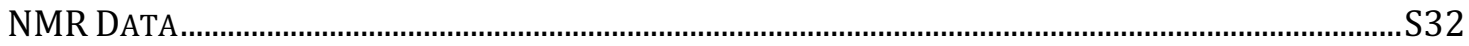

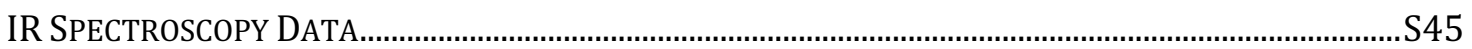

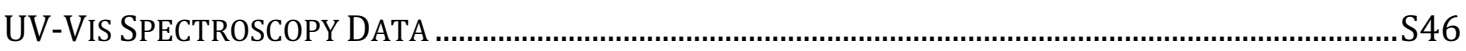

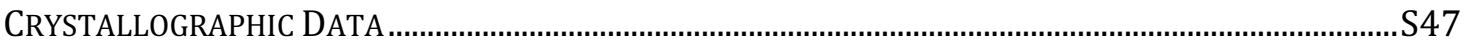

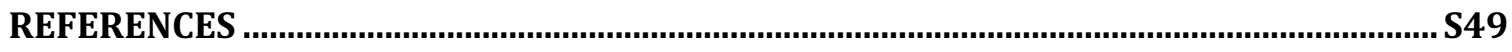

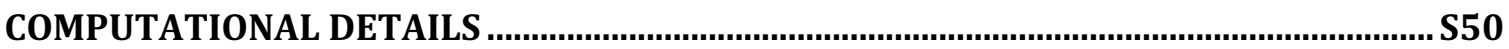

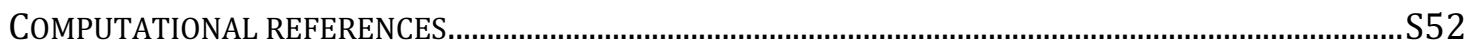

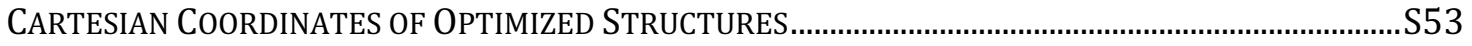




\section{Experimental Details}

\section{General Considerations}

Manipulation and storage of all air/moisture sensitive materials was performed under an argon atmosphere in an MBRAUN glove box. Reactions were performed on a double manifold high vacuum line fitted with an OxisorBW scrubber (Matheson Gas products) argon purification cartridge, using standard techniques. Glassware was stored at $135^{\circ} \mathrm{C}$ in an oven overnight prior to immediate transfer to the glovebox antechamber or assembly on the vacuum line and evacuated while hot.

Toluene, THF and $n$-pentane were dried and purified using a Grubbs/Down purification system, ${ }^{30}$ and stored in evacuated thick-walled vessels over sodium/benzophenone ketal. Benzene, benzene- $d_{6}$, toluene- $d_{8}$, and THF- $d_{8}$ were dried and stored over sodium/benzophenone ketyl. 1,2-dichlorobenzene, 1,2dichlorobenzene- $d_{4}$, dichloromethane, and $\mathrm{CD}_{2} \mathrm{Cl}_{2}$ were dried over $\mathrm{CaH}_{2}$. All dried solvents were degassed and vacuum transferred prior to use into thick-walled glass vessels for storage.

4-(trimethylsilyl)toluene, ${ }^{31} \quad$ 2,2'-[1-(6-Bromopyridin-2-yl)ethane-1,1diyl]dipyridine, ${ }^{32}$ lithium pyrazolate, ${ }^{33} \quad \mathrm{Sc}\left(\mathrm{CH}_{2} \mathrm{SiMe}_{2} \mathrm{Ph}\right)_{3}(\text { thf })_{2},{ }^{23} \quad$ 2,6diisopropylaniline-15 $\mathrm{N},{ }^{34}$ and 2,6-diisopropylphosphine ${ }^{26}$ were prepared according to literature procedures. Pyrazole was sublimed, and 2,6-diisopropylaniline was dried over $\mathrm{CaH}_{2}$ and distilled under reduced pressure prior to use. All other chemicals were obtained from commercial suppliers and used without further purification. $\mathrm{CO}_{2}$ (Coleman Instrument grade, 99.99\%) was purchased from Air Liquide and used as received. ${ }^{13} \mathrm{CO}_{2}(99 \%)$ was purchased from Sigma-Aldrich and used as received.

Nuclear magnetic resonance spectroscopy experiments were performed on either an Ascend-500 or Avance-600 Bruker spectrometer. All ${ }^{1} \mathrm{H}$ and ${ }^{13} \mathrm{C}\left\{{ }^{1} \mathrm{H}\right\} \mathrm{NMR}$ 
spectra were internally referenced relative to $\mathrm{Si}(\mathrm{CH} 3)_{4}$ using residual solvent protons and naturally abundant ${ }^{13} \mathrm{C}$ resonances for all deuterated solvents ${ }^{35}$, except for 1,2-dichlorobenzene- $d_{4}$ which was referenced relative to HMDSO using residual solvent protons and naturally abundant ${ }^{13} \mathrm{C}$ resonances. ${ }^{15} \mathrm{~N}$ NMR was externally referenced to $90 \%$ nitromethane in $\mathrm{CDCl}_{3}$. NMR spectra were processed and analyzed with MestReNova (v. 9.0.1- 13254).

X-ray crystallographic analyses were performed by Dr. Benjamin Gelfand, and structural solutions generated by both Dr. Gelfand and Evan Patrick. Single crystals of each complex were grown as described in the experimental procedure section. Crystals were coated in Fomblin Y HVAC 140/13 oil, and a suitable crystal was selected and mounted on a glass loop. Diffraction experiments were performed on a Bruker Smart diffractometer using either a Incoatec Microfocus $(\mathrm{Cu} \mathrm{Ka}, \lambda=1.54178 \AA)$ or Siemens Fine Focus Ceramic Tube (graphite monochromated Mo Ka, $\lambda=0.71069 \AA$ ) ), and an APEX II CCD detector. The crystal was kept at $173 \mathrm{~K}$ during data collection. Diffraction spots were integrated and scaled with SAINT ${ }^{36}$ and the space group was determined with XPREP. ${ }^{37}$ Using Olex2,38 the structure was solved with the ShelXT'39 structure solution program using Intrinsic Phasing and refined with the SheIXL ${ }^{40}$ refinement package using Least Squares minimization. In order to improve the completeness of the reflections collected for 4 , the crystal was remounted to a new position, missing reflections collected, and the resulting datasets were merged isotropically with XPREP. ${ }^{37}$ Electron density contributions from non-coordinating solvent molecules in $\mathbf{2}-\mathbf{N}$ were modelled using the SQUEEZE routine in PLATON. ${ }^{41}$ More details on individual structures can be found in Table S2.

Elemental analyses were performed by Johnson Li using a Elementar UNICUBE analyzer at the Instrumentation Facility of the Department of Chemistry, University of Calgary. Infrared spectra were also collected by Mr. Li on a Nicolet Avater FT-IR spectrometer with samples prepared as $\mathrm{KBr}$ pellets. Solution highresolution mass spectrometry (ESI-MS) was performed by Wade White using a Kratos MS-80 spectrometer on samples prepared in a glovebox and 
transported/injected via gas-tight syringe. Absorption spectra were measured using a Varian Cary-50 single beam spectrophotometer. 


\section{Experimental Procedure}

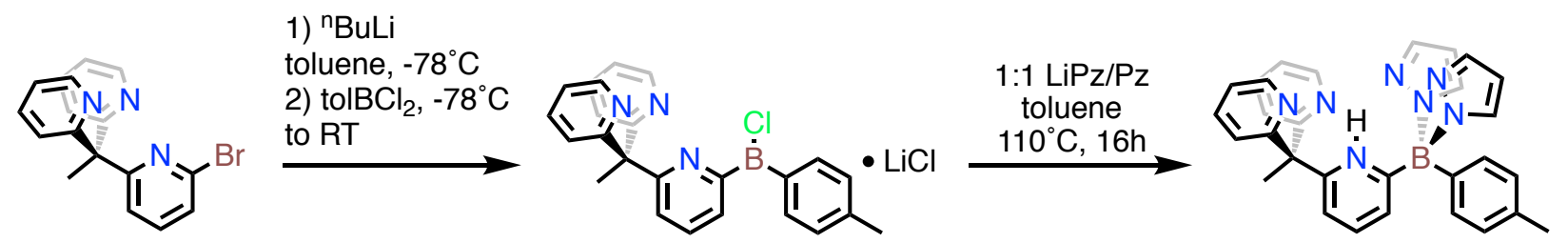

Scheme $\mathrm{S1}$ - Overview of $\mathrm{BPz}_{2} P y_{3} \mathrm{H}$ ligand synthesis

\section{Dichlorotolylborane:}

This is a modification of a published procedure for a similar dichloroarylborane. ${ }^{42}$ A $50 \mathrm{ml}$ thick-walled glass vessel equipped with a Kontes PTFE tap and stirbar was cooled to $-78^{\circ} \mathrm{C}$ and charged with $\mathrm{BCl}_{3}(1 \mathrm{M}$ in DCM, $27 \mathrm{ml}, 26.78 \mathrm{mmol}$ ) via syringe under gentle flow of argon. A solution of 4(trimethylsilyl)toluene $(3.78 \mathrm{~g}, 24.34 \mathrm{mmol})$ in DCM $(\sim 3-5 \mathrm{ml})$ was then added dropwise via syringe. The vessel was then sealed, slowly warmed to $0^{\circ} \mathrm{C}$, and stirred for 1 hour. The reaction mixture was then allowed to warm to room temperature and stirred overnight. The vessel was then cooled to $0^{\circ} \mathrm{C}$, and the volatiles were carefully removed in vacuo (since the product is also volatile). To isolate $>95 \%$ pure material, vacuum was applied at $0^{\circ} \mathrm{C}$ for $3-4$ hours, checking progress by NMR. Product was obtained as a yellowish oil that solidifies upon cooling $(3.64 \mathrm{~g}, 92 \%)$ and stored at $-30^{\circ} \mathrm{C}$. Though dichlorotolylborane is known in the literature, no NMR data could be found.

${ }^{1} \mathrm{H}$ NMR $\left(500 \mathrm{MHz}\right.$, Benzene- $\left.d_{6}\right) \delta 7.96\left(\mathrm{~d}, J=7.8 \mathrm{~Hz}, 2 \mathrm{H}_{\mathrm{a}}\right), 6.85(\mathrm{~d}, J=7.7 \mathrm{~Hz}$, $\left.2 \mathrm{H}_{\mathrm{b}}\right), 1.93\left(\mathrm{~s}, 3 \mathrm{H}_{\mathrm{c}}\right)$.

${ }^{11} \mathrm{~B}\left\{{ }^{1} \mathrm{H}\right\}$ NMR $\left(161 \mathrm{MHz}, \mathrm{C}_{6} \mathrm{D} 6\right) \delta 55.54$.

${ }^{13} \mathrm{C}\left\{{ }^{1} \mathrm{H}\right\}$ NMR $\left(126 \mathrm{MHz}\right.$, Benzene- $\left.d_{6}\right) \delta 146.46\left(\mathrm{C}_{1}\right), 137.47\left(\mathrm{C}_{2}\right), 129.17\left(\mathrm{C}_{3}\right), 21.71$ $\left(\mathrm{C}_{4}\right)$, B-C $\mathrm{C}_{\text {ipso }}$ was not observed. 


\section{$\mathrm{BPz} \mathrm{z}_{2} \mathrm{H}$ :}

Part A:

A 2-neck 500ml RBF was charged with a stirbar, 2,2'-[1-(6-Bromopyridin-2-yl)ethane-1,1-diyl]dipyridine $(5.480 \mathrm{~g}, 16.1 \mathrm{mmol})$, and toluene $(150 \mathrm{ml})$, and cooled to

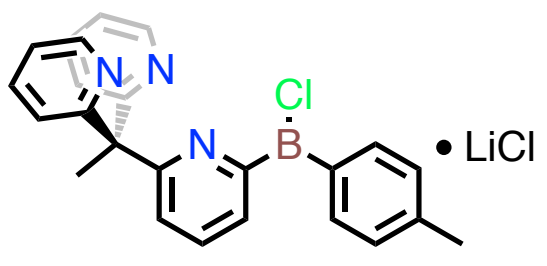
$-78^{\circ} \mathrm{C}$. ${ }^{\mathrm{n} B u L i}(1.6 \mathrm{M}$ in hexanes, $10.1 \mathrm{ml}, 16.2 \mathrm{mmol})$ was added dropwise via syringe turning the orange solution deep red, and stirred for $1 \mathrm{~h}$. Separately, a 2-neck $500 \mathrm{ml}$ RBF was charged with a stirbar, dichlorotolylborane (3.059g, 17.7mmol), and attached to the long end of a large diameter swivel frit. Toluene $(100 \mathrm{ml})$ was then added via vacuum transfer, and the solution cooled to $-78^{\circ} \mathrm{C}$. With both sides at $-78^{\circ} \mathrm{C}$, and while swabbing the needle with $L_{2}$, the lithiate solution was then cannula transferred onto the borane solution dropwise, turning cloudy beige/orange. Once complete, the mixture was stirred at $-78^{\circ} \mathrm{C}$ for $1 \mathrm{~h}$, slowly warmed to RT, and stirred for an additional $1 \mathrm{~h}$. The precipitate was then collected on the frit, washed with pentane $(3 \times 100 \mathrm{ml})$, and dried in vacuo. The resulting crude beige solid was then used in the next step without further purification.

\section{Part B:}

In a 500ml RBF, product from Part A $(5.738 \mathrm{~g}$, $13.0 \mathrm{mmol})$, pyrazole $(0.888 \mathrm{~g}, 13.0 \mathrm{mmol})$, lithium pyrazolate $(0.965 \mathrm{~g}, 13.0 \mathrm{mmol})$, and a stirbar were combined and

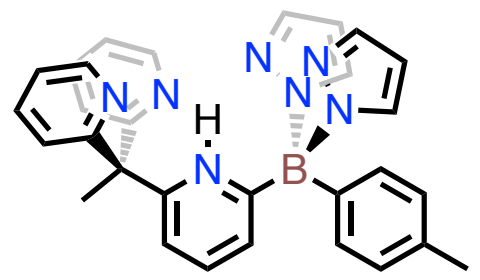
attached to the long end of a swivel frit. Toluene $(150 \mathrm{ml})$ was then vacuum transferred in, and the solution heated to $105^{\circ} \mathrm{C}$ for 12 hours. The red solution was then filtered, concentrated under vacuum $(\sim 40 \mathrm{ml})$, and set to crystallize at $-30^{\circ} \mathrm{C}$. After four days, the first crop of faint pink crystals was collected, washed with pentane, and dried in vacuo. A second crop of crystals was obtained by concentrating the toluene filtrate further $(\sim 20 \mathrm{ml})$ and cooling again to $-30^{\circ} \mathrm{C}$ (total isolated material $4.567 \mathrm{~g}, 57 \%$ over both steps). Single crystals suitable for $\mathrm{x}$-ray diffraction were grown from a concentrated toluene solution at $-30^{\circ} \mathrm{C}$. Elemental 
Analysis: Calcd. (\%) for $\mathrm{C}_{30} \mathrm{H}_{28} \mathrm{BN}_{7}$ : $\mathrm{C}, 72.44 ; \mathrm{H}, 5.67 ; \mathrm{N}, 19.71$. Found: $\mathrm{C}, 72.09$; H, 5.43; N, 19.25.

${ }^{1} \mathrm{H}$ NMR $\left(500 \mathrm{MHz}, \mathrm{CD}_{2} \mathrm{Cl}_{2}\right) \delta 17.02\left(\mathrm{~s}, 1 \mathrm{H}, \mathrm{H}_{\mathrm{a}}\right), 8.50$ (ddd, $J=4.8,1.9,0.9 \mathrm{~Hz}$, $2 \mathrm{H}, \mathrm{H}_{\mathrm{b}}$ ), 7.94 (t, $J=7.9 \mathrm{~Hz}, 1 \mathrm{H}, \mathrm{H}_{\mathrm{c}}$ ), 7.71 (td, $\left.J=7.8,1.9 \mathrm{~Hz}, 2 \mathrm{H}, \mathrm{H}_{\mathrm{d}}\right), 7.59$ (dd, $J$ $\left.=7.9,1.2 \mathrm{~Hz}, 1 \mathrm{H}, \mathrm{H}_{\mathrm{e}}\right), 7.41\left(\mathrm{~d}, J=1.6 \mathrm{~Hz}, 2 \mathrm{H}, \mathrm{H}_{\mathrm{f}}\right), 7.29$ (dd, $J=8.1,1.3 \mathrm{~Hz}, 1 \mathrm{H}$, $\mathrm{H}_{\mathrm{g}}$ ), 7.25 (ddd, $J=7.6,4.8,1.0 \mathrm{~Hz}, 2 \mathrm{H}, \mathrm{H}_{\mathrm{h}}$ ), 7.22 (dt, $J=8.0,1.1 \mathrm{~Hz}, 2 \mathrm{H}, \mathrm{H}_{\mathrm{i}}$ ), 7.07 $\left(\mathrm{d}, J=2.2 \mathrm{~Hz}, 2 \mathrm{H}, \mathrm{H}_{\mathrm{j}}\right), 7.01\left(\mathrm{~d}, J=7.6 \mathrm{~Hz}, 2 \mathrm{H}, \mathrm{H}_{\mathrm{k}}\right), 6.80\left(\mathrm{~d}, J=7.6 \mathrm{~Hz}, 2 \mathrm{H}, \mathrm{H}_{\mathrm{l}}\right)$, 6.17 (t, $J=1.9 \mathrm{~Hz}, 2 \mathrm{H}, \mathrm{H}_{\mathrm{m}}$ ), 2.39 (s, 3H, $\left.\mathrm{H}_{\mathrm{n}}\right), 2.30$ (s, 3H, $\mathrm{H}_{\mathrm{o}}$ ).

${ }^{11} \mathrm{~B}\left\{{ }^{1} \mathrm{H}\right\}$ NMR $\left(161 \mathrm{MHz}, \mathrm{CD}_{2} \mathrm{Cl}_{2}\right) \delta-1.23$.

${ }^{13} \mathrm{C}\left\{{ }^{1} \mathrm{H}\right\}$ NMR $\left(126 \mathrm{MHz}, \mathrm{CD}_{2} \mathrm{Cl}_{2}\right) \delta 174.39\left(\mathrm{br}, \mathrm{C}_{1}\right), 162.71\left(\mathrm{C}_{2}\right), 157.70\left(\mathrm{C}_{3}\right)$, 149.62( $\left.\mathrm{C}_{4}\right), 145.62\left(\mathrm{br}, \mathrm{C}_{5}\right), 141.22\left(\mathrm{C}_{6}\right), 139.61\left(\mathrm{C}_{7}\right), 137.27\left(\mathrm{C}_{8}\right), 136.04\left(\mathrm{C}_{9}\right)$, 134.56 $\left(\mathrm{C}_{10}\right)$, 133.11 $\left(\mathrm{C}_{11}\right), 129.92\left(\mathrm{C}_{12}\right), 128.36\left(\mathrm{C}_{13}\right), 123.30\left(\mathrm{C}_{14}\right), 122.86\left(\mathrm{C}_{15}\right)$, 122.79( $\left.C_{16}\right), 103.88\left(C_{17}\right), 58.43\left(C_{18}\right), 27.07\left(C_{19}\right), 21.28\left(C_{20}\right)$.

\section{2-N:}

A $50 \mathrm{ml}$ thick-walled glass vessel equipped with a Kontes PTFE tap and stirbar was charged with $\mathrm{BPz}_{2} \mathrm{Py}_{3} \mathrm{H}$ (214mg, $\quad 0.43 \mathrm{mmol}), \quad \mathrm{Sc}\left(\mathrm{CH}_{2} \mathrm{SiMe}_{2} \mathrm{Ph}\right)_{3}(\text { thf })_{2} \quad$ (274mg, $0.43 \mathrm{mmol})$, and toluene $(10 \mathrm{ml})$, and stirred until all material dissolved, turning the solution orange. A solution of 2,6diisopropylaniline $(76 \mathrm{mg}, 0.43 \mathrm{mmol})$ in toluene $(5 \mathrm{ml})$ was

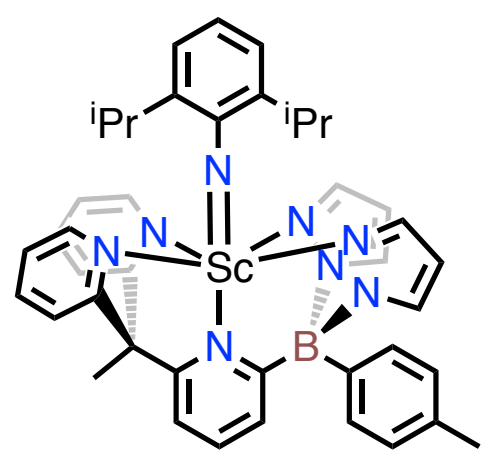
then added, and the mixture stirred for 5 days. The dark mixture was then filtered through a fine porosity frit, washed with toluene $(2 \mathrm{ml})$ and pentane $(3 \times 10 \mathrm{ml})$. The resulting purple solid $\left(175 \mathrm{mg}, 57 \%\right.$ ) was stored at $-30^{\circ} \mathrm{C}$. Similarly, the ${ }^{15} \mathrm{~N}$ labelled material $\left(2-{ }^{-15} \mathbf{N}\right)$ was synthesized using 2,6-diisopropylaniline-15N. Since this material was found to be extremely sensitive to air, moisture, as well as dichloromethane, it could only be handled in a rigorously maintained glovebox atmosphere. It was also found to be thermally sensitive as an isolated solid, and degrades over time at room temperature or upon drying completely under high 
vacuum. Single crystals suitable for $\mathrm{x}$-ray diffraction were obtained by layering toluene onto a concentrated solution of 2-N in THF. Elemental Analysis: Calcd. (\%) for 2-Notoluene $\left(\mathrm{C}_{49} \mathrm{H}_{52} \mathrm{BN}{ }_{8} \mathrm{Sc}\right)$ : $\mathrm{C}, 72.77 ; \mathrm{H}, 6.48 ; \mathrm{N}, 13.85$. Found: $\mathrm{C}, 72.45 ; \mathrm{H}$, $6.59 ; \mathrm{N}, 13.60$.

${ }^{1} \mathrm{H}$ NMR $\left(500 \mathrm{MHz}, \mathrm{THF}-\mathrm{d}_{8}\right) \delta 9.88\left(\mathrm{dd}, J=5.2,1.8 \mathrm{~Hz}, 2 \mathrm{H}, \mathrm{H}_{\mathrm{a}}\right), 8.30(\mathrm{~d}, J=2.0$ $\left.\mathrm{Hz}, 2 \mathrm{H}, \mathrm{H}_{\mathrm{b}}\right), 7.97\left(\mathrm{~d}, J=8.3 \mathrm{~Hz}, 2 \mathrm{H}, \mathrm{H}_{\mathrm{c}}\right), 7.91\left(\mathrm{td}, J=8.3,1.7 \mathrm{~Hz}, 2 \mathrm{H}, \mathrm{H}_{\mathrm{d}}\right), 7.71$ (d, $\left.J=7.9 \mathrm{~Hz}, 2 \mathrm{H}, \mathrm{H}_{\mathrm{e}}\right), 7.61\left(\mathrm{~d}, J=2.3 \mathrm{~Hz}, 2 \mathrm{H}, \mathrm{H}_{\mathrm{f}}\right), 7.58\left(\mathrm{dd}, J=8.3,1.1 \mathrm{~Hz}, 1 \mathrm{H}, \mathrm{H}_{\mathrm{g}}\right.$ ), $7.48-7.39\left(\mathrm{~m}, 3 \mathrm{H}, \mathrm{H}_{\mathrm{h}}\right), 7.25\left(\mathrm{~d}, J=7.9 \mathrm{~Hz}, 2 \mathrm{H}, \mathrm{H}_{\mathrm{i}}\right), 7.18-7.16\left(\mathrm{~m}, 1 \mathrm{H}, \mathrm{H}_{\mathrm{j}}\right), 6.41$ (d, $\left.J=7.3 \mathrm{~Hz}, 2 \mathrm{H}, \mathrm{H}_{\mathrm{k}}\right), 6.12\left(\mathrm{t}, J=2.1 \mathrm{~Hz}, 2 \mathrm{H}, \mathrm{H}_{\mathrm{I}}\right), 5.83\left(\mathrm{t}, J=7.3 \mathrm{~Hz}, 1 \mathrm{H}, \mathrm{H}_{\mathrm{m}}\right.$ ), 3.76 (hept, $J=6.8 \mathrm{~Hz}, 2 \mathrm{H}, \mathrm{H}_{\mathrm{n}}$ ), 2.74 (s, 3H, $\mathrm{H}_{\mathrm{o}}$ ), 2.41 (s, 3H, $\mathrm{H}_{\mathrm{p}}$ ), 0.62 (d, $J=6.9$ $\left.\mathrm{Hz}, 12 \mathrm{H}, \mathrm{H}_{\mathrm{q}}\right)$.

${ }^{11} \mathrm{~B}\left\{{ }^{1} \mathrm{H}\right\}$ NMR $\left(161 \mathrm{MHz}, \mathrm{THF}-\mathrm{d}_{8}\right) \delta-0.46$.

${ }^{13} \mathrm{C}\left\{{ }^{1} \mathrm{H}\right\}$ NMR (126 MHz, THF- $d_{8}$ ) $\delta 175.52$ (observed in HMBC but not in ${ }^{13} \mathrm{C}\left\{{ }^{1} \mathrm{H}\right\}$, $\left.\mathrm{C}_{26}\right), 159.73\left(\mathrm{C}_{1}\right), 158.65\left(\mathrm{C}_{2}\right), 156.63\left(\mathrm{C}_{3}\right), 152.60\left(\mathrm{C}_{4}\right), 143.83\left(\mathrm{C}_{5}\right), 143.14(\mathrm{br}$, $\left.\mathrm{C}_{6}\right), 139.89\left(\mathrm{C}_{7}\right), 139.30\left(\mathrm{C}_{8}\right), 136.74\left(\mathrm{C}_{9}\right), 136.55\left(\mathrm{C}_{10}\right), 136.33\left(\mathrm{C}_{11}\right), 135.77\left(\mathrm{C}_{12}\right)$, $129.88\left(C_{13}\right), 128.84\left(C_{14}\right), 122.94\left(C_{15}\right), 122.33\left(C_{16}\right), 121.19\left(C_{17}\right), 118.84\left(C_{18}\right)$, $108.20\left(\mathrm{C}_{19}\right), 104.05\left(\mathrm{C}_{20}\right), 53.96\left(\mathrm{C}_{21}\right), 26.36\left(\mathrm{C}_{22}\right)$, Overlapping with THF signal at $25.14\left(\mathrm{C}_{23} / \mathrm{C}_{24}\right), 21.17\left(\mathrm{C}_{25}\right)$.

${ }^{15} \mathrm{~N}$ NMR $\left({ }^{15} \mathrm{~N}\right.$ enriched sample, $\left.61 \mathrm{MHz}, 0-\mathrm{C}_{6} \mathrm{Cl}_{2} \mathrm{D}_{4}\right) \delta 291.80$ (s).

FT-IR vSc-N: $920 \mathrm{~cm}^{-1}$

\section{3-N:}

A $50 \mathrm{ml}$ thick-walled glass vessel equipped with a Kontes PTFE tap and stirbar was charged with 2-N (48mg, $0.07 \mathrm{mmol})$ and THF $(5 \mathrm{ml})$. After three freeze-pump-thaw cycles, the flask was placed under $1 \mathrm{~atm}$ of $\mathrm{CO}_{2}$, immediately turning the deep purple solution very faint yellow/colorless. After stirring 30 minutes, a white precipitate formed, which was collected on a fine porosity frit and washed with THF $(5 \mathrm{ml})$, pentane $(3 \times 5 \mathrm{ml})$, and dried in vacuo. (29mg, 54\%).

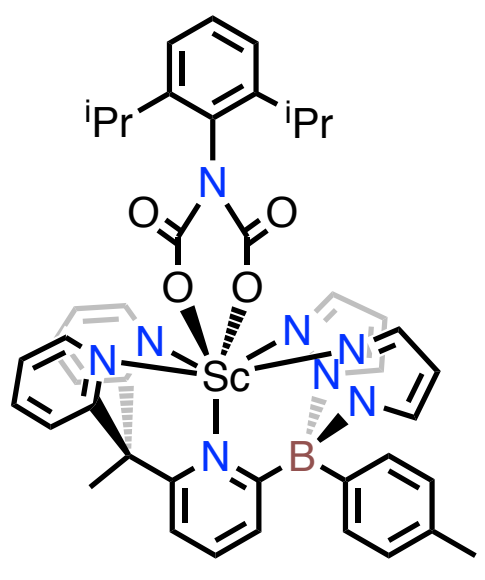


Similarly, the ${ }^{13} \mathrm{C} /{ }^{15} \mathrm{~N}$ labelled materials were synthesized using ${ }^{13} \mathrm{CO}_{2}$ and/or 2${ }^{15} \mathrm{~N}$. Elemental Analysis: Calcd. (\%) for $\left(\mathrm{C}_{44} \mathrm{H}_{44} \mathrm{BN}_{8} \mathrm{O}_{4} \mathrm{Sc}\right)$ : C, 65.68; $\mathrm{H}, 5.51 ; \mathrm{N}$, 13.93. Found: $\mathrm{C}, 62.33 ; \mathrm{H}, 5.40 ; \mathrm{N}, 12.96$. Results for $\mathrm{EA}$ are consistently low in carbon and nitrogen, presumably due to incomplete combustion. 


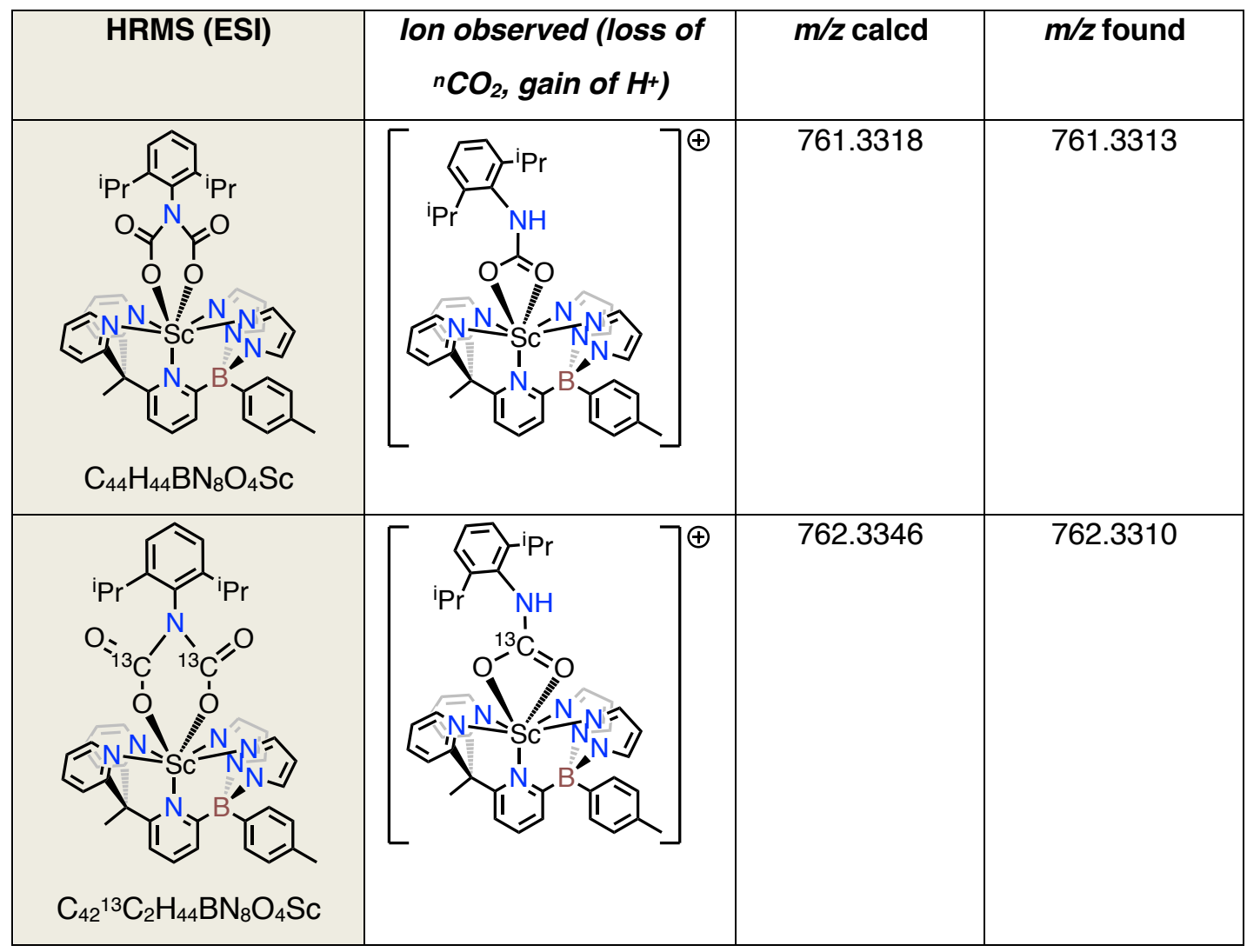

${ }^{1} \mathrm{H}$ NMR $\left(500 \mathrm{MHz}, o-\mathrm{Cl}_{2} \mathrm{C}_{6} \mathrm{D}_{4}\right) \delta 10.34\left(\mathrm{~d}, J=5.5 \mathrm{~Hz}, 2 \mathrm{H}, \mathrm{H}_{\mathrm{a}}\right), 8.92\left(\mathrm{~d}, 2 \mathrm{H}, \mathrm{H}_{\mathrm{b}}\right)$, $7.60\left(\mathrm{~d}, J=7.0 \mathrm{~Hz}, 1 \mathrm{H}, \mathrm{H}_{\mathrm{c}}\right), 7.46\left(\mathrm{~d}, J=7.0 \mathrm{~Hz}, 2 \mathrm{H}, \mathrm{H}_{\mathrm{d}}\right), 7.30$ (d, $J=8.3 \mathrm{~Hz}, 2 \mathrm{H}$, $\left.\mathrm{H}_{\mathrm{e}}\right), 7.22\left(\mathrm{t}, J=7.8 \mathrm{~Hz}, 2 \mathrm{H}, \mathrm{H}_{\mathrm{f}}\right), 7.17\left(\mathrm{~d}, 2 \mathrm{H}, \mathrm{H}_{\mathrm{g}}\right), 7.13-7.10\left(\mathrm{~m}, 3 \mathrm{H}, \mathrm{H}_{\mathrm{h}}\right), 7.07-$ $7.03\left(\mathrm{~m}, 3 \mathrm{H}, \mathrm{H}_{\mathrm{i}}\right), 6.93\left(\mathrm{~d}, J=7.7 \mathrm{~Hz}, 1 \mathrm{H}, \mathrm{H}_{\mathrm{j}}\right), 6.76\left(\mathrm{t}, J=6.0 \mathrm{~Hz}, 2 \mathrm{H}, \mathrm{H}_{\mathrm{k}}\right), 5.88(\mathrm{~d}$, $\left.J=2.4 \mathrm{~Hz}, 2 \mathrm{H}, \mathrm{H}_{\mathrm{I}}\right), 3.13$ (hept, $J=6.3 \mathrm{~Hz}, 2 \mathrm{H}, \mathrm{H}_{\mathrm{m}}$ ), $2.27\left(\mathrm{~s}, 3 \mathrm{H}, \mathrm{H}_{\mathrm{n}}\right), 2.23(\mathrm{~s}, 3 \mathrm{H}$, $\left.\mathrm{H}_{\mathrm{o}}\right), 1.04\left(\mathrm{~d}, J=6.2 \mathrm{~Hz}, 12 \mathrm{H}, \mathrm{H}_{\mathrm{p}}\right)$.

${ }^{11} \mathrm{~B}\left\{{ }^{1} \mathrm{H}\right\}$ NMR $\left(161 \mathrm{MHz}, o-\mathrm{Cl}_{2} \mathrm{C}_{6} \mathrm{D}_{4}\right)$ : peak too broad to observe.

${ }^{13} \mathrm{C}\left\{{ }^{1} \mathrm{H}\right\}$ NMR (126 MHz, o- $\left.\mathrm{Cl}_{2} \mathrm{C}_{6} \mathrm{D}_{4}\right) \delta 174.66$ (observed in $\mathrm{HMBC}$ but not in $\left.{ }^{13} \mathrm{C}\left\{{ }^{1} \mathrm{H}\right\}, \mathrm{C}_{27}\right), 157.19\left(\mathrm{C}_{1}\right), 156.59\left(\mathrm{C}_{2}\right), 153.99\left(\mathrm{C}_{3}\right), 151.45\left(\mathrm{C}_{4}\right), 144.42\left(\mathrm{C}_{5}\right)$, $142.81\left(\mathrm{C}_{6}\right), 138.37\left(\mathrm{C}_{7}\right), 137.51\left(\mathrm{C}_{8}\right), 136.02\left(\mathrm{C}_{9}\right), 134.72\left(\mathrm{C}_{10}\right), 134.53\left(\mathrm{C}_{11}\right)$, $134.32\left(\mathrm{C}_{12}\right), 133.62\left(\mathrm{C}_{13}\right), 127.27\left(\mathrm{C}_{14}\right), 126.87\left(\mathrm{C}_{15}\right), 126.77\left(\mathrm{C}_{16}\right), 121.13\left(\mathrm{C}_{17}\right)$, $120.88\left(\mathrm{C}_{18}\right), 119.12\left(\mathrm{C}_{19}\right), 116.68\left(\mathrm{C}_{20}\right), 102.14\left(\mathrm{C}_{21}\right), 50.77\left(\mathrm{C}_{22}\right), 26.84\left(\mathrm{C}_{23}\right)$, $22.07\left(\mathrm{C}_{24}\right), 19.37\left(\mathrm{C}_{25 / 26}\right)$. 
${ }^{13} \mathrm{C}\left\{{ }^{1} \mathrm{H}\right\}$ NMR $\left({ }^{13} \mathrm{C} /{ }^{15} \mathrm{~N}\right.$ enriched sample, $\left.151 \mathrm{MHz}, o-\mathrm{C}_{6} \mathrm{Cl}_{2} \mathrm{D}_{4}\right) \delta 153.98\left(\mathrm{~d},{ }^{1} \mathrm{~J}_{C N}=\right.$ $17.4 \mathrm{~Hz})$.

${ }^{15} \mathrm{~N}$ NMR $\left({ }^{13} \mathrm{C} /{ }^{15} \mathrm{~N}\right.$ enriched sample, $\left.61 \mathrm{MHz}, o-\mathrm{C}_{6} \mathrm{Cl}_{2} \mathrm{D}_{4}\right) \delta 136.85\left(\mathrm{t},{ }^{1} J_{N C}=17.3\right.$ $\mathrm{Hz})$.

FT-IR: $v \mathrm{C}=0: 1632 \mathrm{~cm}^{-1}, 1685 \mathrm{~cm}^{-1}$

4:

A $50 \mathrm{ml}$ thick-walled glass vessel equipped with a Kontes PTFE tap and stirbar was charged with $\mathrm{BPz} \mathrm{z}_{2} \mathrm{Py}_{3} \mathrm{H}$ (90mg, $\quad 0.18 \mathrm{mmol}), \quad \mathrm{Sc}\left(\mathrm{CH}_{2} \mathrm{SiMe}_{2} \mathrm{Ph}\right)_{3}(\text { thf })_{2} \quad$ (115mg, $0.18 \mathrm{mmol})$, and benzene $(5 \mathrm{ml})$, and stirred until all material dissolved, turning the solution orange. A solution of 2,6diisopropylphosphine (35mg, 0.18mmol) in benzene $(5 \mathrm{ml})$ was then added, and the mixture stirred for 5 days resulting in

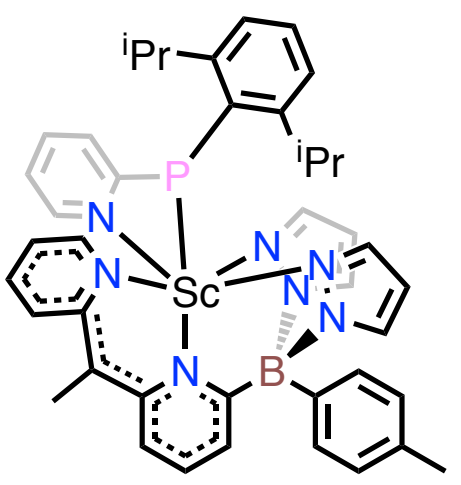
a blood-red solution. The solvent was then removed in vacuo, and the oily solid lyophilized from benzene. The dark red powder was then extracted with pentane $(4 \mathrm{ml})$, filtered through a fine porosity frit, and the solution cooled to $-50^{\circ} \mathrm{C}$ for 3 days yielding $\mathrm{x}$-ray quality red crystals that were isolated $(85 \mathrm{mg}, 58 \%)$ and stored at $50^{\circ} \mathrm{C}$. This product is thermally sensitive as a solid, and could only be isolated pure as single crystals with 1 equivalent of pentane present. Elemental Analysis: Calcd. (\%) for 4॰pentane ( $\left.\mathrm{C}_{47} \mathrm{H}_{56} \mathrm{BN} 7 \mathrm{PSC}\right)$ : C, 70.06; $\mathrm{H}, 7.01 ; \mathrm{N}, 12.17$. Found: $\mathrm{C}, 69.91$; H, 6.70; N, 12.27.

${ }^{1} \mathrm{H}$ NMR $\left(500 \mathrm{MHz}, \mathrm{C}_{6} \mathrm{D}_{6}\right) \delta 8.54\left(\mathrm{~d}, J=5.2 \mathrm{~Hz}, 1 \mathrm{H}, \mathrm{H}_{\mathrm{a}}\right), 8.04(\mathrm{~d}, J=1.8 \mathrm{~Hz}, 1 \mathrm{H}$, $\left.\mathrm{H}_{\mathrm{b}}\right), 8.01\left(\mathrm{~d}, J=7.7 \mathrm{~Hz}, 2 \mathrm{H}, \mathrm{H}_{\mathrm{c}}\right), 7.93\left(\mathrm{~d}, J=2.1 \mathrm{~Hz}, 1 \mathrm{H}, \mathrm{H}_{\mathrm{d}}\right), 7.40(\mathrm{~d}, J=5.4 \mathrm{~Hz}$, $\left.1 \mathrm{H}, \mathrm{H}_{\mathrm{e}}\right), 7.31\left(\mathrm{t}, J=7.6 \mathrm{~Hz}, 1 \mathrm{H}, \mathrm{H}_{\mathrm{f}}\right), 7.27\left(\mathrm{~d}, J=7.7 \mathrm{~Hz}, 2 \mathrm{H}, \mathrm{H}_{\mathrm{g}}\right), 7.21(\mathrm{~d}, J=7.6$ $\left.\mathrm{Hz}, 2 \mathrm{H}, \mathrm{H}_{\mathrm{h}}\right), 7.04\left(\mathrm{~d}, J=6.9 \mathrm{~Hz}, 1 \mathrm{H}, \mathrm{H}_{\mathrm{i}}\right), 6.93\left(\mathrm{dd}, J=8.7,6.9 \mathrm{~Hz}, 1 \mathrm{H}, \mathrm{H}_{\mathrm{j}}\right), 6.90-$ $6.87\left(\mathrm{~m}, 2 \mathrm{H}, \mathrm{H}_{\mathrm{k}}\right), 6.87-6.81\left(\mathrm{~m}, 2 \mathrm{H}, \mathrm{H}_{\mathrm{l}}\right), 6.62\left(\mathrm{~d}, J=8.3 \mathrm{~Hz}, 1 \mathrm{H}, \mathrm{H}_{\mathrm{m}}\right), 6.30(\mathrm{t}, J=$ $7.5 \mathrm{~Hz}, 1 \mathrm{H}, \mathrm{H}_{\mathrm{n}}$ ), $6.27\left(\mathrm{~d}, J=2.0 \mathrm{~Hz}, 1 \mathrm{H}, \mathrm{H}_{\mathrm{o}}\right), 6.23\left(\mathrm{td}, J=5.7,2.6 \mathrm{~Hz}, 1 \mathrm{H}, \mathrm{H}_{\mathrm{p}}\right.$ ), $5.75-5.69\left(\mathrm{~m}, 2 \mathrm{H}, \mathrm{H}_{\mathrm{q}}\right), 5.62\left(\mathrm{t}, \mathrm{J}=2.2 \mathrm{~Hz}, 1 \mathrm{H}, \mathrm{H}_{\mathrm{r}}\right), 4.16\left(\mathrm{~s}, 2 \mathrm{H}, \mathrm{H}_{\mathrm{s}}\right), 2.35(\mathrm{~s}, 3 \mathrm{H}$, $\left.\mathrm{H}_{\mathrm{t}}\right), 2.18\left(\mathrm{~s}, 3 \mathrm{H}, \mathrm{H}_{\mathrm{u}}\right), 1.15\left(\mathrm{~s}, 6 \mathrm{H}, \mathrm{H}_{\mathrm{v} / \mathrm{w}}\right), 0.92\left(\mathrm{~s}, 6 \mathrm{H}, \mathrm{H}_{\mathrm{v} / \mathrm{w}}\right)$. 
${ }^{11} \mathrm{~B}\left\{{ }^{1} \mathrm{H}\right\} \mathrm{NMR}\left(161 \mathrm{MHz}, \mathrm{C}_{6} \mathrm{D}_{6}\right) \delta 0.57$.

${ }^{31} \mathbf{P}$ NMR $\left(203 \mathrm{MHz}, \mathrm{C}_{6} \mathrm{D}_{6}\right) \delta-40.49$.

${ }^{13} \mathrm{C}\left\{{ }^{1} \mathrm{H}\right\}$ NMR $\left(126 \mathrm{MHz}, \mathrm{C}_{6} \mathrm{D}_{6}\right) \delta 186.20\left(\mathrm{~d}, J=36.7 \mathrm{~Hz}, \mathrm{C}_{1}\right), 168.10\left(\mathrm{br}, \mathrm{C}_{2}\right)$, 155.73 (br, C $\left.C_{3}\right), 151.70$ (d, J=3.2 Hz, C 4 ), 145.94 (d, J=6.6 Hz, C 5 ), 144.98 (d, J = $10.7 \mathrm{~Hz}, \mathrm{C}_{6}$ ), 140.50 (d, J = 5.1 Hz, C 7 ), 140.42 (br, $\left.\mathrm{C}_{34}\right) 140.02\left(\mathrm{C}_{8}\right), 137.62$ (C) $136.95\left(\mathrm{C}_{10}\right), 136.22\left(\mathrm{C}_{11}\right), 135.58\left(\mathrm{C}_{12}\right), 135.55\left(\mathrm{C}_{13}\right), 135.13\left(\mathrm{C}_{14}\right), 134.18$ (d, $\left.J=27.2 \mathrm{~Hz}, \mathrm{C}_{15}\right), 133.66\left(\mathrm{C}_{16}\right), 129.44\left(\mathrm{C}_{17}\right), 129.07\left(\mathrm{C}_{18}\right), 124.81\left(\mathrm{C}_{19}\right), 123.71$ (d, J=3.1 Hz, $\mathrm{C}_{20}$ ), $119.69\left(\mathrm{C}_{21}\right), 117.27\left(\mathrm{C}_{22}\right), 116.93\left(\mathrm{C}_{23}\right), 111.93$ (d, J = 5.4 Hz, $\mathrm{C}_{24}$ ), 109.02 ( $\left.\mathrm{C}_{25}\right), 104.22\left(\mathrm{C}_{26}\right), 104.08\left(\mathrm{C}_{27}\right), 79.99\left(\mathrm{C}_{28}\right), 34.19$ (d, J = 11.7 Hz, $\left.\mathrm{C}_{29}\right), 25.33\left(\mathrm{C}_{30}\right), 24.52\left(\mathrm{C}_{31}\right), 21.46\left(\mathrm{C}_{32}\right), 18.06\left(\mathrm{C}_{33}\right)$.

5:

Method A: A 25ml thick-walled glass vessel equipped with a Kontes PTFE tap and stirbar was charged with 4opentane $(35 \mathrm{mg}, 0.04 \mathrm{mmol})$ and benzene $(10 \mathrm{ml})$, producing a blood red solution. The vessel was then evacuated of gas via three freeze-pump-thaw cycles, then placed under $1 \mathrm{~atm}$ of $\mathrm{CO}_{2}$, immediately turning the solution a very light pink color. The solution was stirred for $15 \mathrm{~min}$,

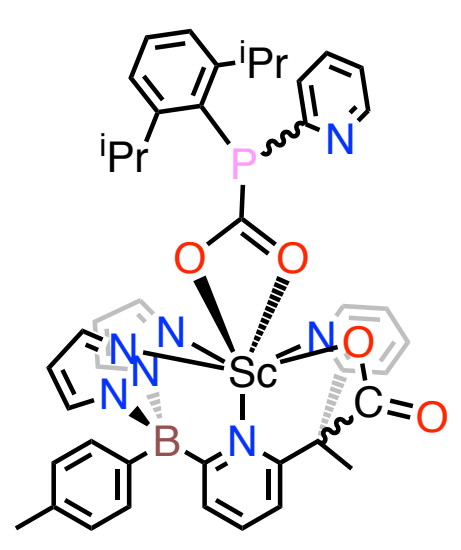
then allowed to stand at RT overnight, after which the white precipitate that was produced was collected on a fine porosity frit, washed with pentane $(3 \times 2 \mathrm{ml})$ and dried in vacuo (21mg, 59\%). Similarly, ${ }^{13} \mathrm{C}$ labelled material was generated using ${ }^{13} \mathrm{CO}_{2}$.

Method B: A 50ml thick-walled glass vessel equipped with a Kontes PTFE tap and stirbar was charged with $\mathrm{BPz}_{2} \mathrm{Py}_{3} \mathrm{H}$ (84mg, $\left.0.17 \mathrm{mmol}\right)$, $\mathrm{Sc}\left(\mathrm{CH}_{2} \mathrm{SiMe}_{2} \mathrm{Ph}\right)_{3}(\text { thf })_{2}(108 \mathrm{mg}, 0.17 \mathrm{mmol})$, and benzene $(5 \mathrm{ml})$, and stirred until all material dissolved, turning the solution orange. A solution of 2,6diisopropylphosphine (33mg, $0.17 \mathrm{mmol}$ ) in benzene $(5 \mathrm{ml})$ was then added, and the mixture stirred for 5 days resulting in a blood-red solution. The solution was 
then filtered through a fine porosity frit, and quantitatively transferred into another $50 \mathrm{ml}$ thick-walled glass vessel equipped with a Kontes PTFE tap and stirbar with additional benzene $(3 \times 2 \mathrm{ml})$. The vessel was then evacuated of gas via three freeze-pump-thaw cycles, then placed under 1 atm of $\mathrm{CO}_{2}$, gradually turning the solution an orange/pink color over $5 \mathrm{~min}$. The solution was stirred overnight, after which it was concentrated to $\sim 10 \mathrm{ml}$ in vacuo and cooled to $5^{\circ} \mathrm{C}$ for $2 \mathrm{~h}$. The white precipitate that was produced was collected on a fine porosity frit, washed with pentane $(3 \times 5 \mathrm{ml})$ and dried in vacuo $(45 \mathrm{mg}, 32 \%)$.

Elemental Analysis: Calcd. (\%) for $\left(\mathrm{C}_{44} \mathrm{H}_{44} \mathrm{BN}_{7} \mathrm{O}_{4} \mathrm{PSC}\right)$ : C, 64.32; $\mathrm{H}, 5.40$; N, 11.93. Found: C, $64.41 ; \mathrm{H}, 5.64 ; \mathrm{N}, 11.97$.

Due to the presence of both diastereomers (3:1 ratio) the $1 \mathrm{H}$ and ${ }^{13} \mathrm{C}\left\{{ }^{1} \mathrm{H}\right\}$ NMR spectra of $\mathbf{5}$ are quite complex and contain many overlapping signals, making deconvolution of peaks difficult. As a result, these could only partially be assigned:

"Major"
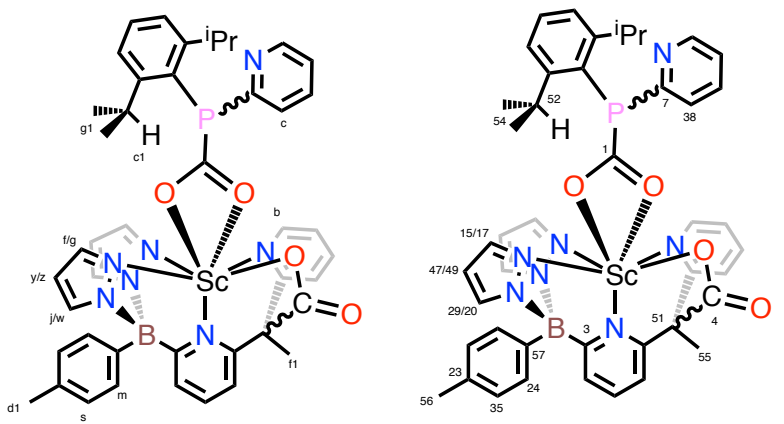

"Minor"
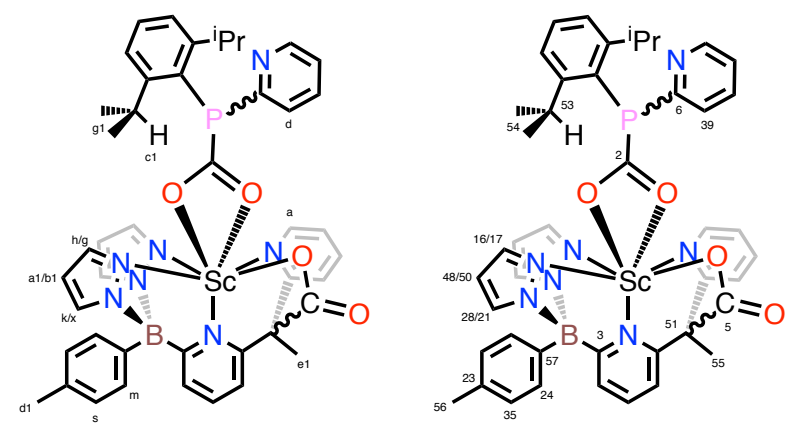

${ }^{1} \mathrm{H}$ NMR $\left(600 \mathrm{MHz}, \mathrm{THF}-\mathrm{d}_{8}\right) \delta 9.43(\mathrm{~d}, J=5.0 \mathrm{~Hz}, 1 \mathrm{H}, \mathrm{Ha}), 9.19(\mathrm{dd}, J=5.5,1.8$ $\left.\mathrm{Hz}, 3 \mathrm{H}, \mathrm{Hb}_{\mathrm{b}}\right), 8.56\left(\mathrm{~d}, J=8.5 \mathrm{~Hz}, 3 \mathrm{H}, \mathrm{H}_{\mathrm{c}}\right), 8.46\left(\mathrm{~d}, J=8.0 \mathrm{~Hz}, 1 \mathrm{H}, \mathrm{H}_{\mathrm{d}}\right), 8.38(\mathrm{~d}, J=$ 
$\left.4.3 \mathrm{~Hz}, 4 \mathrm{H}, \mathrm{H}_{\mathrm{e}}\right), 8.22\left(\mathrm{~d}, J=2.0 \mathrm{~Hz}, 3 \mathrm{H}, \mathrm{H}_{\mathrm{f}}\right), 8.21-8.18\left(\mathrm{~m}, 4 \mathrm{H}, \mathrm{H}_{\mathrm{g}}\right), 8.10(\mathrm{~d}, J=$ $\left.2.1 \mathrm{~Hz}, 1 \mathrm{H}, \mathrm{H}_{\mathrm{h}}\right), 7.96-7.92\left(\mathrm{~m}, 1 \mathrm{H}, \mathrm{H}_{\mathrm{i}}\right), 7.89\left(\mathrm{~d}, J=2.3 \mathrm{~Hz}, 3 \mathrm{H}, \mathrm{H}_{\mathrm{j}}\right), 7.87-7.83$ $\left(\mathrm{m}, 4 \mathrm{H}, \mathrm{H}_{\mathrm{k}}\right), 7.81\left(\mathrm{~d}, J=8.2 \mathrm{~Hz}, 1 \mathrm{H}, \mathrm{H}_{\mathrm{l}}\right), 7.77-7.74\left(\mathrm{~m}, 13 \mathrm{H}, \mathrm{H}_{\mathrm{m}}\right), 7.66-7.63$ $\left(\mathrm{m}, 5 \mathrm{H}, \mathrm{H}_{\mathrm{n}}\right), 7.59-7.54\left(\mathrm{~m}, 5 \mathrm{H}, \mathrm{H}_{\mathrm{o}}\right), 7.53-7.51\left(\mathrm{~m}, 4 \mathrm{H}, \mathrm{H}_{\mathrm{p}}\right), 7.48-7.44(\mathrm{~m}, 1 \mathrm{H}$, $\left.\mathrm{H}_{\mathrm{q}}\right), 7.38-7.34\left(\mathrm{~m}, 1 \mathrm{H}, \mathrm{H}_{\mathrm{r}}\right), 7.32-7.28\left(\mathrm{~m}, 14 \mathrm{H}, \mathrm{H}_{\mathrm{s}}\right), 7.15(\mathrm{dd}, J=7.9,3.0 \mathrm{~Hz}$, $\left.3 \mathrm{H}, \mathrm{H}_{\mathrm{t}}\right), 7.11-7.07\left(\mathrm{~m}, 7 \mathrm{H}, \mathrm{H}_{\mathrm{u}}\right), 7.07-7.03\left(\mathrm{~m}, 2 \mathrm{H}, \mathrm{H}_{\mathrm{v}}\right), 6.95(\mathrm{~d}, J=2.3 \mathrm{~Hz}, 3 \mathrm{H}$, $\left.\mathrm{H}_{\mathrm{w}}\right), 6.92\left(\mathrm{~d}, J=2.3 \mathrm{~Hz}, 1 \mathrm{H}, \mathrm{H}_{\mathrm{x}}\right), 6.13\left(\mathrm{t}, J=2.2 \mathrm{~Hz}, 3 \mathrm{H}, \mathrm{H}_{\mathrm{y}}\right), 6.09(\mathrm{t}, J=2.2 \mathrm{~Hz}$, $\left.3 \mathrm{H}, \mathrm{H}_{\mathrm{z}}\right), 6.07\left(\mathrm{t}, J=2.0 \mathrm{~Hz}, 1 \mathrm{H}, \mathrm{H}_{\mathrm{a} 1}\right), 6.03\left(\mathrm{t}, J=2.4 \mathrm{~Hz}, 1 \mathrm{H}, \mathrm{H}_{\mathrm{b} 1}\right), 3.79-3.59(\mathrm{~m}$, $\left.8 \mathrm{H}, \mathrm{H}_{\mathrm{c} 1}\right), 2.42\left(\mathrm{~s}, 12 \mathrm{H}, \mathrm{H}_{\mathrm{d} 1}\right), 2.20\left(\mathrm{~s}, 3 \mathrm{H}, \mathrm{H}_{\mathrm{e} 1}\right), 2.18\left(\mathrm{~s}, 9 \mathrm{H}, \mathrm{H}_{\mathrm{f} 1}\right), 0.86-0.83(\mathrm{~m}$, $\left.48 \mathrm{H}, \mathrm{H}_{\mathrm{g} 1}\right)$.

${ }^{11} \mathrm{~B}\left\{{ }^{1} \mathrm{H}\right\}$ NMR $\left(161 \mathrm{MHz}, \mathrm{THF}-\mathrm{d}_{8}\right) \delta-0.87$.

${ }^{31} \mathrm{P}\left\{{ }^{1} \mathrm{H}\right\}$ NMR $\left(203 \mathrm{MHz}, \mathrm{THF}-\mathrm{d}_{8}\right) \delta-31.59,-32.15$.

${ }^{13} \mathrm{C}\left\{{ }^{1} \mathrm{H}\right\}$ NMR $\left(151 \mathrm{MHz}\right.$, THF- $\left.d_{8}\right) \delta 198.57\left(\mathrm{~d},{ }^{1} J_{C P}=15.0 \mathrm{~Hz}, \mathrm{C}_{1}\right), 198.05\left(\mathrm{~d},{ }^{1}{ }_{C P}\right.$ $\left.=16.1 \mathrm{~Hz}, \mathrm{C}_{2}\right), 174.30\left(\mathrm{br}, \mathrm{C}_{3}\right), 169.54\left(\mathrm{C}_{4}\right), 169.43\left(\mathrm{C}_{5}\right), 163.51$ (d, J = 9.2 Hz, $\left.C_{6}\right), 162.81$ (d, J = 9.3 Hz, $\left.C_{7}\right), 161.28\left(C_{8}\right), 160.95\left(C_{9}\right), 160.20\left(C_{10}\right), 160.11\left(C_{11}\right)$, $156.45\left(C_{12}\right), 149.95\left(C_{13}\right), 142.62\left(C_{14}\right), 142.54\left(C_{15}\right), 142.13\left(C_{16}\right), 141.85\left(C_{17}\right)$, 141.02 (br, $\left.\mathrm{C}_{57}\right), 140.66\left(\mathrm{C}_{18}\right), 140.46\left(\mathrm{C}_{19}\right), 138.31\left(\mathrm{C}_{20}\right), 138.03\left(\mathrm{C}_{21}\right), 137.65$ ( $\left.\mathrm{C}_{22}\right), 137.27$ ( $\left.\mathrm{C}_{23}\right), 136.25\left(\mathrm{C}_{24}\right), 135.96\left(\mathrm{C}_{25}\right), 135.72\left(\mathrm{~d}, J=3.1 \mathrm{~Hz}, \mathrm{C}_{26}\right), 135.56$ $\left(\mathrm{C}_{27}\right), 135.42\left(\mathrm{C}_{28}\right), 135.20\left(\mathrm{C}_{29}\right), 131.43\left(\mathrm{C}_{30}\right), 131.31\left(\mathrm{C}_{31}\right), 129.57\left(\mathrm{C}_{32}\right), 129.51$ $\left(C_{33}\right), 129.29$ ( $\left.C_{34}\right), 129.17\left(C_{35}\right), 129.05\left(C_{36}\right), 128.73\left(C_{37}\right), 127.55(d, J=20.3$ $\left.\mathrm{Hz}, \mathrm{C}_{38}\right), 127.17$ (d, J = 18.0 Hz, C 39 ), $123.92\left(\mathrm{C}_{40}\right), 123.12\left(\mathrm{C}_{41}\right), 122.35$ ( $\left.\mathrm{C}_{42}\right)$, $122.04\left(\mathrm{C}_{43}\right), 121.87\left(\mathrm{C}_{44}\right), 119.58\left(\mathrm{C}_{45}\right), 119.53\left(\mathrm{C}_{46}\right), 104.69\left(\mathrm{C}_{47}\right), 104.66\left(\mathrm{C}_{48}\right)$, $104.48\left(\mathrm{C}_{49}\right), 104.42$ ( $\left.\mathrm{C}_{50}\right), 60.21$ ( $\left.\mathrm{C}_{51}\right), 34.28$ (d, $\left.\mathrm{C}_{52}\right), 34.12$ (d, $\left.\mathrm{C}_{53}\right), 23.69\left(\mathrm{C}_{54}\right)$, $22.57\left(\mathrm{C}_{55}\right), 21.15\left(\mathrm{C}_{56}\right)$.

${ }^{31} \mathrm{P}\left\{{ }^{1} \mathrm{H}\right\}$ NMR $\left({ }^{13} \mathrm{C}\right.$ enriched sample, $\left.203 \mathrm{MHz}, \mathrm{C}_{6} \mathrm{D}_{6}\right) \delta-30.66\left(\mathrm{~d},{ }^{1} \mathrm{~J}_{P C}=15.5 \mathrm{~Hz}\right.$, major product), $-31.07\left(\mathrm{~d},{ }^{1} \mathrm{~J}_{P C}=16.9 \mathrm{~Hz}\right.$, minor product).

${ }^{13} \mathrm{C}\left\{{ }^{1} \mathrm{H}\right\}$ NMR ( ${ }^{13} \mathrm{C}$ enriched sample, $\left.126 \mathrm{MHz}, \mathrm{C}_{6} \mathrm{D}_{6}\right) \delta 199.09\left(\mathrm{~d},{ }^{1} \mathrm{~J}_{C P}=15.6 \mathrm{~Hz}\right.$, major), 198.64 (d, ${ }^{1} J_{C P}=16.8 \mathrm{~Hz}$, minor), 169.88 (s, major), 169.82 (s, minor). 


\section{Supporting Figures}

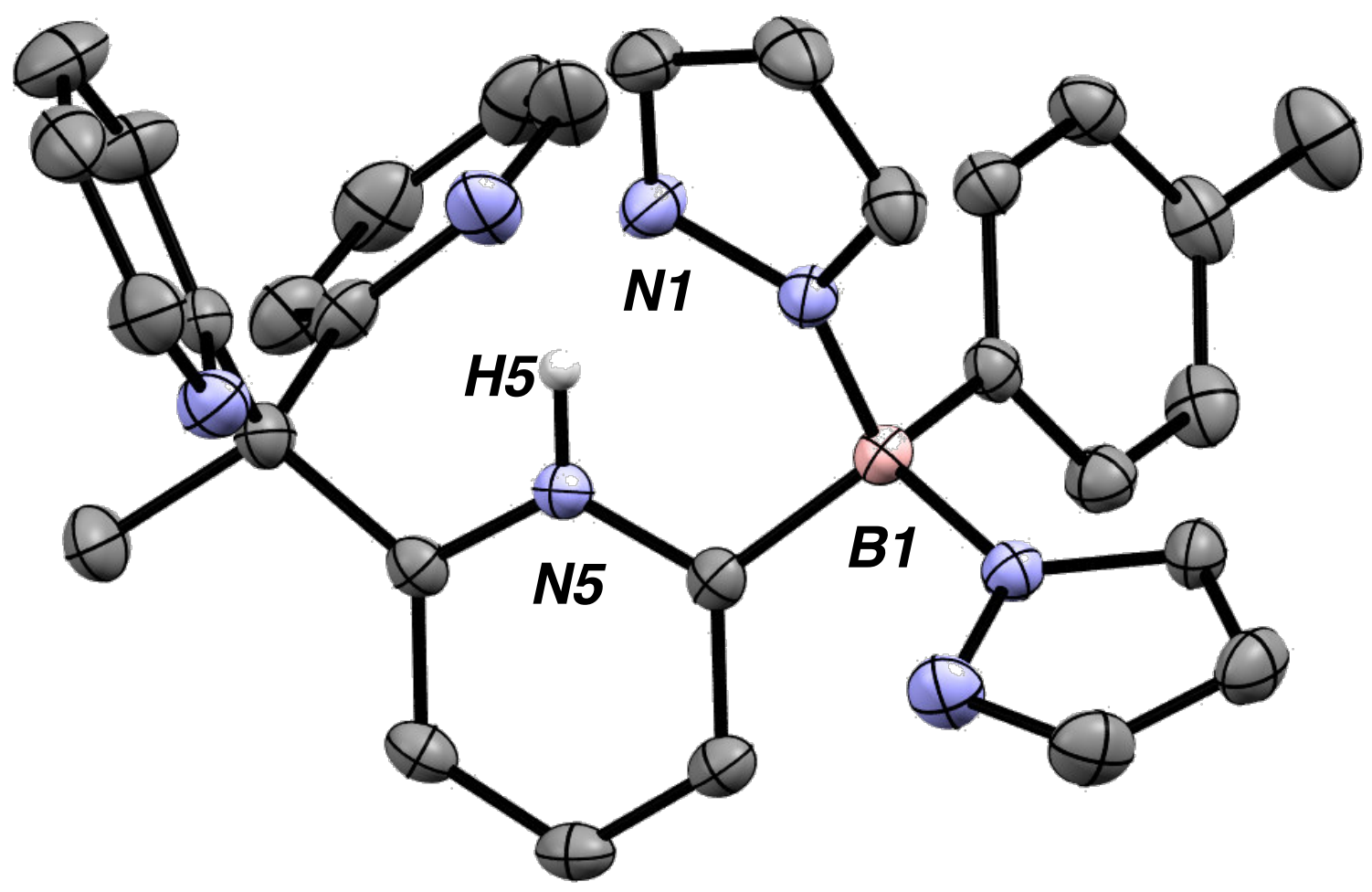

Figure S2 - Molecular structure of $\mathrm{BPz}_{2} \mathrm{Py}_{3} \mathrm{H}$. Most hydrogen atoms have been omitted for clarity. Thermal ellipsoids are shown at the $50 \%$ probability level. Selected bond length (Å) for $\mathrm{BPz}_{2} \mathrm{Py} \mathrm{y}_{3} \mathrm{H}$ : N1-H5, 1.86(2) 
0 hrs

$20 \mathrm{hrs}$

$43 \mathrm{hrs}$

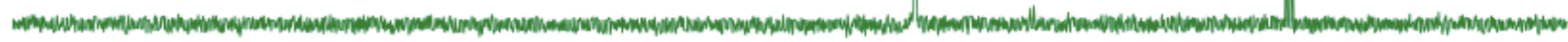

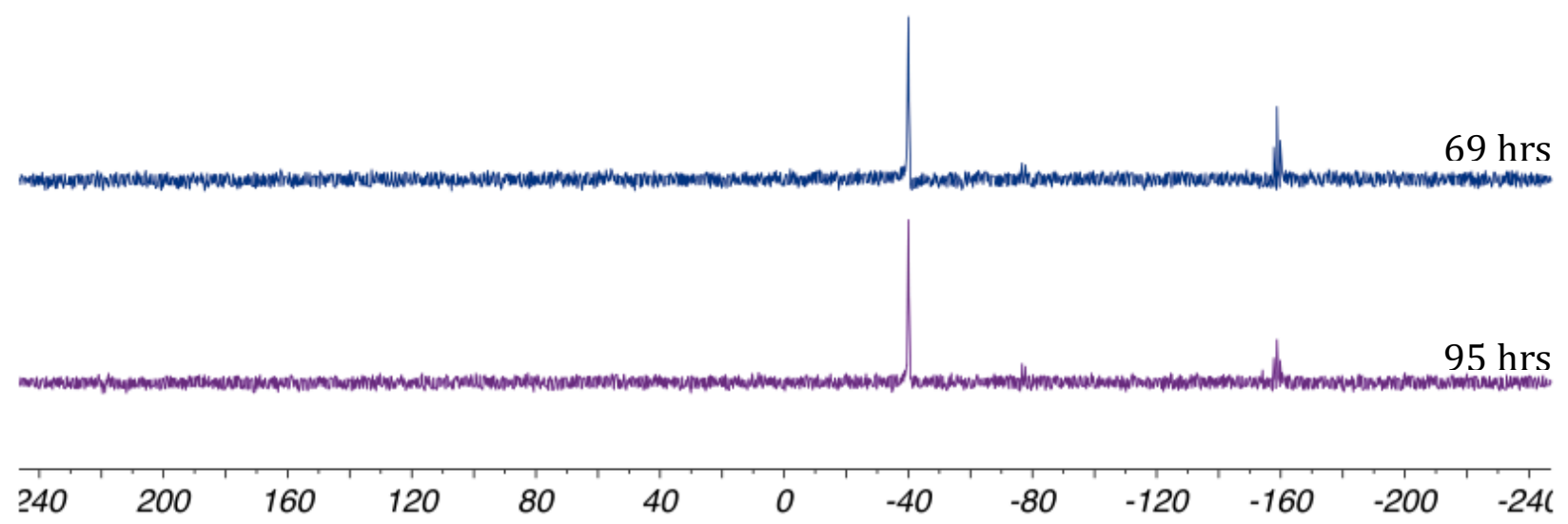

Figure S3 - Monitoring reaction progress of 1 + 2,6-diisopropylphosphine by ${ }^{31}$ P NMR in toluene-d $d_{8 .}$ Loss of 2,6-diisopropylphosphine starting material at $-158.8 \mathrm{ppm}\left(t,{ }^{1} \mathrm{~J}_{P H}=203.6 \mathrm{~Hz}\right)$ produces an intermediate doublet at $-77.2 \mathrm{ppm}\left(\mathrm{d},{ }^{1} \mathrm{JPH}=221.0 \mathrm{~Hz}\right)$, which is quickly consumed to yield the product 4 at $-40.5 \mathrm{ppm}$. 


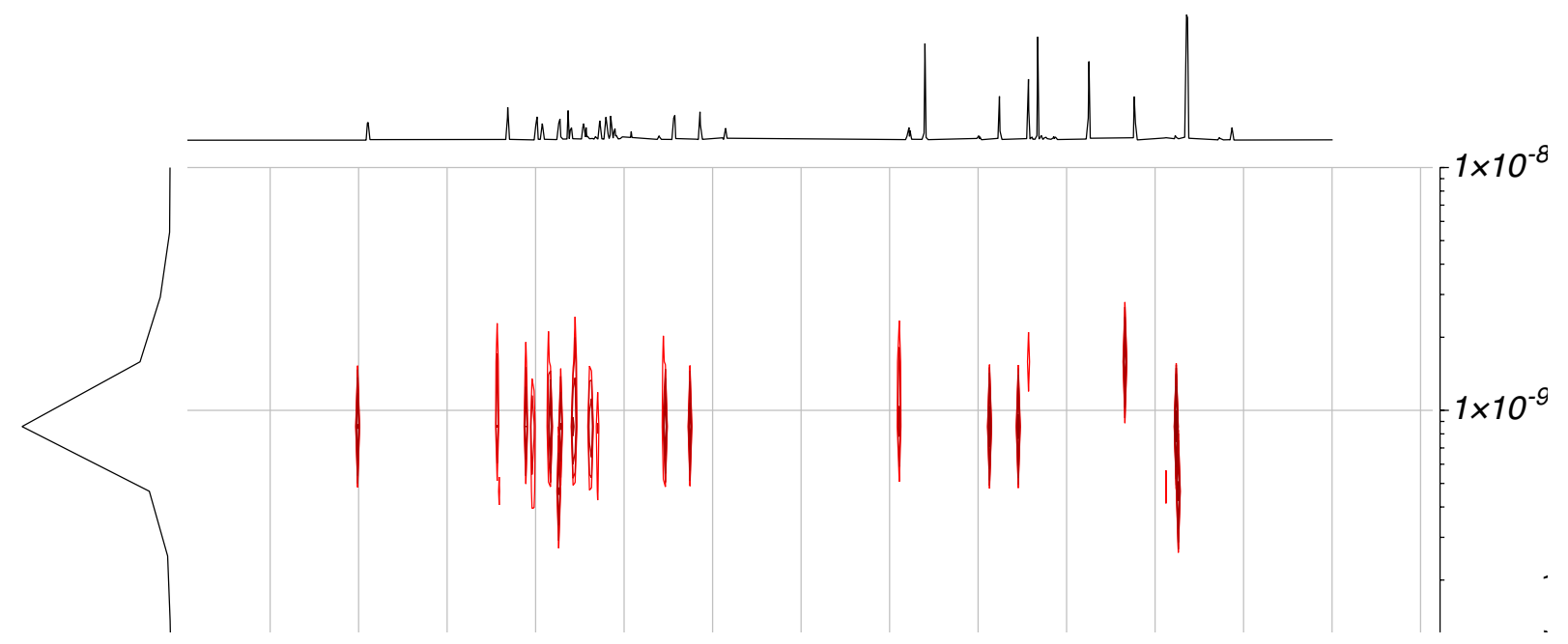

Figure S4 - 2D DOSY spectrum of 2-N at approx. 0.02M in thf-d $d_{8}$ at $298 \mathrm{~K}$. The gradient amplitude was varied from $2 \%$ to $95 \%$ with an optimized $\delta$ (gradient pulse length) of $2000 \mu$ s and a $\Delta$ (diffusion time) of $75 \mathrm{~ms}$. Diffusion coefficient was measured as $8.5 \times 10^{-10} \mathrm{~m}^{2} \mathrm{~s}^{-1}$ (units of vertical axis are $\mathrm{m}^{2} \mathrm{~s}^{-1}$ ).

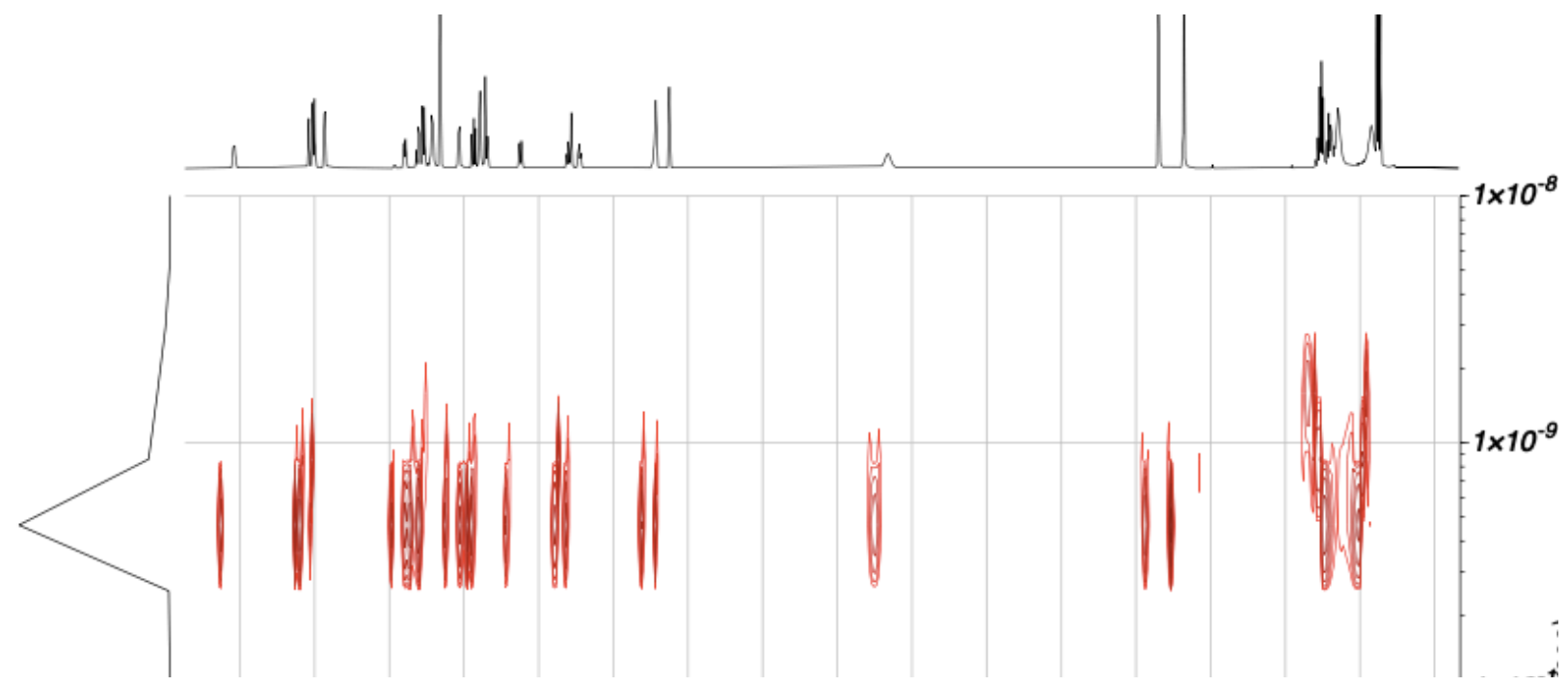

Figure S5 - 2D DOSY spectrum of 4 at approx. $0.02 \mathrm{M}$ in $C_{6} D_{6}$ at $298 \mathrm{~K}$. The gradient amplitude was varied from $2 \%$ to $95 \%$ with an optimized $\delta$ (gradient pulse length) of $2500 \mu$ s and a $\Delta$ (diffusion time) of $75 \mathrm{~ms}$. Diffusion coefficient was measured as $6.7 \times 10^{-10} \mathrm{~m}^{2} \mathrm{~s}^{-1}$ (units of vertical axis are $\mathrm{m}^{2} \mathrm{~s}^{-1}$ ). 


\begin{tabular}{c|c|c} 
Compound & $\boldsymbol{D}\left(\times \mathbf{1 0}^{-\mathbf{1 0}} \mathbf{m}^{\mathbf{2}} \mathbf{s}^{-\mathbf{1}}\right)$ & $\boldsymbol{r}(\AA)$ \\
\hline $\mathbf{2}-\mathbf{N}$ & $8.5 \pm 0.5$ & $5.3 \pm 0.7$ \\
\hline $\mathbf{4}$ & $6.7 \pm 0.4$ & $5.1 \pm 0.5$ \\
\hline $\mathbf{2} \mathbf{s c}^{-} \mathbf{H}^{*}$ & $4.8 \pm 0.5$ & $7.1 \pm 0.7$ \\
\hline $\mathbf{2 s c}_{\mathbf{S c}}-\mathbf{M e}^{*}$ & $6.9 \pm 0.5$ & $5.0 \pm 0.7$ \\
\hline 3sc $^{*}$ & $4.8 \pm 0.5$ & $7.1 \pm 0.7$ \\
\hline
\end{tabular}

Table S1 - Diffusion coefficients determined from 2D DOSY NMR, and the respective hydrodynamic radii calculated using the Stokes-Einstein equation. Estimated standard error was obtained from the width of a Gaussian lineshape in the diffusion domain. ${ }^{43}$ All samples were measured in $C_{6} D_{6}$, with the exception of 2-N which was measured in thf- $d_{8}$. Viscosity of $C_{6} D_{6}\left(0.6392 \mathrm{mPa} \cdot{ }^{4}\right)^{44}$ and THF (0.4766 mPaes) ${ }^{45}$ solvent at $298 \mathrm{~K}$ were used. Values for other dimeric $\left(2 s_{c}-\mathrm{H}, 3 \mathrm{sc}\right)$ and monomeric $(2 \mathrm{sc}-\mathrm{Me}) \mathrm{Sc}$ complexes of the related $\left[\mathrm{B}_{2} P \mathrm{z}_{4} P y\right]$ ligand are included for comparison. ${ }^{17}$ 
$\boldsymbol{A}$

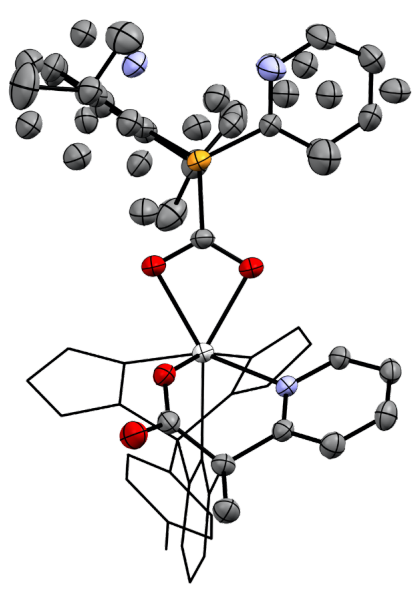

B

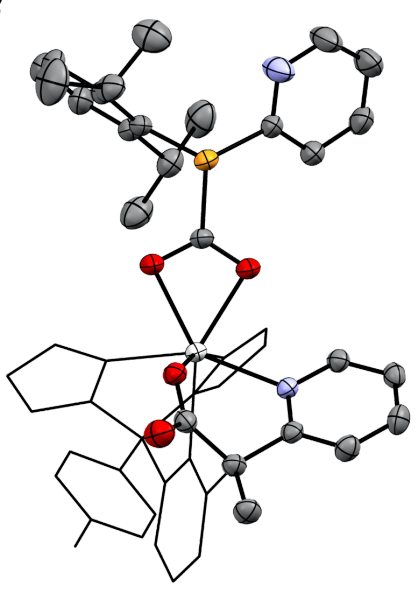

c

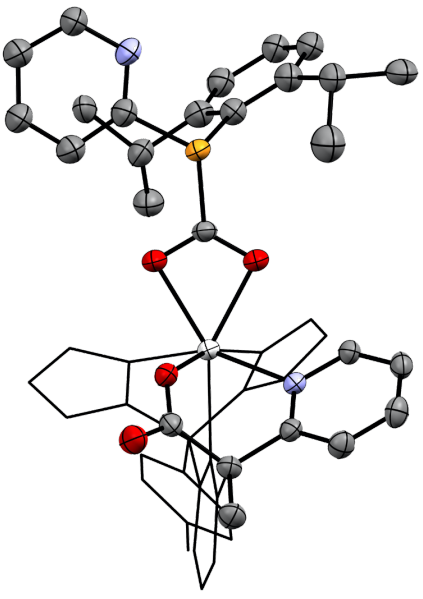

Figure S6 - Molecular structure of 5 (A, complete disordered structure) and the major (B, 92\%) and minor $(C, 8 \%)$ components that were modeled. Hydrogen atoms omitted and borate fragment of ligand depicted as wire-frame for clarity. Thermal ellipsoids are shown at the $50 \%$ probability level.
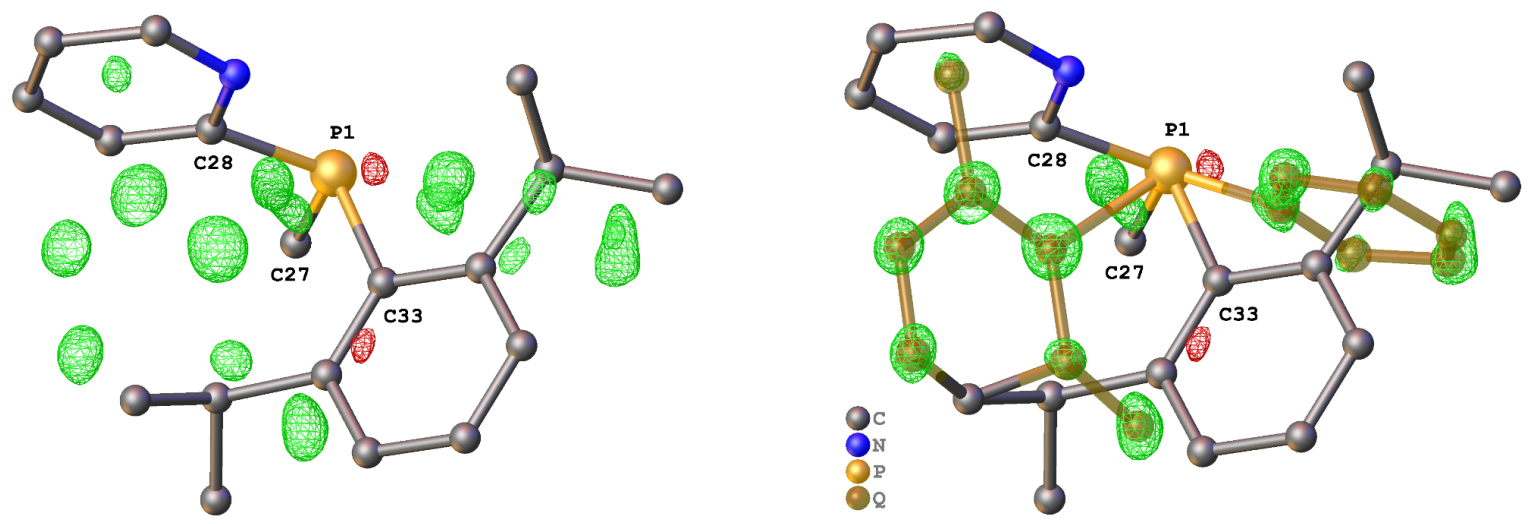

Figure S7 - Left: Residual electron density around the phosphine in 5 (prior to assignment of the disorder). Right: residual electron density around the phosphine in 5, and the corresponding Q-peaks, which highlights the presence of the opposite diastereomer. The remainder of 5 and hydrogen atoms have been omitted for clarity. 


\section{Characterization Data}

\section{NMR Data}<smiles>Cc1ccc(B(Cl)Cl)cc1</smiles>

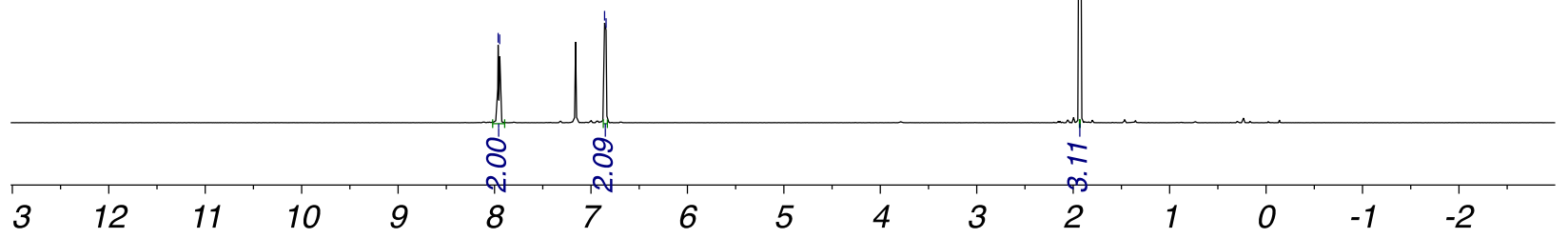

Figure $S 8{ }^{1} \mathrm{H}$ NMR of dichlorotolylborane in $C_{6} D_{6}$

搌

i

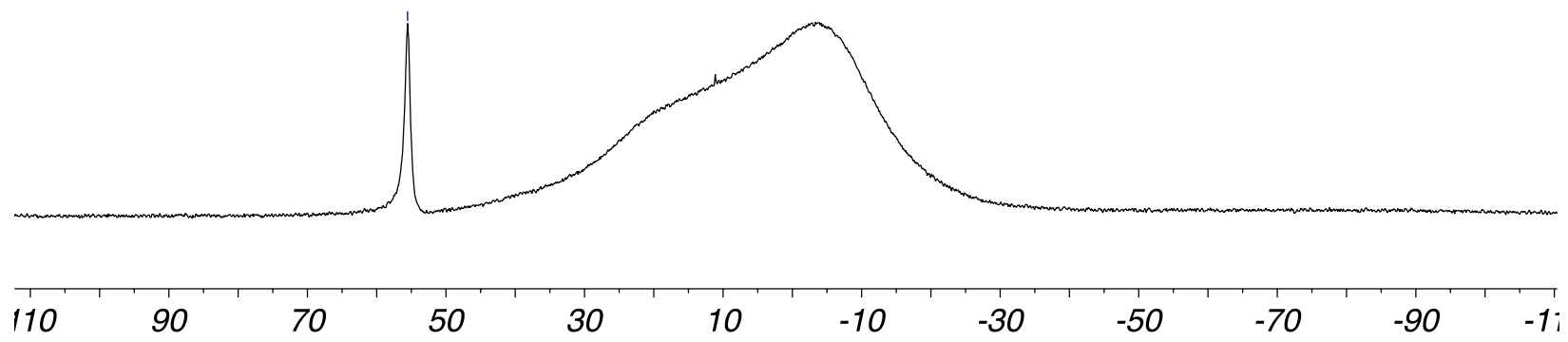

Figure S9 ${ }^{11} B$ NMR of dichlorotolylborane in $C_{6} D_{6}$. 


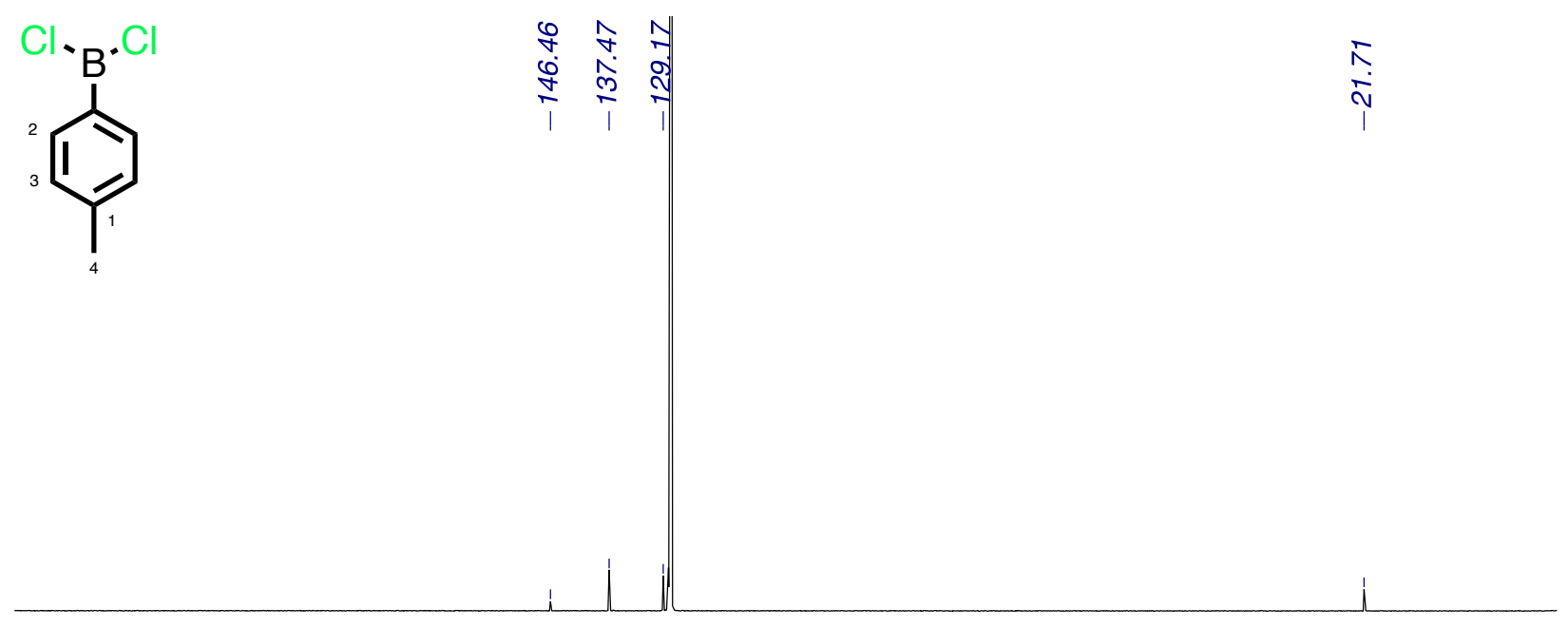

$22021020019018017016015014013012011010090 \quad 80 \quad 70 \quad 60 \quad 50 \quad 40 \quad 30 \quad 20 \quad 10 \quad 0$ Figure $S 10{ }^{13} \mathrm{C}\left\{{ }^{1} \mathrm{H}\right\}$ NMR of dichlorotolylborane in $\mathrm{C}_{6} \mathrm{D}_{6}$. 


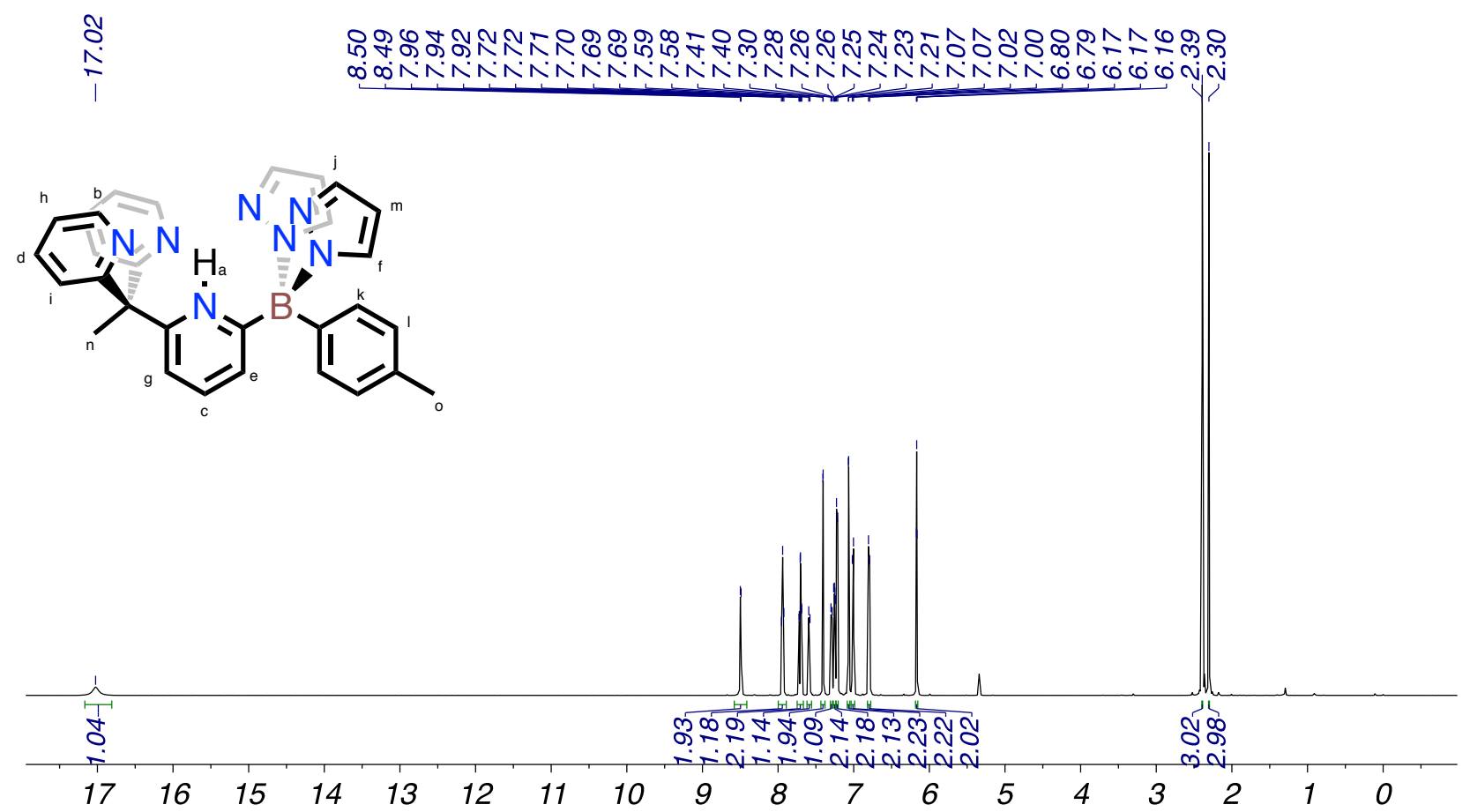

Figure $\mathrm{S} 11^{1} \mathrm{H} \mathrm{NMR}$ of $\mathrm{BPz}_{2} \mathrm{Py}_{3} \mathrm{H}$ in $\mathrm{CD}_{2} \mathrm{Cl}_{2}$
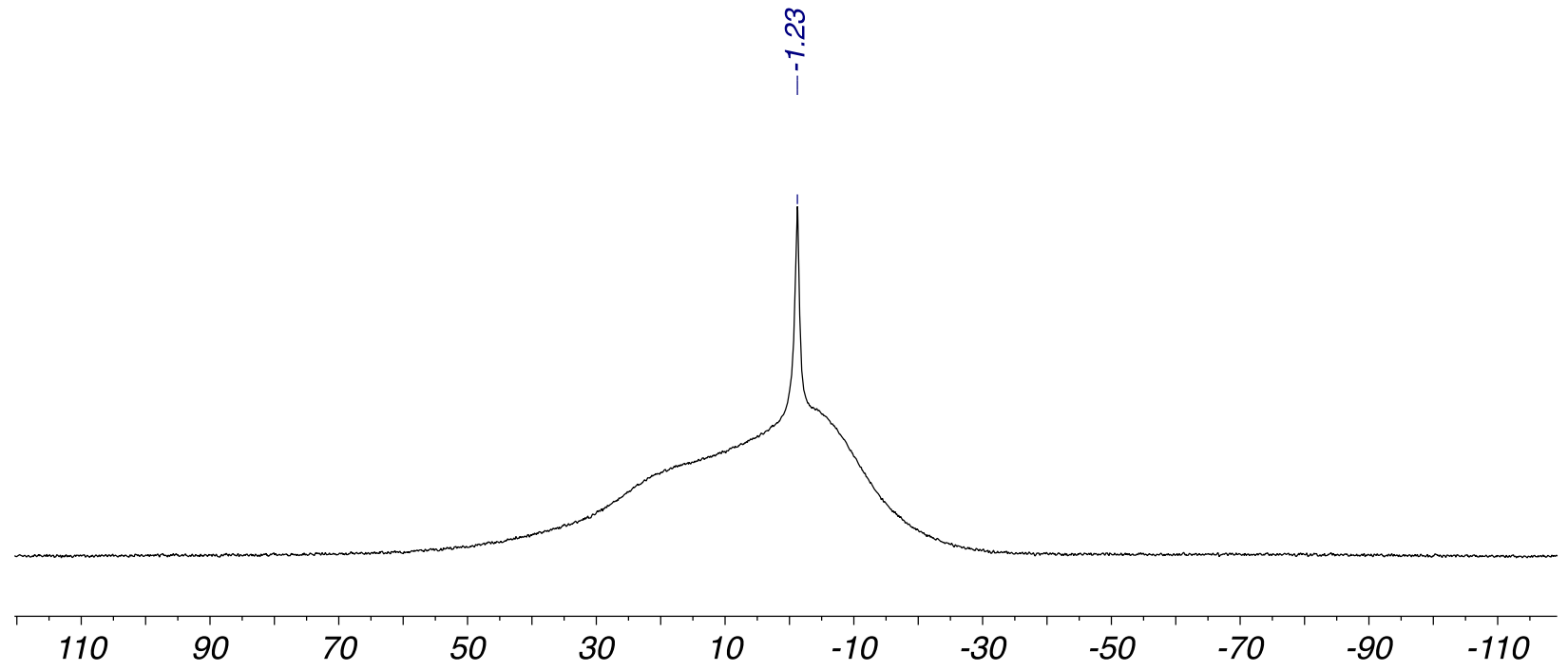

Figure $S 12{ }^{11} \mathrm{~B} N M R$ of $\mathrm{BPz}_{2} \mathrm{Py}_{3} \mathrm{H}$ in $\mathrm{CD}_{2} \mathrm{Cl}_{2}$ 


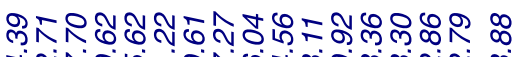

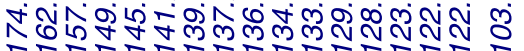
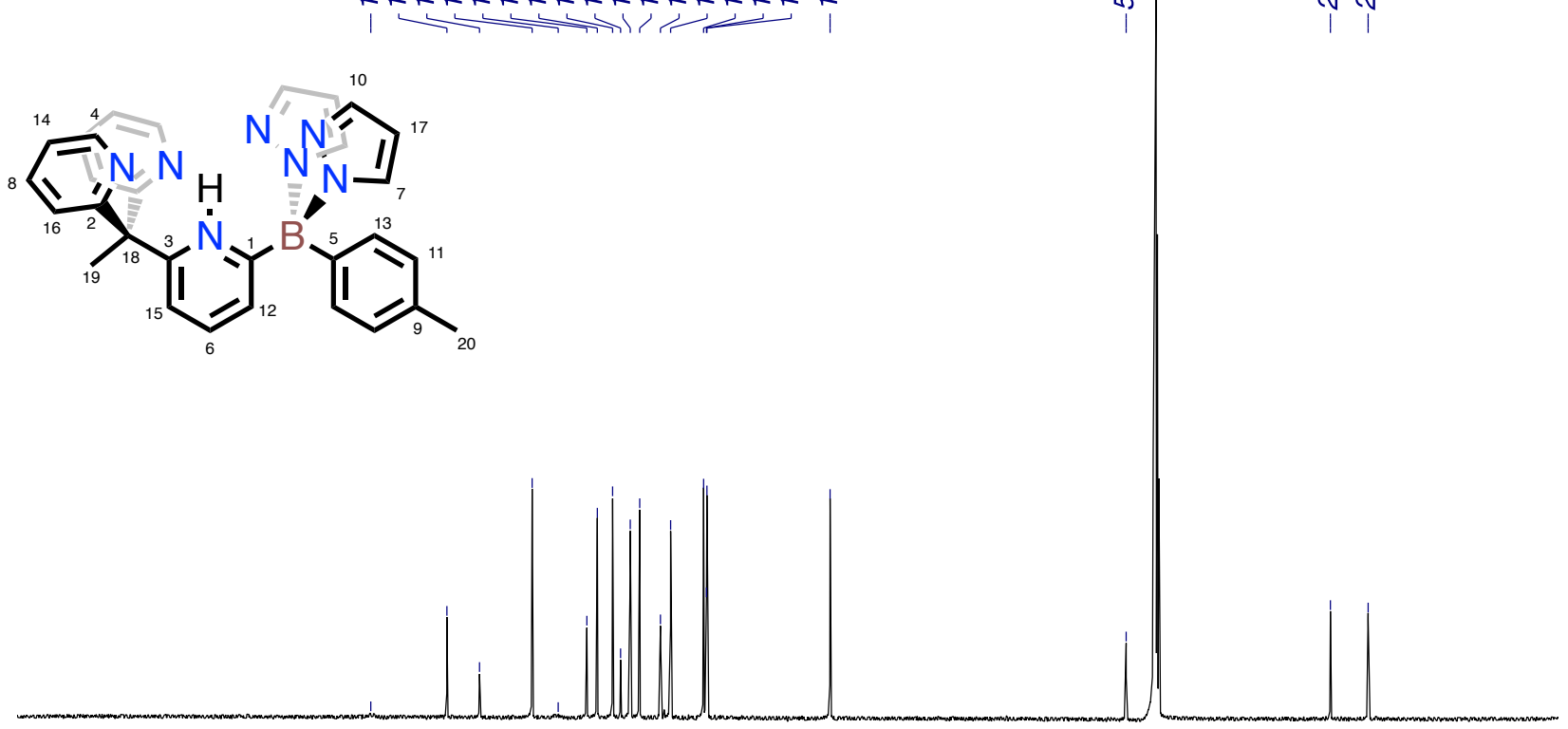

$220210200190180170160150140130120110100 \quad 90 \quad 80 \quad 70 \quad 60 \quad 50 \quad 40 \quad 30 \quad 20 \quad 10 \quad 0$

Figure $\mathrm{S}_{13}{ }^{13} \mathrm{C}_{2}{ }^{\mathrm{H}} \mathrm{H} \mathrm{NMR}$ of $\mathrm{BPz}_{2} \mathrm{Py}_{3} \mathrm{H}$ in $\mathrm{CD}_{2} \mathrm{Cl}_{2}$ 
舟

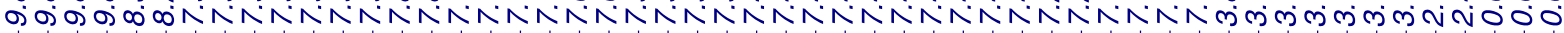
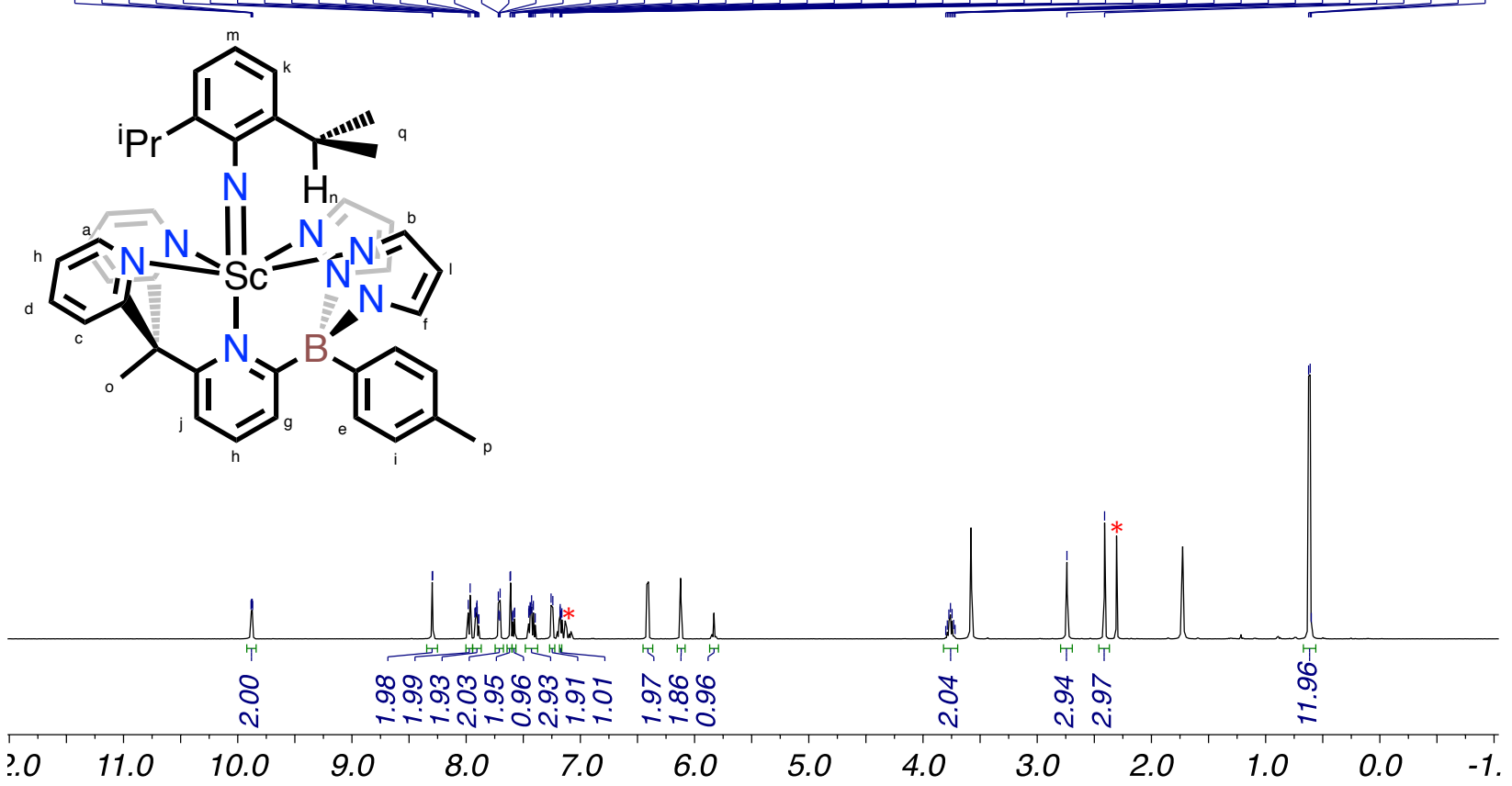

Figure S14 ${ }^{1} \mathrm{H}$ NMR of 2-N in THF-d8. Residual toluene denoted with *.

0
$\dot{7}$
$i$

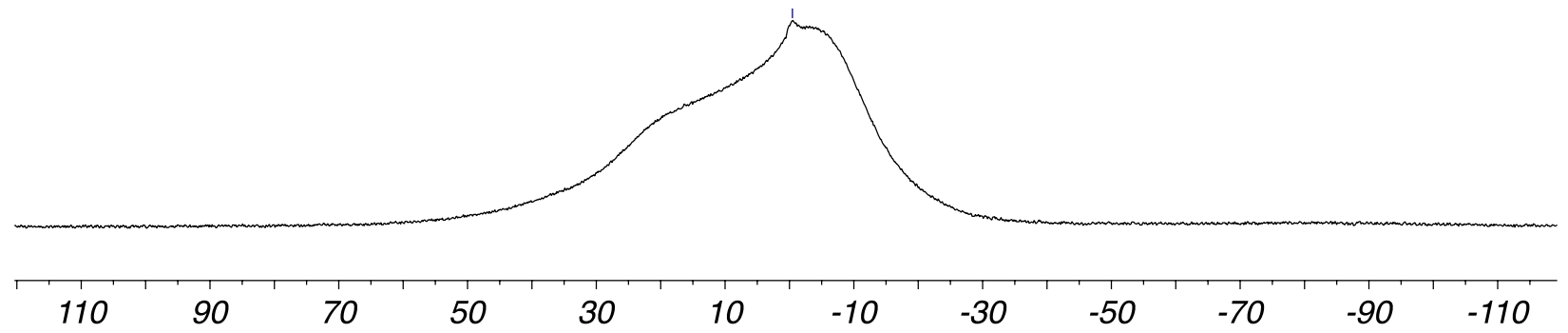

Figure S15 ${ }^{11 B}$ NMR of 2-N in THF-d 


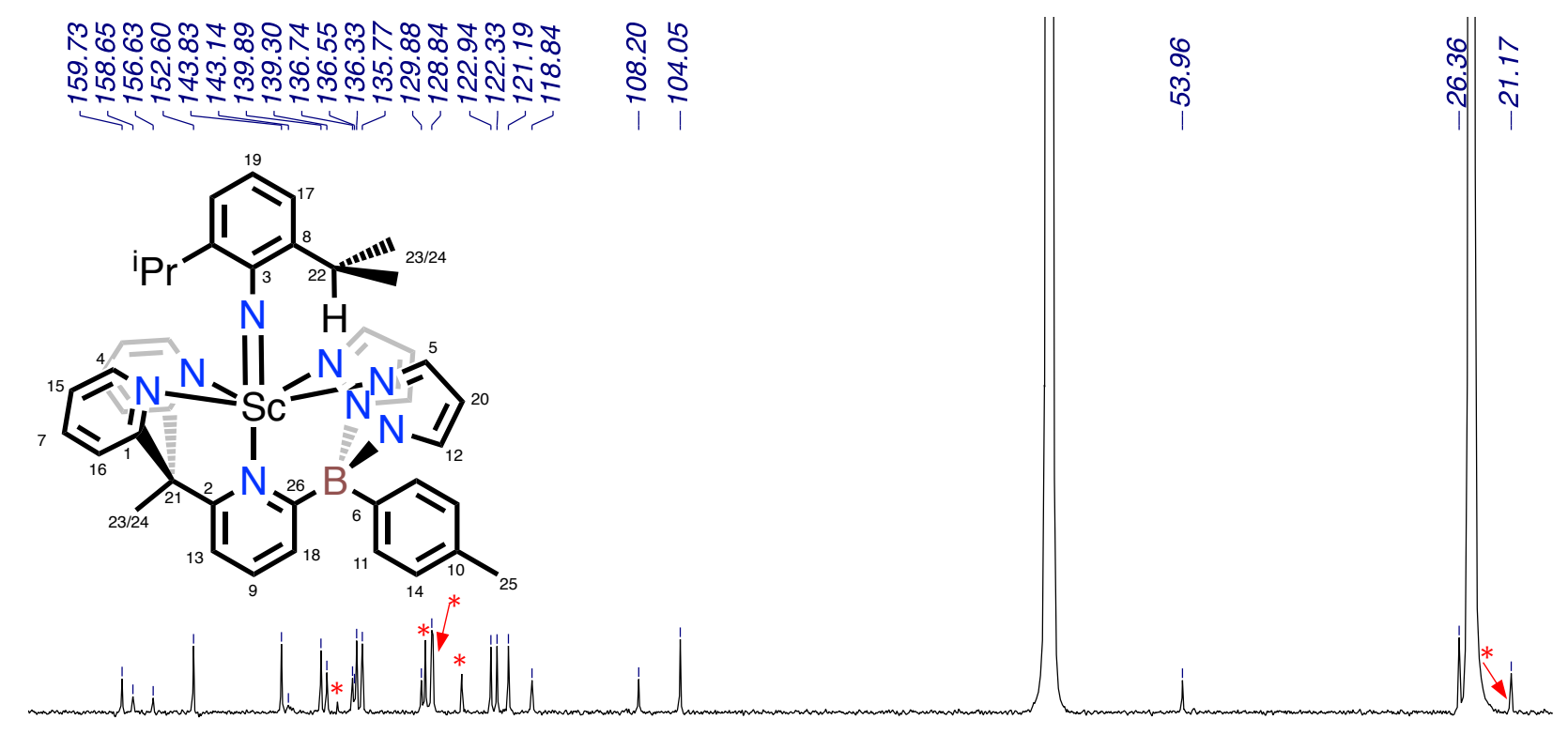

$\begin{array}{lllllllllllllll}160 & 150 & 140 & 130 & 120 & 110 & 100 & 90 & 80 & 70 & 60 & 50 & 40 & 30 & 20\end{array}$

Figure $S 16{ }^{13} \mathrm{C}\left\{{ }^{1} \mathrm{H}\right\}$ NMR of 2-N in THF-d8. Residual toluene denoted with *. 


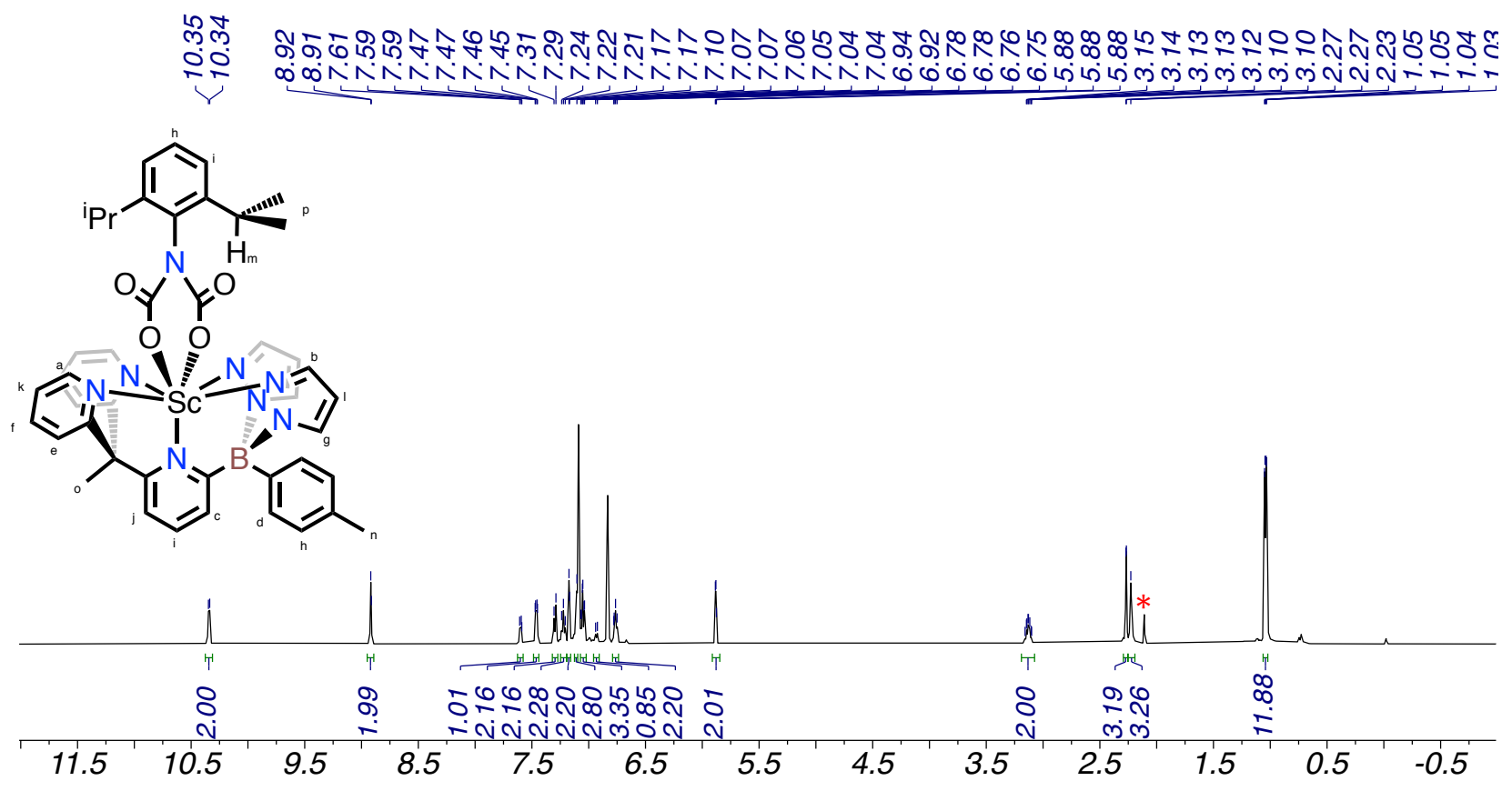

Figure $\mathrm{S}^{1}{ }^{1} \mathrm{H} \mathrm{NMR}$ of $3-\mathrm{N}$ in ${ }^{0}-\mathrm{Cl}_{2} \mathrm{C}_{6} \mathrm{D}_{4}$. Residual toluene denoted with *

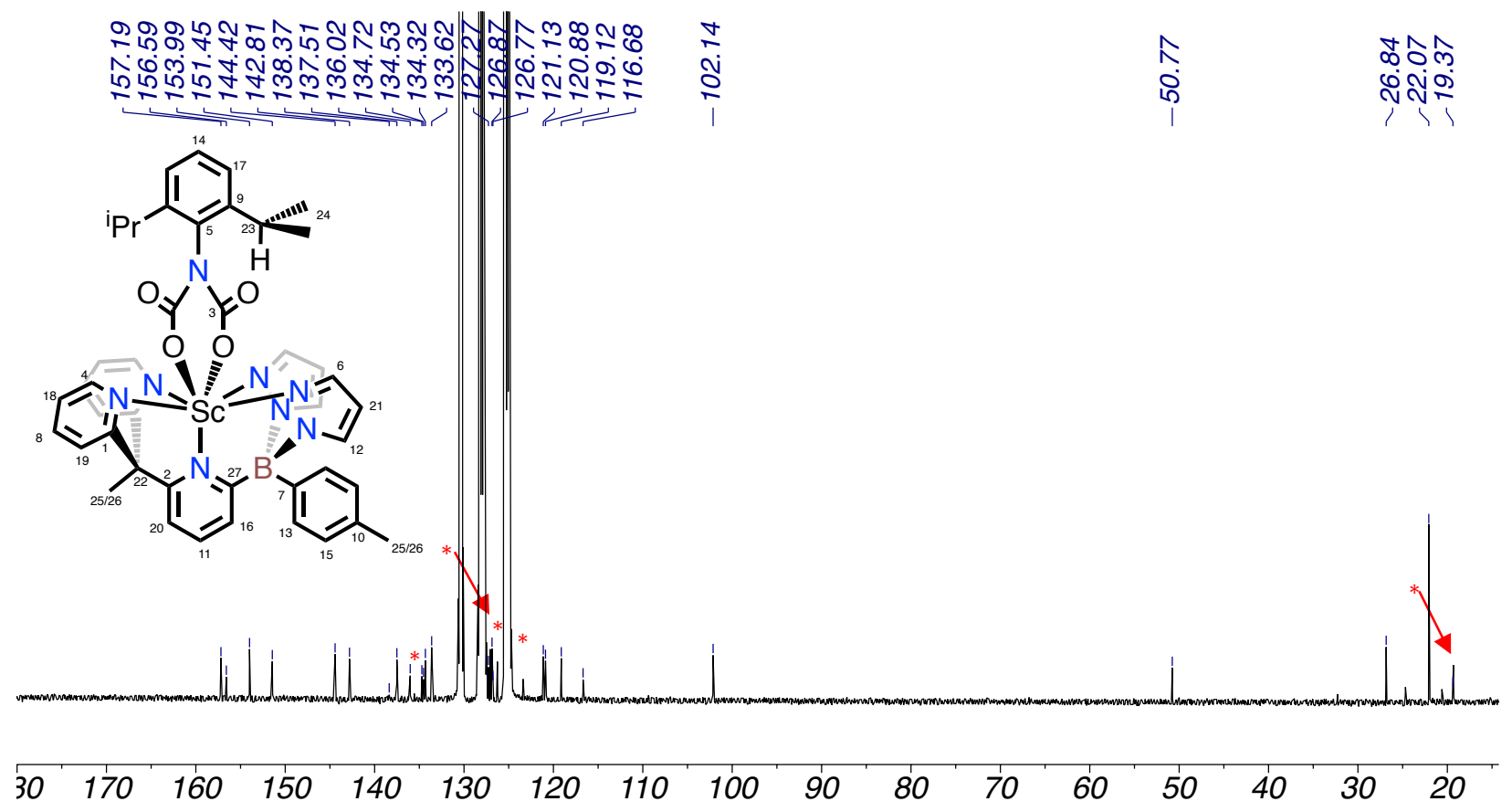

Figure $\mathrm{S}_{18}{ }^{13} \mathrm{C}\left\{{ }^{1} \mathrm{H}\right\} \mathrm{NMR}$ of 3-N in o- $\mathrm{Cl}_{2} \mathrm{C}_{6} \mathrm{D}_{4}$. Residual toluene denoted with *. 


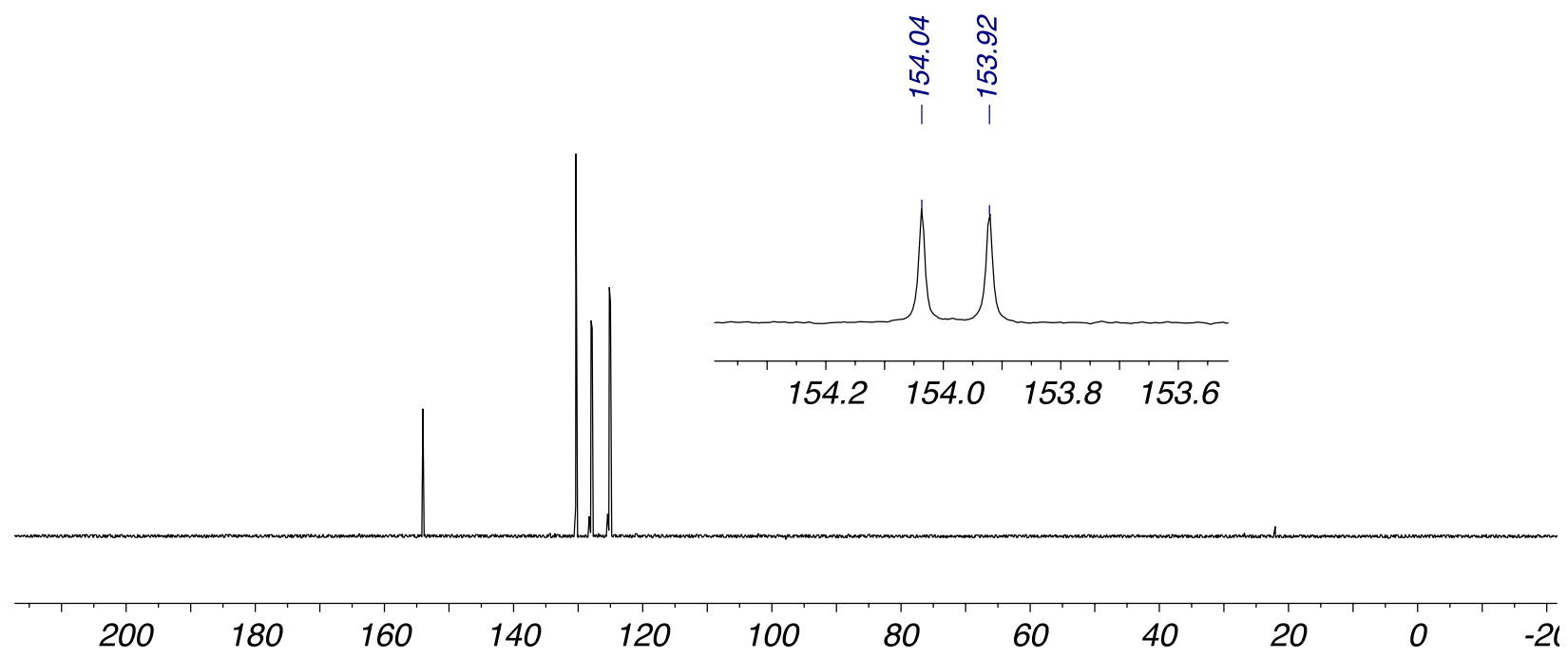

Figure $S 19{ }^{13} \mathrm{C}\left\{{ }^{1} \mathrm{H}\right\} \mathrm{NMR}$ of ${ }^{2-15} \mathrm{~N}+{ }^{13} \mathrm{CO}_{2}$ in o- $\mathrm{C}_{6} \mathrm{Cl}_{2} \mathrm{D}_{4}$.

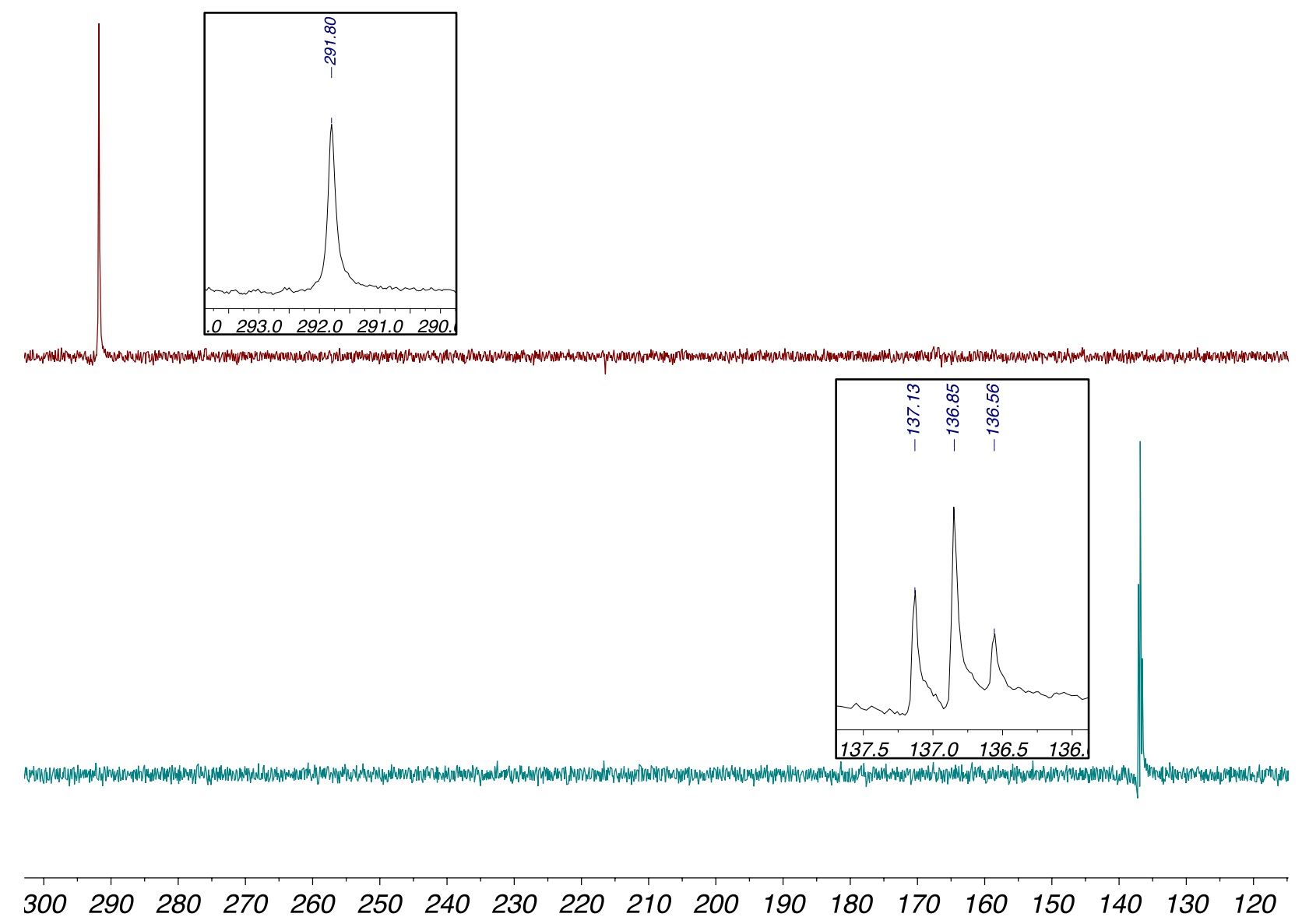

Figure S20 ${ }^{15} \mathrm{~N} \mathrm{NMR}$ of $2-{ }^{-15} \mathrm{~N}$ (top), and after addition of ${ }^{13} \mathrm{CO}_{2}$ (bottom) in o- $\mathrm{C}_{6} \mathrm{Cl}_{2} \mathrm{D}_{4}$. 


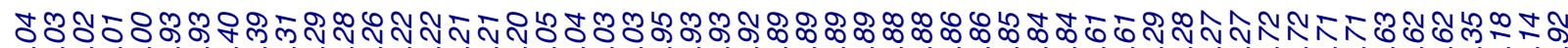

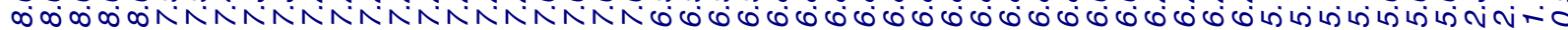

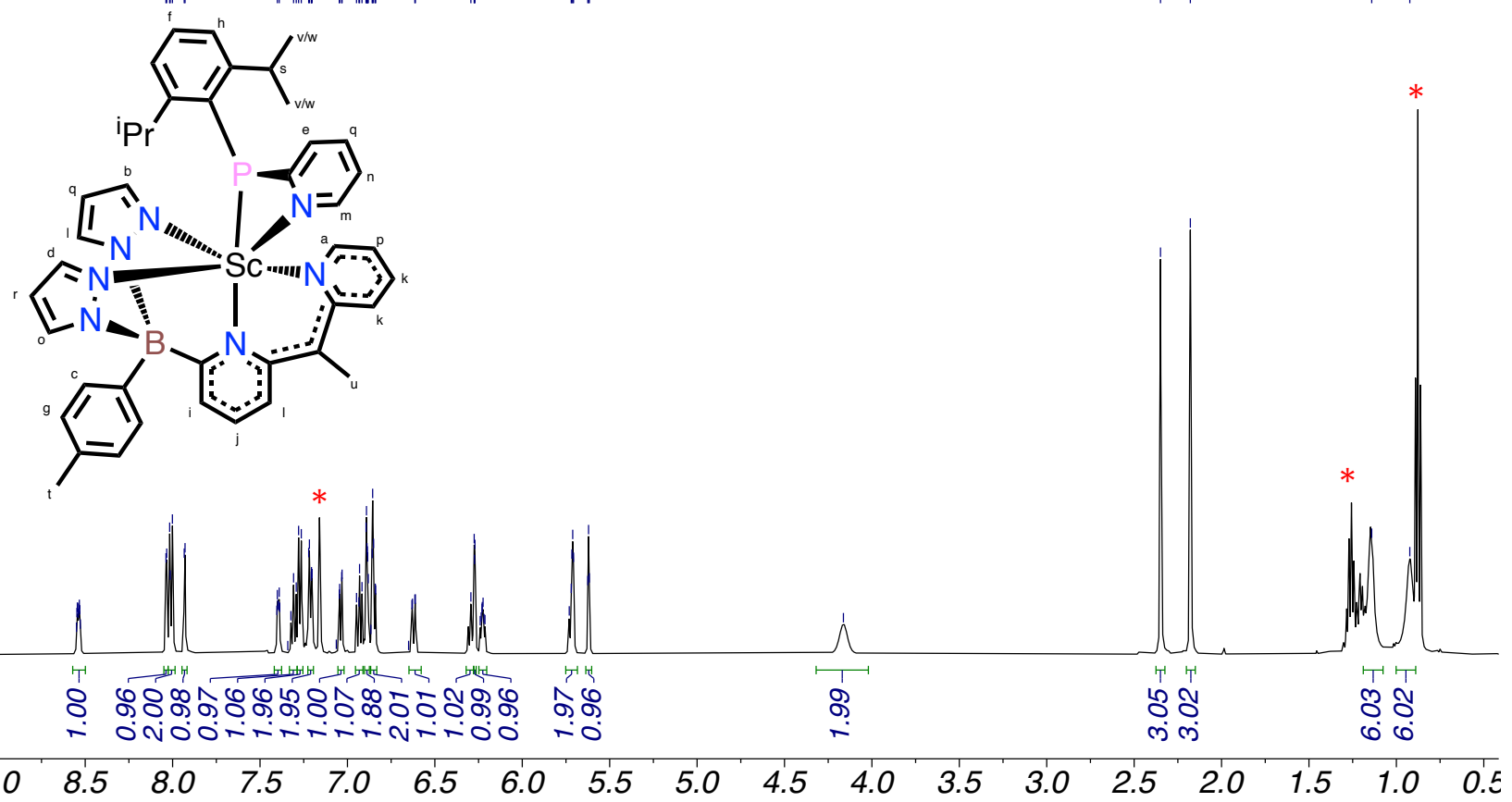

Figure S21 ${ }^{1} \mathrm{H}$ NMR of 4. Residual pentane and benzene solvent signals denoted with *.

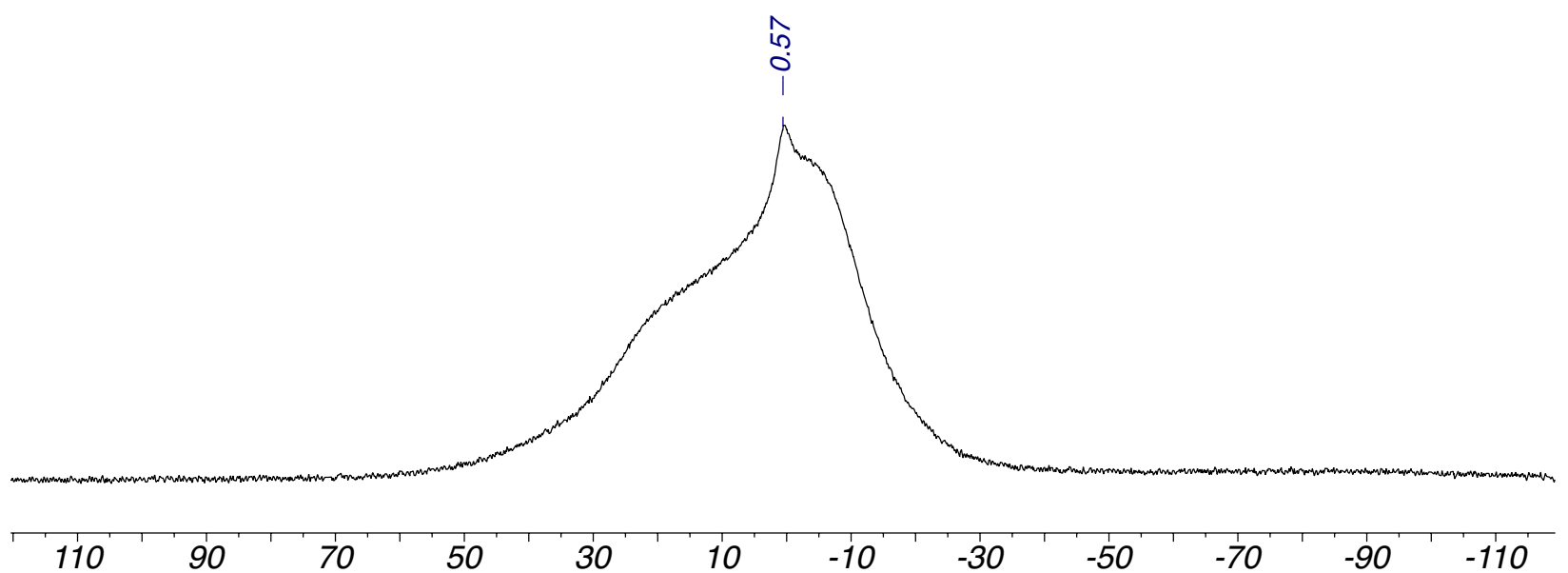

Figure $S 22{ }^{11} B\left\{{ }^{1} \mathrm{H}\right\}$ NMR of 4. 


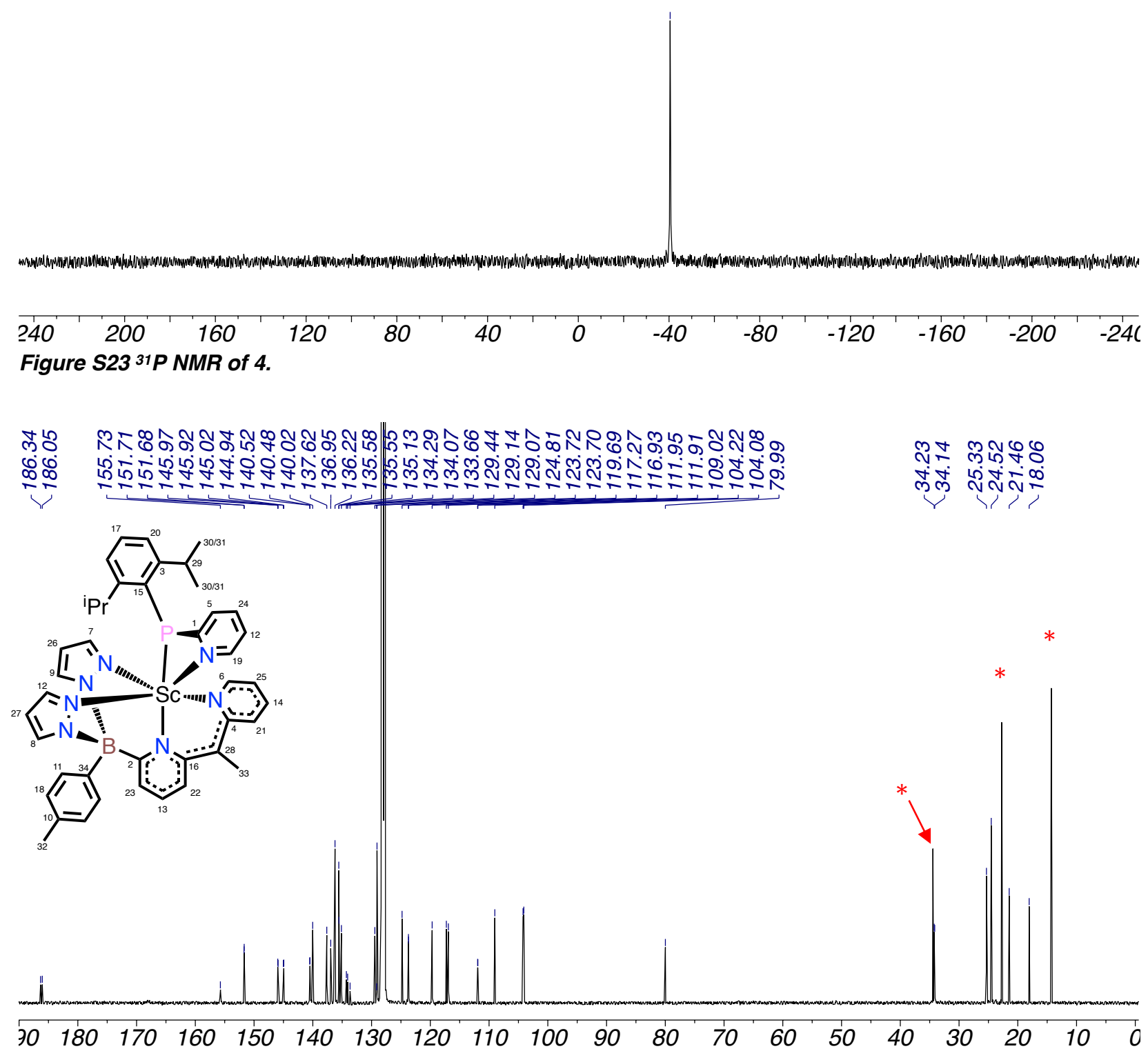
Figure $S 24{ }^{13} \mathrm{C}\left\{{ }^{1} \mathrm{H}\right\}$ NMR of 4. Residual pentane denoted with * 


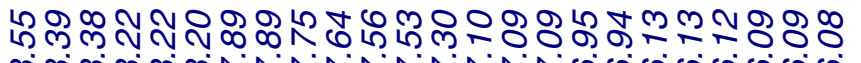
0000000

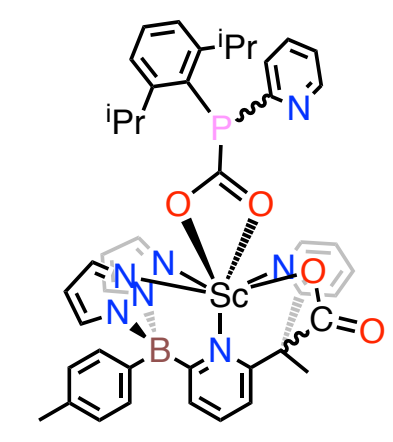

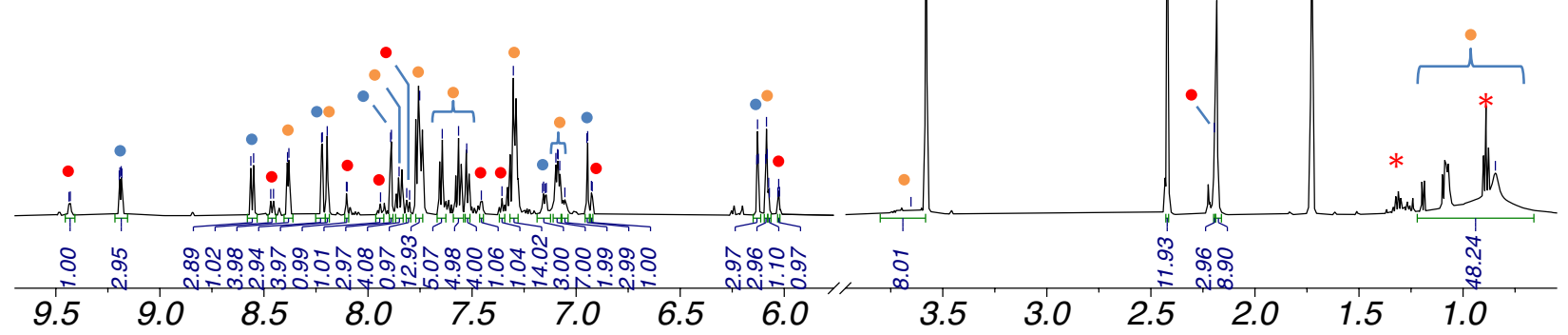

Figure S25 - ${ }^{1} \mathrm{H}$ NMR of 5 in THF-d $d_{8}$. Blue and red markers denote signal from major or minor diastereomer, respectively. Green markers denote overlapping signals. Residual pentane denoted with *.

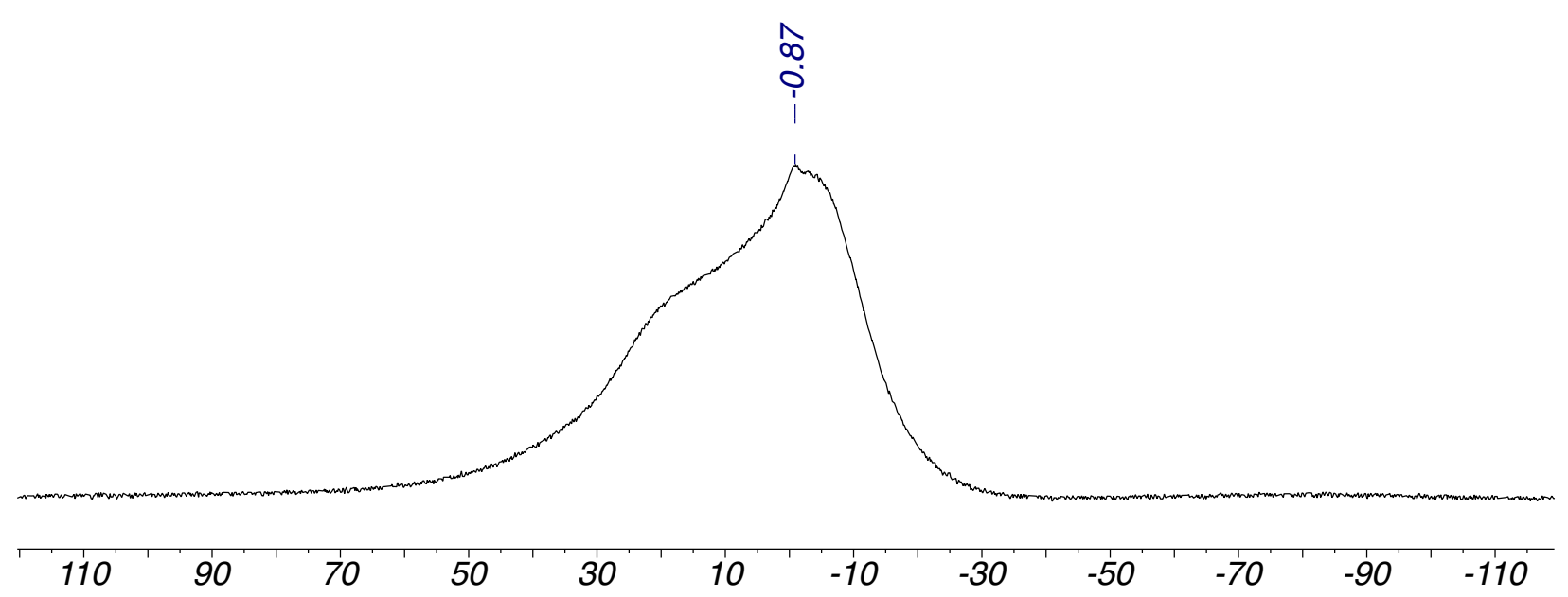

Figure S26 $-{ }^{11} B\left\{{ }^{1} H\right\}$ NMR of 5 in THF-d 8 . 


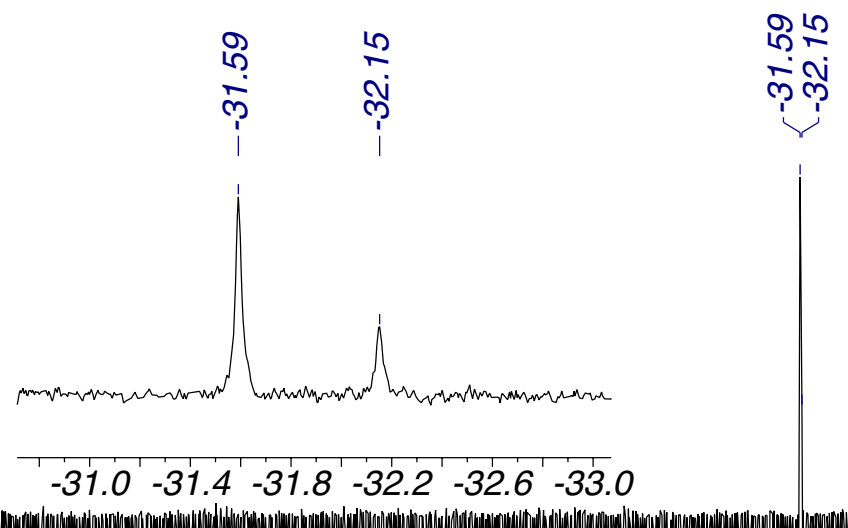

ถุำ

ले लें

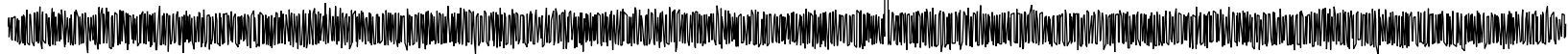

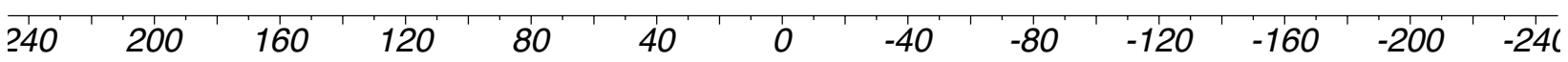
Figure $S 27-{ }^{31} P\left\{{ }^{1} H\right\}$ NMR of 5 in THF-d8.

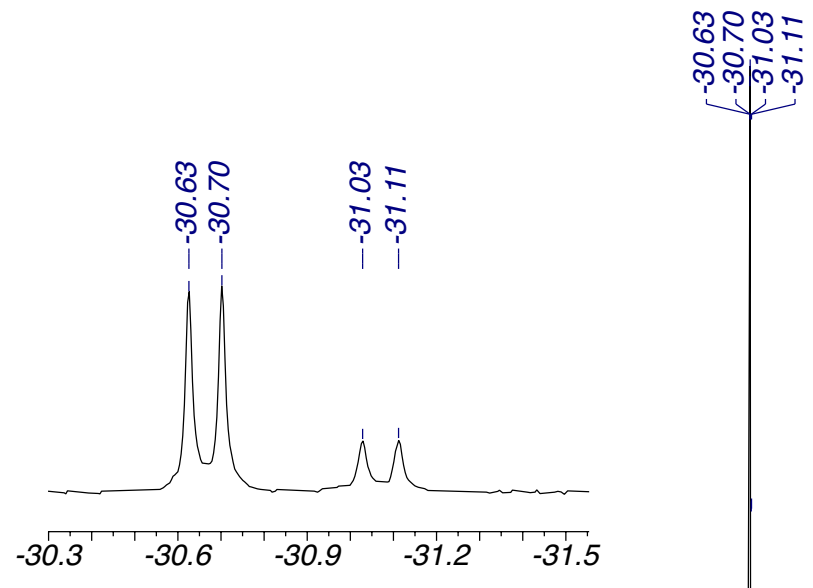

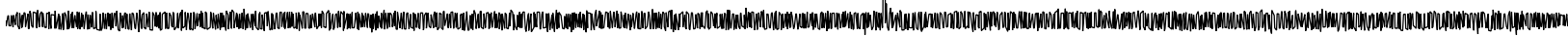
\begin{tabular}{lllllllllllll}
\hline 240 & 200 & 160 & 120 & 80 & 40 & 0 & -40 & -80 & -120 & -160 & -200 & $-24 \mathrm{C}$
\end{tabular} Figure $\mathrm{S} 28-{ }^{31} \mathrm{P}\left\{{ }^{1} \mathrm{H}\right\} \mathrm{NMR}$ of $4+{ }^{13} \mathrm{CO}_{2}$ in $\mathrm{C}_{6} \mathrm{D}_{6}$. 

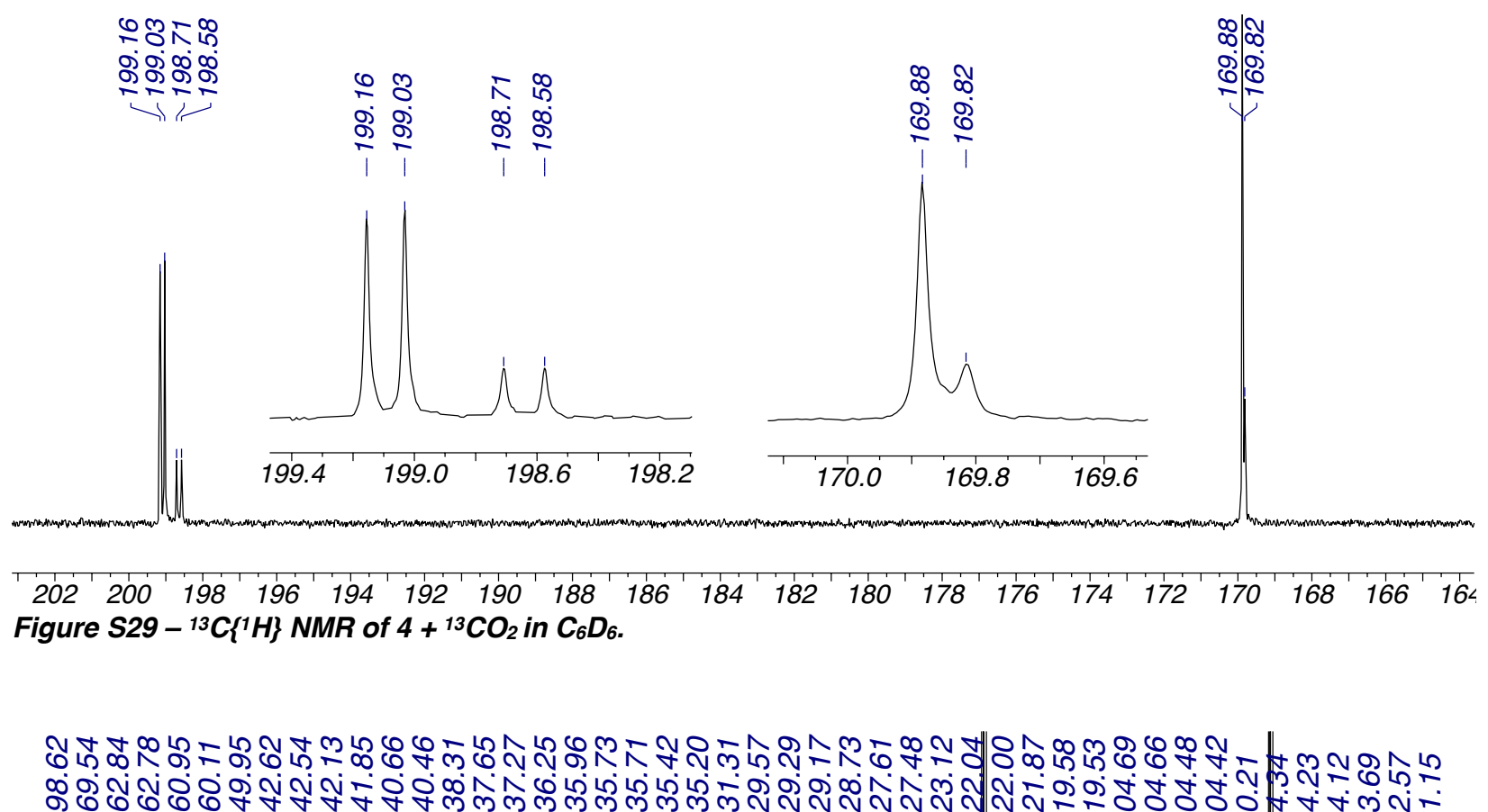
क ర

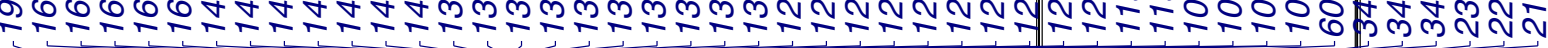

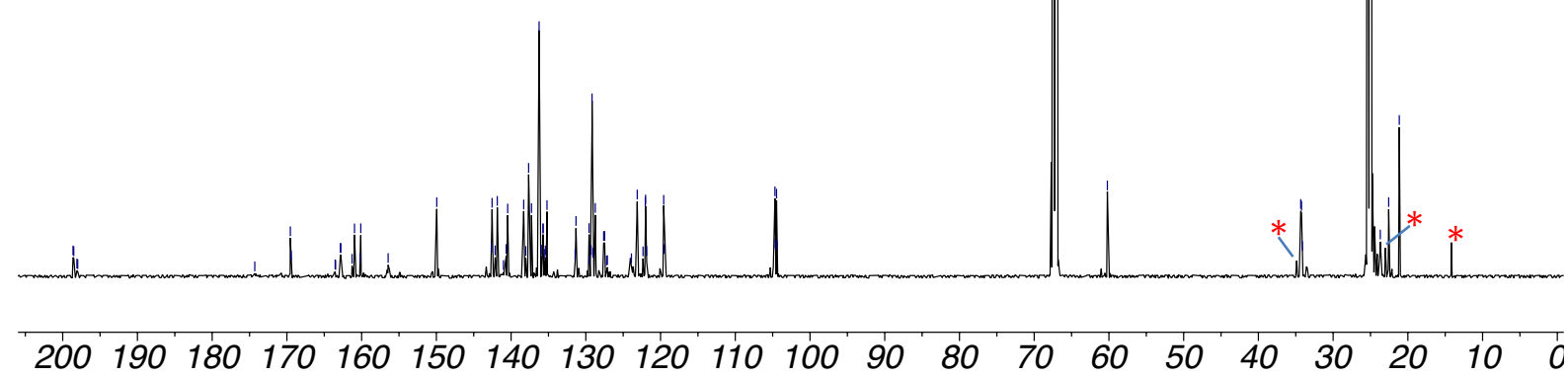

Figure $S 30-{ }^{13} C\left\{{ }^{1} H\right\}$ NMR of 5 in THF-d $d_{8}$. Residual pentane denoted with *. 


\section{IR Spectroscopy Data}

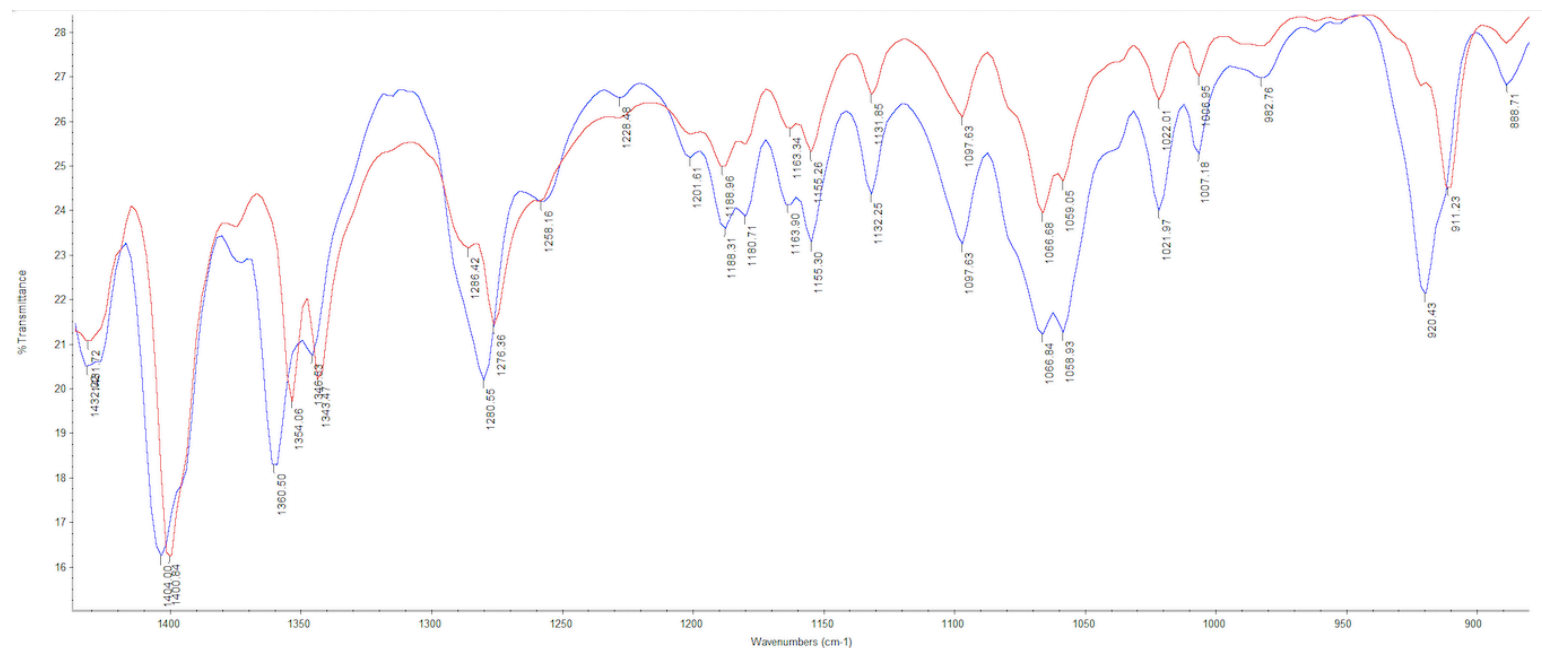

Figure S31 - Overlapping FT-IR spectrum (KBr pellet) of 2-N (blue trace) and $2{ }^{-15} \mathrm{~N}$ (red trace).

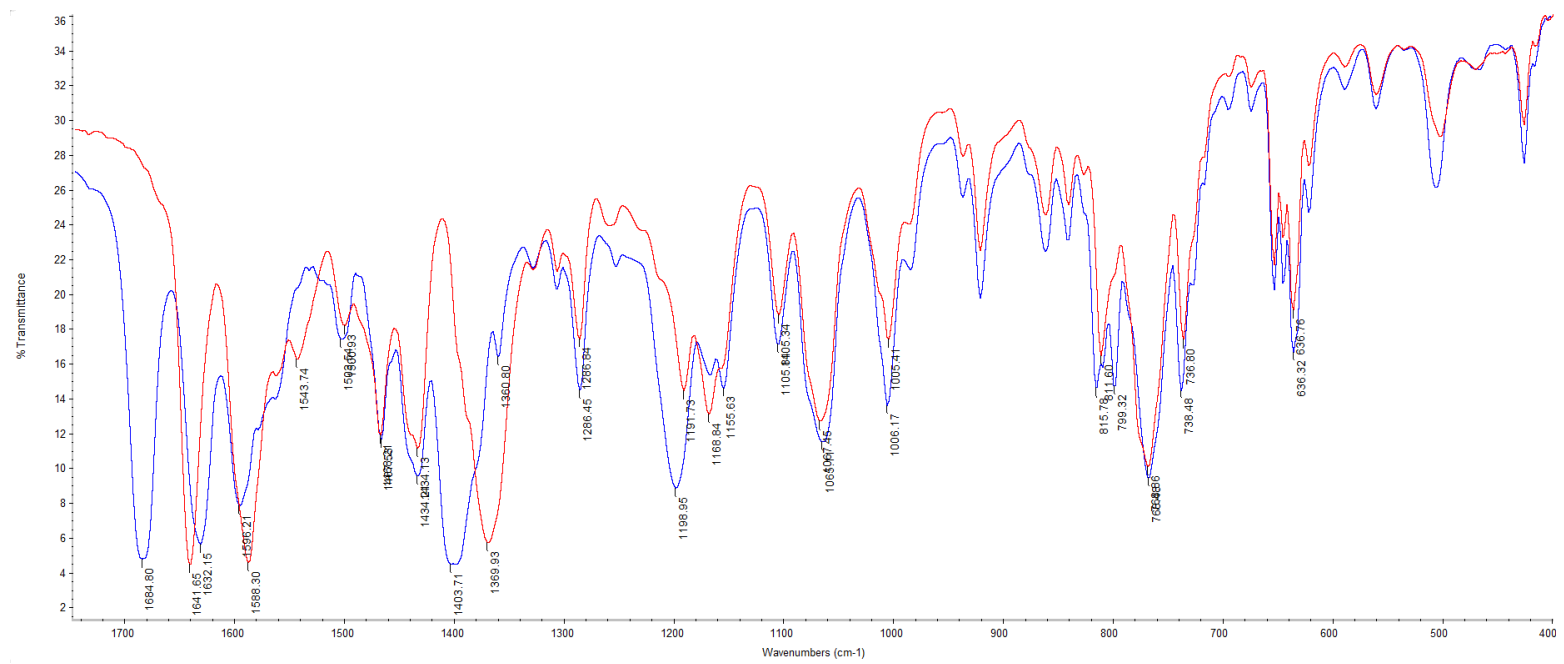

Figure S32 - Overlapping FT-IR spectrum (KBr pellet) of 2-N + $\mathrm{CO}_{2}$ (blue trace) and $2-\mathrm{N}+{ }^{13} \mathrm{CO}_{2}$ (red trace). 


\section{UV-Vis Spectroscopy Data}

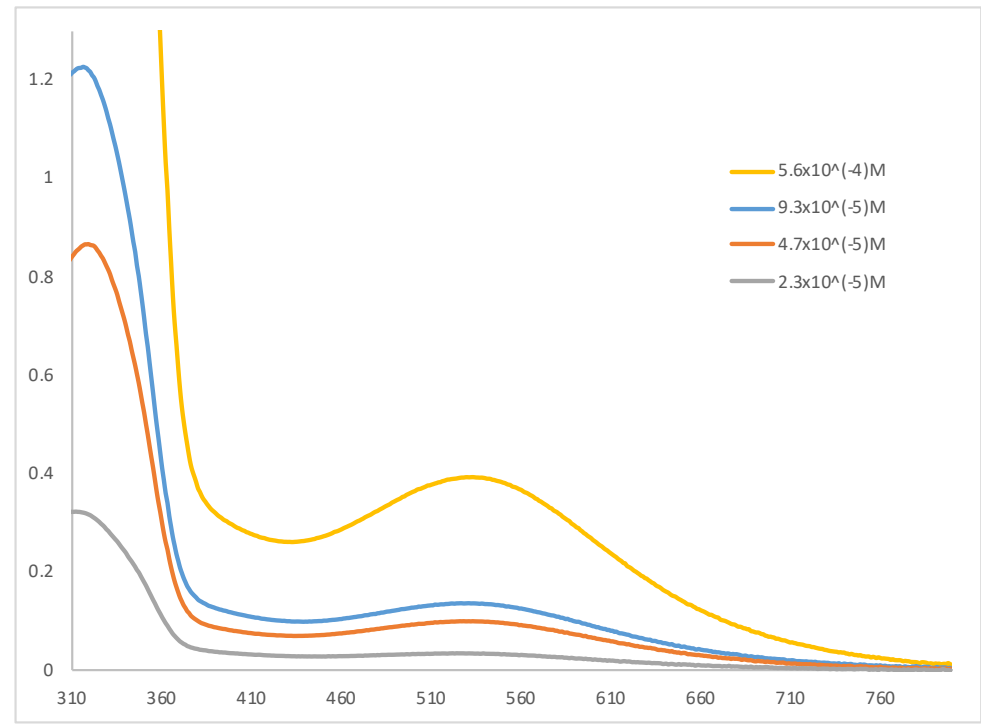

Figure S33 - UV-Vis spectra of 2-N at different concentrations in THF. Epsilon at $\lambda_{318}\left(14,000 \mathrm{M}^{-1} \mathrm{~cm}^{-1}\right)$ was calculated without using the yellow trace. Epsilon at $\lambda_{532}\left(1,200 \mathrm{M}^{-1} \mathrm{~cm}^{-1}\right)$ was calculated using all four traces. 


\section{Crystallographic Data}

Table S2 - Single crystal x-ray diffraction details for complexes BPz2Py3H, 2-N, 4, and 5.

\begin{tabular}{|c|c|c|c|}
\hline Identification code & $\mathrm{BPz}_{2} \mathrm{Py}_{3} \mathrm{H}$ & $2-N$ & 4 \\
\hline Empirical formula & $\mathrm{C}_{30} \mathrm{H}_{28} \mathrm{BN}_{7}$ & $\mathrm{C}_{42} \mathrm{H}_{44} \mathrm{BN}_{8} \mathrm{Sc}$ & $\mathrm{C}_{47} \mathrm{H}_{56} \mathrm{BN}_{7} \mathrm{PSc}$ \\
\hline \begin{tabular}{|l} 
Formula weight \\
\end{tabular} & 497.40 & 716.62 & 805.72 \\
\hline$T(\mathrm{~K})$ & 173.0 & 173 & 173.0 \\
\hline Crystal system & triclinic & monoclinic & triclinic \\
\hline Space group & $\mathrm{P}-1$ & $\mathrm{C} 2 / \mathrm{c}$ & $\mathrm{P}-1$ \\
\hline$a(\mathrm{deg})$ & $9.2828(10)$ & $17.9420(13)$ & $8.3970(18)$ \\
\hline$b$ (deg) & $12.5090(14)$ & $20.4968(17)$ & $10.808(2)$ \\
\hline$c(\mathrm{deg})$ & $12.9773(14)$ & 26.1685(19) & $24.445(5)$ \\
\hline$\alpha(\mathrm{deg})$ & $118.2260(10)$ & 90 & $93.476(6)$ \\
\hline$\beta(\mathrm{deg})$ & $96.3920(10)$ & $93.504(5)$ & $95.859(7)$ \\
\hline$\gamma(\mathrm{deg})$ & $94.6160(10)$ & 90 & $91.758(6)$ \\
\hline$V\left(\AA^{3}\right)$ & $1304.1(2)$ & 9605.6(13) & $2201.4(8)$ \\
\hline$Z$ & 2 & 8 & 2 \\
\hline$\rho_{\text {calc }}\left(\mathrm{g} / \mathrm{cm}^{3}\right)$ & 1.267 & 0.991 & 1.216 \\
\hline$\mu\left(\mathrm{mm}^{-1}\right)$ & 0.078 & 1.560 & 2.080 \\
\hline$F(000)$ & 524.0 & 3024.0 & 856.0 \\
\hline Crystal size $\left(\mathbf{m m}^{3}\right)$ & $0.282 \times 0.259 \times 0.202$ & $\begin{array}{c}0.076 \times 0.054 \times \\
0.039 \\
\end{array}$ & $\begin{array}{c}0.424 \times 0.182 \times \\
0.056 \\
\end{array}$ \\
\hline Radiation & $\operatorname{MoK} \alpha(\lambda=0.71073)$ & $\operatorname{CuK\alpha }(\lambda=1.54178)$ & $\operatorname{CuK\alpha }(\lambda=1.54178)$ \\
\hline $\begin{array}{l}2 \theta \text { range for data collection } \\
\text { (deg) }\end{array}$ & 3.614 to 56.638 & 6.554 to 130.398 & 3.64 to 133.19 \\
\hline Index ranges & $\begin{array}{c}-12 \leq \mathrm{h} \leq 12,-16 \leq \mathrm{k} \leq \\
16,-17 \leq \mathrm{l} \leq 17\end{array}$ & $\begin{array}{l}-21 \leq \mathrm{h} \leq 21,-21 \leq \\
\mathrm{k} \leq 24,-30 \leq \mathrm{l} \leq 22\end{array}$ & $\begin{array}{c}-9 \leq \mathrm{h} \leq 9,-12 \leq \mathrm{k} \leq \\
12,-28 \leq \mathrm{l} \leq 29\end{array}$ \\
\hline Reflections collected & 46401 & 32708 & 29561 \\
\hline Independent reflections & $\begin{array}{c}6500\left[R_{\text {int }}=0.0413\right. \\
\left.R_{\text {sigma }}=0.0257\right]\end{array}$ & $\begin{array}{c}7921\left[\mathrm{R}_{\text {int }}=0.0594\right. \\
\left.\mathrm{R}_{\text {sigma }}=0.0577\right]\end{array}$ & $\begin{array}{c}7754\left[\mathrm{R}_{\text {int }}=0.0294,\right. \\
\left.\mathrm{R}_{\text {sigma }}=0.0246\right]\end{array}$ \\
\hline Data/restraints/parameters & $6500 / 0 / 349$ & $7921 / 0 / 475$ & $7754 / 1123 / 698$ \\
\hline Goodness-of-fit on $\mathrm{F}^{2}$ & 1.019 & 1.058 & 1.046 \\
\hline Final $R$ indexes $[I>=2 \sigma(I)]$ & $\begin{array}{c}\mathrm{R}_{1}=0.0439 \\
\mathrm{w} \mathrm{R}_{2}=0.1032\end{array}$ & $\begin{array}{c}R_{1}=0.0638 \\
w R_{2}=0.1817\end{array}$ & $\begin{array}{c}\mathrm{R}_{1}=0.0359 \\
w \mathrm{R}_{2}=0.0949\end{array}$ \\
\hline Final $R$ indexes [all data] & $\begin{array}{c}\mathrm{R}_{1}=0.0602 \\
\mathrm{wR}_{2}=0.1146\end{array}$ & $\begin{array}{c}\mathrm{R}_{1}=0.0883 \\
\mathrm{wR}_{2}=0.1955\end{array}$ & $\begin{array}{c}\mathrm{R}_{1}=0.0375 \\
\mathrm{wR}_{2}=0.0962\end{array}$ \\
\hline 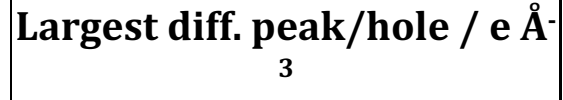 & $0.44 /-0.26$ & $0.57 /-0.49$ & $0.36 /-0.30$ \\
\hline
\end{tabular}


Table S2 continued

\begin{tabular}{|c|c|}
\hline Identification code & 5 \\
\hline Empirical formula & $\mathrm{C}_{50} \mathrm{H}_{50} \mathrm{BN}_{7} \mathrm{O}_{4} \mathrm{PSc}$ \\
\hline Formula weight & 899.71 \\
\hline$T(\mathrm{~K})$ & 173.0 \\
\hline Crystal system & triclinic \\
\hline Space group & P-1 \\
\hline$a(\mathrm{deg})$ & $8.7025(3)$ \\
\hline$b(\mathrm{deg})$ & $12.7011(4)$ \\
\hline$c(\mathrm{deg})$ & $21.2188(10)$ \\
\hline$\alpha(\operatorname{deg})$ & $90.965(3)$ \\
\hline$\beta(\operatorname{deg})$ & $91.117(3)$ \\
\hline$\gamma(\mathrm{deg})$ & 101.418(2) \\
\hline$V\left(\AA^{3}\right)$ & $2297.98(15)$ \\
\hline$Z$ & 2 \\
\hline$\rho_{\text {calc }}\left(\mathrm{g} / \mathrm{cm}^{3}\right)$ & 1.300 \\
\hline$\mu\left(\mathrm{mm}^{-1}\right)$ & 2.122 \\
\hline$F(000)$ & 944.0 \\
\hline Crystal size $\left(\mathrm{mm}^{3}\right)$ & $\begin{array}{c}0.221 \times 0.065 \times \\
0.059\end{array}$ \\
\hline Radiation & $\begin{array}{l}\mathrm{CuK \alpha}(\lambda= \\
1.54178)\end{array}$ \\
\hline $\begin{array}{l}2 \theta \text { range for data collection } \\
\text { (deg) }\end{array}$ & 4.166 to 133.188 \\
\hline Index ranges & $\begin{array}{l}-10 \leq h \leq 10,-15 \leq \\
k \leq 13,-23 \leq 1 \leq 25\end{array}$ \\
\hline Reflections collected & 26336 \\
\hline Independent reflections & $\begin{array}{c}7812\left[\mathrm{R}_{\text {int }}=\right. \\
0.0621, \mathrm{R}_{\text {sigma }}= \\
0.0718]\end{array}$ \\
\hline Data/restraints/parameters & $7812 / 1276 / 726$ \\
\hline \begin{tabular}{|l|} 
Goodness-of-fit on $\mathbf{F}^{2}$ \\
\end{tabular} & 1.058 \\
\hline Final $R$ indexes $[\mathrm{I}>=2 \sigma(\mathrm{I})]$ & $\begin{array}{c}\mathrm{R}_{1}=0.0528 \\
\mathrm{wR} \mathrm{R}_{2}=0.1284\end{array}$ \\
\hline Final $R$ indexes [all data] & $\begin{array}{c}\mathrm{R}_{1}=0.0652 \\
\mathrm{wR}_{2}=0.1355\end{array}$ \\
\hline 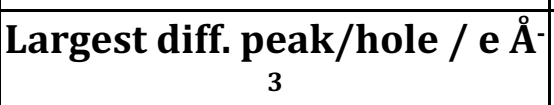 & $0.54 /-0.36$ \\
\hline
\end{tabular}




\section{References}

(1) Pangborn, A. B.; Giardello, M. A.; Grubbs, R. H.; Rosen, R. K.; Timmers, F. J. Organometallics 1996, 15, 1518.

(2) Doszczak, L.; Kraft, P.; Weber, H.-P.; Bertermann, R.; Triller, A.; Hatt, H.;

Tacke, R. Angewandte Chemie International Edition 2007, 46, 3367.

(3) Ünal, E. A.; Wiedemann, D.; Seiffert, J.; Boyd, J. P.; Grohmann, A. Tetrahedron Letters 2012, 53, 54.

(4) Saeed, I.; Katao, S.; Nomura, K. Inorganic Chemistry 2009, 48, 5011.

(5) Emslie, D. J. H.; Piers, W. E.; Parvez, M.; McDonald, R. Organometallics 2002, $21,4226$.

(6) Green, R. A.; Hartwig, J. F. Organic Letters 2014, 16, 4388.

(7) Boeré, R. T.; Masuda, J. D. Canadian Journal of Chemistry 2002, 80, 1607.

(8) Fulmer, G. R.; Miller, A. J. M.; Sherden, N. H.; Gottlieb, H. E.; Nudelman, A.;

Stoltz, B. M.; Bercaw, J. E.; Goldberg, K. I. Organometallics 2010, 29, 2176.

(9) Bruker-AXS. SAINT. Madison, Wisconsin, USA. 2017.

(10) Bruker-AXS. XPREP. Madison, Wisconsin, USA. 2017.

(11) Dolomanov, O. V.; Bourhis, L. J.; Gildea, R. J.; Howard, J. A. K.; Puschmann, H. Journal of Applied Crystallography 2009, 42, 339.

(12) Sheldrick, G. M. Acta Crystallographica a-Foundation and Advances 2015, 71, 3.

(13) Sheldrick, G. M. Acta Crystallographica Section C-Structural Chemistry 2015, $71,3$.

(14) Spek, A. L. Acta Crystallographica Section C-Structural Chemistry 2015, 71, 9. (15) Barnard, J. H.; Yruegas, S.; Couchman, S. A.; Wilson, D. J. D.; Dutton, J. L.; Martin, C. D. Organometallics 2016, 35, 929.

(16) Morris, G. A., Diffusion-Ordered Spectroscopy. In eMagRes.

(17) Matsuo, S.; Makita, T. International Journal of Thermophysics 1993, 14, 67.

(18) Schulze, B. M.; Watkins, D. L.; Zhang, J.; Ghiviriga, I.; Castellano, R. K. Organic \& Biomolecular Chemistry 2014, 12, 7932.

(19) Beh, D. W.; Piers, W. E.; del Rosal, I.; Maron, L.; Gelfand, B. S.; Gendy, C.; Lin, J.B. Dalton Transactions 2018, 47, 13680. 


\section{Computational Details}

Calculations were carried out with Gaussian09' at the DFT level, with the hybrid functional B3PW91." Scandium and phosphorus atoms were treated with smallcore pseudopotentials from the Stuttgart group, with additional polarization orbitals.iii The other atoms that were part of the systems (boron, nitrogen, carbon, and hydrogen) were treated with the extended all electron Gaussian-Type 6-31G** Pople basis set. ${ }^{i v}$ No symmetry constraints were considered for the geometry optimizations that took as starting point the experimentally obtained geometries of both reagents and products. Analytical calculations of the vibrational frequencies confirmed that the structures obtained were the critical points involved in the reactive process, and also obtained the thermal corrections over the energies. Transition states obtained where connected with its respective intermediates with Intrinsic Reaction Coordinate (IRC) calculations. Bonding was studied doing Natural Bond Orbital analysis over the optimized structures, with NBO software. ${ }^{v}$
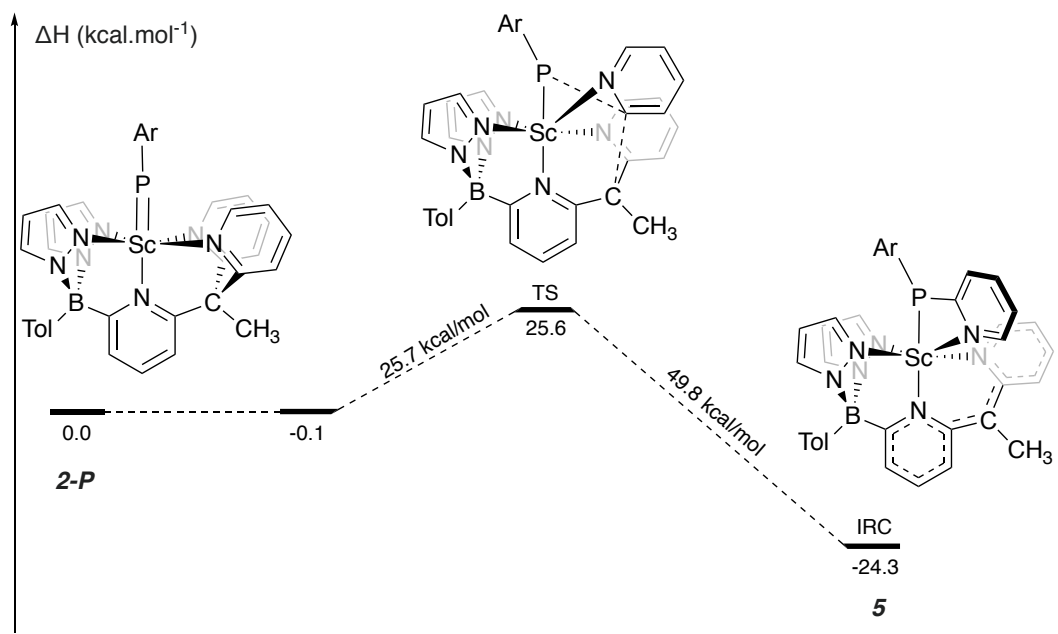

Figure S34 - Computed enthalpy profile at room temperature for the formation of 5 from the putative 2-P 


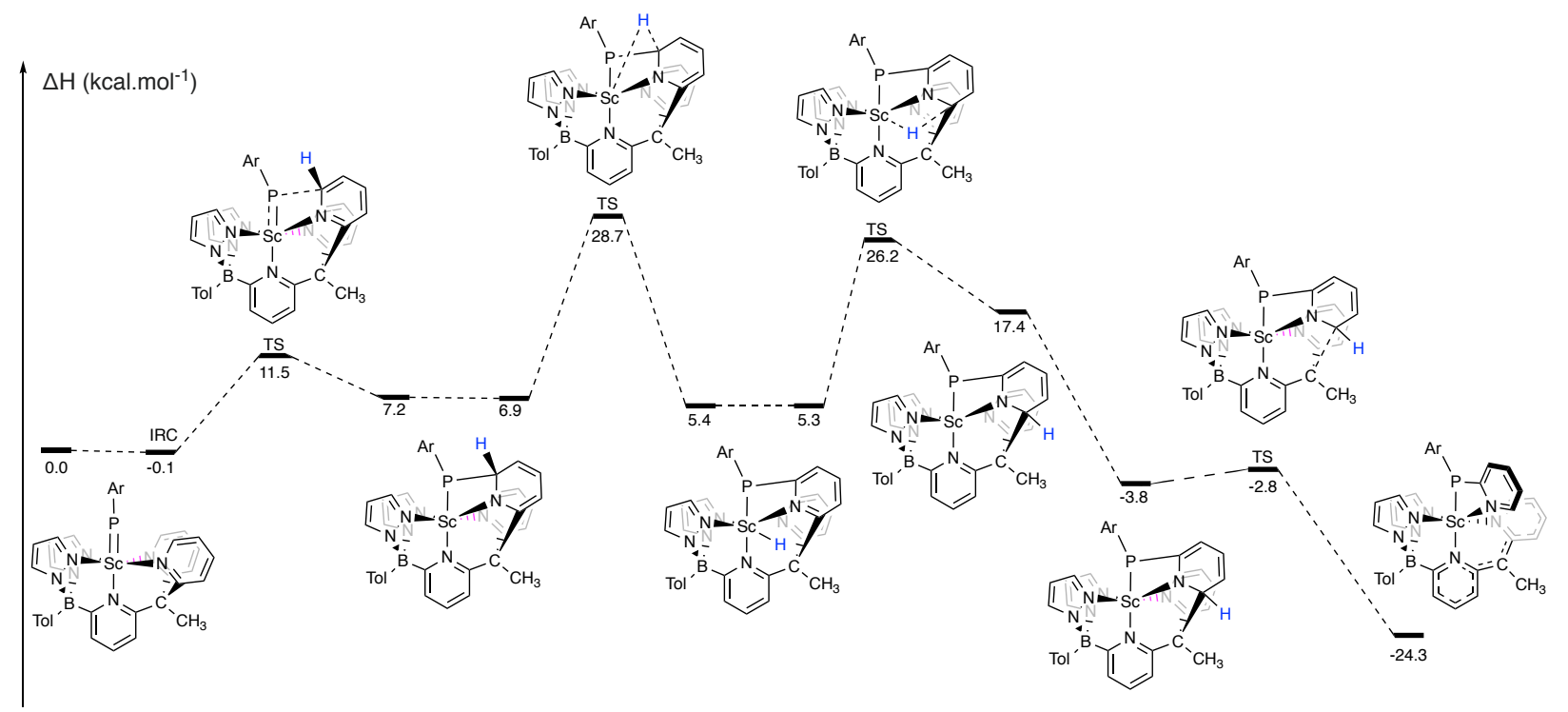

Figure S35 - Computed enthalpy profile at room temperature of an alternative pathway for the formation of 5 from the putative 2-P

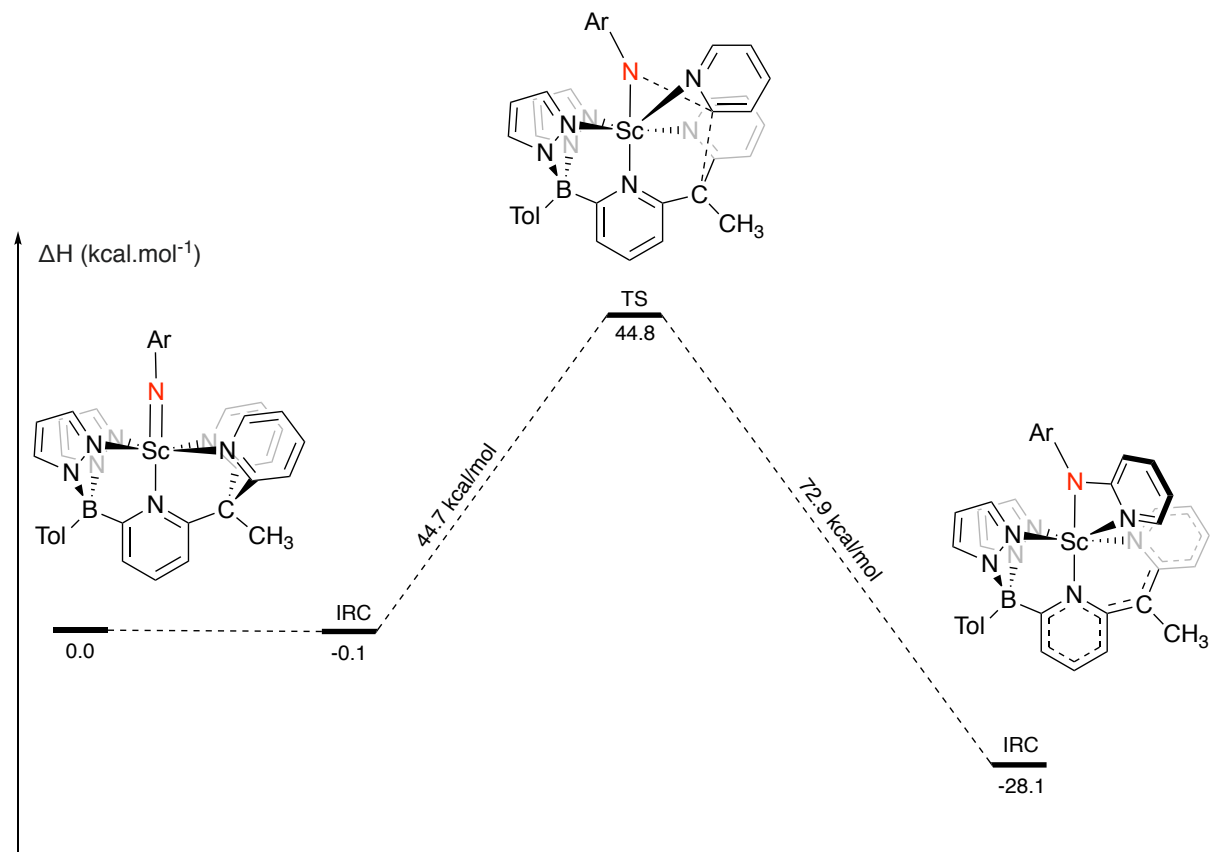

Figure S36 - Computed enthalpy profile at room temperature for the formation of putative 5-N from the putative 2-N 


\section{Computational references}

(i) Gaussian 09, Revision A.02, Frisch, M. J.; Trucks, G. W.; Schlegel, H. B.; Scuseria, G. E.; Robb, M. A.; Cheeseman, J. R.; Scalmani, G.; Barone,V.; Mennucci, B.; Petersson, G. A.; Nakatsuji, H.; Caricato, M.; Li, X.; Hratchian, H. P.; Izmaylov, A. F.; Bloino, J.; Zheng, G.; Sonnenberg, J. L.; Hada, M.; Ehara, M.; Toyota, K.; Fukuda, R.; Hasegawa, J.; Ishida, M.; Nakajima, T.; Honda, Y.; Kitao, O.; Nakai, H.; Vreven, T.; Montgomery, J. A.; Peralta, Jr., J. E.; Ogliaro, F.; Bearpark, M.; Heyd, J. J.; Brothers, E.; Kudin, K. N.; Staroverov,V. N.; Kobayashi, R.; Normand, J.; Raghavachari, K.; Rendell, A.; Burant, J. C.; lyengar, S. S.; Tomasi, J.; Cossi, M.; Rega, N.; Millam, J. M.; Klene, M.; Knox, J. E.; Cross, J. B.; Bakken, V.; Adamo, C.; Jaramillo, J.; Gomperts, R.; Stratmann, R. E.; Yazyev, O.; Austin, A. J.; Cammi, R.; Pomelli, C.; Ochterski, J. W.; Martin, R. L.; Morokuma, K.; Zakrzewski, V.; Voth,G.G.A.; Salvador, P.; Dannenberg, J. J.; Dapprich,S.; Daniels, A. D.; Farkas, Ö.; Foresman, J. B.; Ortiz, J. V.; Cioslowski, J.; Fox, D. J. Gaussian, Inc., Wallingford CT, 2009.

(ii) (a) Becke, A. D. J. Chem. Phys., 1993, 98, 5648. (b) Perdew, J. P.; Wang, Y. Phys. Rev. B, 1992, 45, 13244. (c) Burke, K.; Perdew, J. P.; Yang, W. in Electronic Density Functional Theory: Recent Progress and New Directions, Eds. Dobson, J. F.; Vignale, G.; Das, M. P.; Plenum, New York, 1998.

(iii) (a) Bergner, A.; Dolg, M.; Kuechle, W.; Stoll, H.; Preuss, H. Mol. Phys. 1993, 80, 1431. (b) Leininger, T.; Berning, A.; Nicklass, A.; Stoll, H.; Werner, H. J.; Flad, H. J. Chem. Phys. 1997, 19, 217.

(iv) Ditchfield, R.; Hehre, W. J.; Pople, J. A. J. Chem. Phys., 1971, 54, 724.

(v) NBO Version 3.1, Glendening, E. D.; Reed, A. E.; Carpenter, J. E.; Weinhold, $\mathrm{F}$. 
Cartesian Coordinates of Optimized Structures

\begin{tabular}{|c|c|c|c|}
\hline $\mathrm{N}$ & 9.21954800 & 2.49332400 & 11.17588900 \\
\hline $\mathrm{N}$ & 8.47075400 & 2.43992200 & 12.30243900 \\
\hline $\mathrm{N}$ & 8.26793200 & 5.23285700 & 11.18886700 \\
\hline $\mathrm{N}$ & 7.65341600 & 4.87209600 & 12.34017800 \\
\hline $\mathrm{N}$ & 6.40449800 & 3.04112800 & 10.09074500 \\
\hline $\mathrm{N}$ & 7.26962600 & 4.74588200 & 7.81016000 \\
\hline $\mathrm{N}$ & 8.21753600 & 1.99229400 & 8.07137000 \\
\hline $\mathrm{C}$ & 10.27089600 & 1.68691400 & 11.35586500 \\
\hline $\mathrm{C}$ & 10.20243900 & 1.07210100 & 12.60752300 \\
\hline $\mathrm{C}$ & 9.04827400 & 1.58487800 & 13.17663600 \\
\hline $\mathrm{C}$ & 8.41259200 & 6.56332400 & 11.21974100 \\
\hline $\mathrm{C}$ & 7.84954100 & 7.08843600 & 12.38559500 \\
\hline $\mathrm{C}$ & 7.37294600 & 5.97643000 & 13.06378700 \\
\hline $\mathrm{C}$ & 6.74128800 & 3.06435600 & 14.06983300 \\
\hline $\mathrm{C}$ & 5.81525400 & 2.04987500 & 14.37033000 \\
\hline $\mathrm{C}$ & 5.49704700 & 1.69217800 & 15.67958700 \\
\hline $\mathrm{C}$ & 6.09863100 & 2.32899000 & 16.76920200 \\
\hline $\mathrm{C}$ & 7.05308400 & 3.31244100 & 16.49117100 \\
\hline $\mathrm{C}$ & 7.36937300 & 3.65991500 & 15.17911200 \\
\hline $\mathrm{C}$ & 5.72920400 & 1.97484700 & 18.18506100 \\
\hline $\mathrm{C}$ & 6.06235800 & 3.17721100 & 11.39965400 \\
\hline $\mathrm{C}$ & 4.71106000 & 3.30721300 & 11.75186100 \\
\hline $\mathrm{C}$ & 3.72466600 & 3.23246700 & 10.78424600 \\
\hline $\mathrm{C}$ & 4.09522200 & 3.00387000 & 9.46539800 \\
\hline $\mathrm{C}$ & 5.45114700 & 2.91727200 & 9.14600800 \\
\hline C & 5.88283300 & 2.70180300 & 7.67236800 \\
\hline $\mathrm{C}$ & 4.67949700 & 2.20675900 & 6.85106200 \\
\hline $\mathrm{C}$ & 7.65787000 & 5.96052200 & 7.38624900 \\
\hline $\mathrm{C}$ & 7.17455200 & 6.54847400 & 6.22659500 \\
\hline $\mathrm{C}$ & 6.28374600 & 5.81547100 & 5.44874900 \\
\hline $\mathrm{C}$ & 5.87552700 & 4.56228400 & 5.88708600 \\
\hline $\mathrm{C}$ & 6.36431200 & 4.05493300 & 7.09920100 \\
\hline $\mathrm{C}$ & 9.24211500 & 1.12984500 & 7.96579000 \\
\hline $\mathrm{C}$ & 9.11245800 & -0.13883800 & 7.42126900 \\
\hline $\mathrm{C}$ & 7.86273400 & -0.51621400 & 6.94226600 \\
\hline $\mathrm{C}$ & 6.81001300 & 0.38934700 & 7.01520800 \\
\hline
\end{tabular}




\begin{tabular}{|c|c|c|c|}
\hline $\mathrm{C}$ & 7.01099200 & 1.65095200 & 7.58825200 \\
\hline B & 7.21990900 & 3.36618400 & 12.54668500 \\
\hline $\mathrm{H}$ & 11.03091500 & 1.62792000 & 10.58938000 \\
\hline $\mathrm{H}$ & 10.89785400 & 0.37329900 & 13.04752900 \\
\hline $\mathrm{H}$ & 8.61151400 & 1.40850800 & 14.14642300 \\
\hline $\mathrm{H}$ & 8.94044500 & 7.05785400 & 10.41607900 \\
\hline $\mathrm{H}$ & 7.80909600 & 8.11977900 & 12.70246800 \\
\hline $\mathrm{H}$ & 6.85829700 & 5.90229400 & 14.00910100 \\
\hline $\mathrm{H}$ & 5.33869500 & 1.50202200 & 13.56065700 \\
\hline $\mathrm{H}$ & 4.77415600 & 0.89801100 & 15.85727800 \\
\hline $\mathrm{H}$ & 7.56791200 & 3.80521800 & 17.31382000 \\
\hline $\mathrm{H}$ & 8.15409200 & 4.39526500 & 15.02049100 \\
\hline $\mathrm{H}$ & 4.89130100 & 2.58780100 & 18.54037900 \\
\hline $\mathrm{H}$ & 5.42316000 & 0.92762700 & 18.27016500 \\
\hline $\mathrm{H}$ & 6.56532200 & 2.14095500 & 18.87114600 \\
\hline $\mathrm{H}$ & 4.45042700 & 3.47825000 & 12.78952000 \\
\hline $\mathrm{H}$ & 2.67523200 & 3.33967600 & 11.04662800 \\
\hline $\mathrm{H}$ & 3.32978000 & 2.91562000 & 8.70710600 \\
\hline $\mathrm{H}$ & 3.91296000 & 2.97866100 & 6.76684600 \\
\hline $\mathrm{H}$ & 4.22299600 & 1.32999300 & 7.31589500 \\
\hline $\mathrm{H}$ & 4.98459600 & 1.93494800 & 5.83811100 \\
\hline $\mathrm{H}$ & 8.42088700 & 6.43943300 & 7.99152700 \\
\hline $\mathrm{H}$ & 7.51781800 & 7.53407800 & 5.93231100 \\
\hline $\mathrm{H}$ & 5.90637100 & 6.21214100 & 4.51033000 \\
\hline $\mathrm{H}$ & 5.18020800 & 3.99347900 & 5.28463000 \\
\hline $\mathrm{H}$ & 10.19588400 & 1.51585800 & 8.31667300 \\
\hline $\mathrm{H}$ & 9.97177800 & -0.79815300 & 7.36624700 \\
\hline $\mathrm{H}$ & 7.70609300 & -1.49665600 & 6.50145600 \\
\hline $\mathrm{H}$ & 5.84199200 & 0.10055200 & 6.62903100 \\
\hline Sc & 8.63393900 & 3.79106500 & 9.46506200 \\
\hline $\mathrm{P}$ & 10.64378800 & 4.50247900 & 8.58251700 \\
\hline $\mathrm{C}$ & 12.06807200 & 5.07438000 & 7.58986200 \\
\hline $\mathrm{C}$ & 12.94923800 & 6.06868600 & 8.10379500 \\
\hline $\mathrm{C}$ & 12.33034600 & 4.54163500 & 6.29549300 \\
\hline $\mathrm{C}$ & 14.03400800 & 6.49717100 & 7.33518300 \\
\hline $\mathrm{C}$ & 13.42894600 & 5.00051200 & 5.56530000 \\
\hline $\mathrm{C}$ & 14.28346700 & 5.97395300 & 6.07111300 \\
\hline $\mathrm{H}$ & 14.70099400 & 7.25719400 & 7.73773600 \\
\hline $\mathrm{H}$ & 13.62097000 & 4.58408600 & 4.57805500 \\
\hline $\mathrm{H}$ & 15.13422200 & 6.31879900 & 5.48869000 \\
\hline $\mathrm{C}$ & 12.74744000 & 6.68349600 & 9.47993500 \\
\hline $\mathrm{H}$ & 11.86125800 & 6.19773900 & 9.90936200 \\
\hline $\mathrm{C}$ & 11.45083300 & 3.46566300 & 5.67873800 \\
\hline $\mathrm{H}$ & 10.63654800 & 3.29068600 & 6.39454700 \\
\hline $\mathrm{C}$ & 10.81652800 & 3.92188400 & 4.35912800 \\
\hline $\mathrm{H}$ & 10.23000500 & 4.83481500 & 4.50331000 \\
\hline
\end{tabular}




$\begin{array}{lccc}\mathrm{H} & 11.57280200 & 4.13067100 & 3.59321300 \\ \mathrm{H} & 10.14963500 & 3.14755600 & 3.96008700 \\ \mathrm{C} & 12.21669300 & 2.14836900 & 5.49870200 \\ \mathrm{H} & 12.63869200 & 1.80764500 & 6.45008800 \\ \mathrm{H} & 11.55705100 & 1.36130700 & 5.11235000 \\ \mathrm{H} & 13.04760600 & 2.25890500 & 4.79189200 \\ \mathrm{C} & 13.92888700 & 6.39588000 & 10.41439900 \\ \mathrm{H} & 14.10300600 & 5.31892800 & 10.50205500 \\ \mathrm{H} & 14.85601200 & 6.85619800 & 10.05236400 \\ \mathrm{H} & 13.73330200 & 6.79085100 & 11.41838500 \\ \mathrm{C} & 12.45876300 & 8.18761500 & 9.39496000 \\ \mathrm{H} & 11.58996100 & 8.38516100 & 8.75784700 \\ \mathrm{H} & 12.25449900 & 8.60086100 & 10.39011800 \\ \mathrm{H} & 13.30745000 & 8.74117600 & 8.97579100\end{array}$

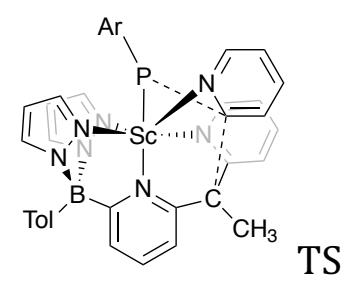

$\begin{array}{cccc}\mathrm{N} & 9.12906000 & 1.71547300 & 11.18893500 \\ \mathrm{~N} & 8.49091900 & 2.26839700 & 12.24627900 \\ \mathrm{~N} & 9.34053900 & 4.42781500 & 10.52425900 \\ \mathrm{~N} & 8.45951700 & 4.69310900 & 11.52173400 \\ \mathrm{~N} & 6.68975800 & 2.98254800 & 9.74598900 \\ \mathrm{~N} & 8.10238100 & 4.06586200 & 7.56084300 \\ \mathrm{~N} & 7.72959200 & 0.77399700 & 8.79692900 \\ \mathrm{C} & 9.88603800 & 0.71388300 & 11.64973300 \\ \mathrm{C} & 9.74546200 & 0.60327900 & 13.03493400 \\ \mathrm{C} & 8.85187200 & 1.60835400 & 13.36977400 \\ \mathrm{C} & 9.98470000 & 5.57558400 & 10.26723400 \\ \mathrm{C} & 9.49231600 & 6.61030200 & 11.06273200 \\ \mathrm{C} & 8.51184700 & 6.00279100 & 11.83361100 \\ \mathrm{C} & 7.05403700 & 3.84923200 & 13.63922700 \\ \mathrm{C} & 5.99365500 & 3.11319100 & 14.20319500 \\ \mathrm{C} & 5.61394700 & 3.24639200 & 15.53692500 \\ \mathrm{C} & 6.28576600 & 4.12181800 & 16.39613000 \\ \mathrm{C} & 7.37461400 & 4.82201700 & 15.87031300 \\ \mathrm{C} & 7.75078100 & 4.67904500 & 14.53508200 \\ \mathrm{C} & 5.85280500 & 4.30180900 & 17.82643100 \\ \mathrm{C} & 6.34351700 & 3.32384100 & 11.01362400 \\ \mathrm{C} & 4.98768000 & 3.53532600 & 11.28525200 \\ \mathrm{C} & 4.03119000 & 3.28552000 & 10.30737200 \\ \mathrm{C} & 4.42570900 & 2.85103700 & 9.04809300 \\ \mathrm{C} & 5.78948500 & 2.74258500 & 8.77170000\end{array}$




\begin{tabular}{|c|c|c|c|}
\hline $\mathrm{C}$ & 6.33745000 & 2.40108800 & 7.37481800 \\
\hline $\mathrm{C}$ & 5.18356300 & 2.00624900 & 6.44464200 \\
\hline $\mathrm{C}$ & 8.73746200 & 5.18616300 & 7.18050700 \\
\hline $\mathrm{C}$ & 8.31967300 & 5.97901400 & 6.12317200 \\
\hline $\mathrm{C}$ & 7.18701400 & 5.58827300 & 5.41937600 \\
\hline $\mathrm{C}$ & 6.53261700 & 4.42036700 & 5.79835700 \\
\hline $\mathrm{C}$ & 7.00618900 & 3.67143300 & 6.87796400 \\
\hline $\mathrm{C}$ & 7.61933900 & -0.48041000 & 9.28394000 \\
\hline $\mathrm{C}$ & 7.18261500 & -1.54377600 & 8.53944700 \\
\hline $\mathrm{C}$ & 6.90041700 & -1.29657700 & 7.16601400 \\
\hline $\mathrm{C}$ & 6.99034000 & -0.04008400 & 6.64901900 \\
\hline $\mathrm{C}$ & 7.38692300 & 1.09565900 & 7.48010600 \\
\hline B & 7.55022200 & 3.54321700 & 12.12188600 \\
\hline $\mathrm{H}$ & 10.48160800 & 0.12787300 & 10.96179300 \\
\hline $\mathrm{H}$ & 10.21925500 & -0.10307900 & 13.69974100 \\
\hline $\mathrm{H}$ & 8.45153100 & 1.89312600 & 14.32942800 \\
\hline $\mathrm{H}$ & 10.78317500 & 5.59743400 & 9.53862700 \\
\hline $\mathrm{H}$ & 9.80489300 & 7.64336100 & 11.08683900 \\
\hline $\mathrm{H}$ & 7.85684500 & 6.41798100 & 12.58368400 \\
\hline $\mathrm{H}$ & 5.46419600 & 2.38415300 & 13.59514100 \\
\hline $\mathrm{H}$ & 4.78694900 & 2.65089900 & 15.91908000 \\
\hline $\mathrm{H}$ & 7.94947800 & 5.48095000 & 16.51833500 \\
\hline $\mathrm{H}$ & 8.63810700 & 5.20990900 & 14.20270800 \\
\hline $\mathrm{H}$ & 5.08922400 & 5.08507300 & 17.91198600 \\
\hline $\mathrm{H}$ & 5.42021100 & 3.38279000 & 18.23368400 \\
\hline $\mathrm{H}$ & 6.69104400 & 4.59496200 & 18.46564200 \\
\hline $\mathrm{H}$ & 4.68482900 & 3.88853000 & 12.26303500 \\
\hline $\mathrm{H}$ & 2.97555500 & 3.42689100 & 10.52483400 \\
\hline $\mathrm{H}$ & 3.68083500 & 2.62916900 & 8.29559000 \\
\hline $\mathrm{H}$ & 4.42379700 & 2.79053600 & 6.39097800 \\
\hline $\mathrm{H}$ & 4.71175100 & 1.08467500 & 6.79099900 \\
\hline $\mathrm{H}$ & 5.54590100 & 1.83327000 & 5.42989800 \\
\hline $\mathrm{H}$ & 9.61532500 & 5.45620700 & 7.75235400 \\
\hline $\mathrm{H}$ & 8.87832400 & 6.87088600 & 5.86190800 \\
\hline $\mathrm{H}$ & 6.81806700 & 6.17558800 & 4.58372100 \\
\hline $\mathrm{H}$ & 5.65542200 & 4.09924800 & 5.25246200 \\
\hline $\mathrm{H}$ & 7.91334600 & -0.59319900 & 10.32471200 \\
\hline $\mathrm{H}$ & 7.09861000 & -2.53281700 & 8.97424000 \\
\hline $\mathrm{H}$ & 6.62571000 & -2.12122300 & 6.51221600 \\
\hline $\mathrm{H}$ & 6.82066500 & 0.11492700 & 5.58966800 \\
\hline Sc & 8.78084000 & 2.58291300 & 9.17662100 \\
\hline $\mathrm{P}$ & 9.62942700 & 1.52402300 & 7.01190200 \\
\hline $\mathrm{C}$ & 10.41853500 & 2.71593700 & 5.81333800 \\
\hline $\mathrm{C}$ & 11.55967700 & 3.48902300 & 6.18057500 \\
\hline C & 9.97717200 & 2.77147800 & 4.46131700 \\
\hline $\mathrm{C}$ & 12.19036300 & 4.29720800 & 5.22774100 \\
\hline
\end{tabular}




$\begin{array}{lccc}\mathrm{C} & 10.62868800 & 3.60271900 & 3.54682500 \\ \mathrm{C} & 11.72661800 & 4.36910800 & 3.92050800 \\ \mathrm{H} & 13.06189300 & 4.88126200 & 5.50921900 \\ \mathrm{H} & 10.28162100 & 3.64378400 & 2.51801500 \\ \mathrm{H} & 12.22475800 & 5.00778400 & 3.19538100 \\ \mathrm{C} & 12.16120800 & 3.39522500 & 7.57580200 \\ \mathrm{H} & 11.31504200 & 3.28431800 & 8.27644400 \\ \mathrm{C} & 8.85626100 & 1.86719400 & 3.96421800 \\ \mathrm{H} & 8.20312500 & 1.67342600 & 4.82196100 \\ \mathrm{C} & 7.99504200 & 2.47576800 & 2.85303100 \\ \mathrm{H} & 7.62683000 & 3.47028400 & 3.12365000 \\ \mathrm{H} & 8.54338500 & 2.56626800 & 1.90854600 \\ \mathrm{H} & 7.13042600 & 1.83247500 & 2.65283600 \\ \mathrm{C} & 9.43428600 & 0.51515700 & 3.51702600 \\ \mathrm{H} & 9.97204000 & 0.03487800 & 4.33915500 \\ \mathrm{H} & 8.63810800 & -0.16250600 & 3.18633600 \\ \mathrm{H} & 10.12984200 & 0.65340400 & 2.68115600 \\ \mathrm{C} & 13.01659400 & 2.12690900 & 7.72291600 \\ \mathrm{H} & 12.43220300 & 1.23017100 & 7.49791700 \\ \mathrm{H} & 13.86666200 & 2.16290300 & 7.03216400 \\ \mathrm{H} & 13.40940400 & 2.03495200 & 8.74263100 \\ \mathrm{C} & 12.96840400 & 4.62233500 & 8.01054500 \\ \mathrm{H} & 12.42822800 & 5.55942800 & 7.83279700 \\ \mathrm{H} & 13.20753600 & 4.55765900 & 9.07758800 \\ \mathrm{H} & 13.92235000 & 4.69073900 & 7.47608600\end{array}$

\begin{tabular}{|c|c|c|c|}
\hline$N$ & 0.05354300 & -1.70099800 & 0.41154400 \\
\hline & -0.25604600 & -1.82167200 & 1.72567600 \\
\hline & -0.42141700 & 1.08168500 & 1.26247900 \\
\hline & -0.96760800 & 0.55817500 & 2.38477600 \\
\hline & -2.62298200 & -0.72813200 & 0.17486700 \\
\hline $\mathrm{N}$ & -2.08430800 & 1.41879400 & -1.69275200 \\
\hline $\mathrm{N}$ & 0.08707900 & -0.82631300 & -2.55222200 \\
\hline C & 0.96204800 & -2.64210100 & 0.12272900 \\
\hline $\mathrm{C}$ & 1.25309500 & -3.39405400 & 1.26067100 \\
\hline $\mathrm{C}$ & 0.45967200 & -2.83645300 & 2.25299400 \\
\hline & -0.25278600 & 2.39627100 & 1.47756400 \\
\hline S & -0.72605400 & 2.74282300 & 2.74162200 \\
\hline & -1.18168700 & 1.54420500 & 3.27611500 \\
\hline & .48298700 & -1.52425700 & 3.94989900 \\
\hline
\end{tabular}




\begin{tabular}{|c|c|c|c|}
\hline $\mathrm{C}$ & -2.18276600 & -2.70580000 & 4.26273600 \\
\hline $\mathrm{C}$ & -2.16700500 & -3.27079500 & 5.53601500 \\
\hline C & -1.43281200 & -2.69252900 & 6.57651900 \\
\hline $\mathrm{C}$ & -0.68765200 & -1.55019600 & 6.27365600 \\
\hline $\mathrm{C}$ & -0.70735100 & -0.99226500 & 4.99568800 \\
\hline $\mathrm{C}$ & -1.44710800 & -3.27627400 & 7.96391800 \\
\hline $\mathrm{C}$ & -2.74553800 & -1.06641600 & 1.49647200 \\
\hline C & -3.97074500 & -1.50219900 & 1.97984100 \\
\hline $\mathrm{C}$ & -5.02944800 & -1.72725700 & 1.08855200 \\
\hline C & -4.85657800 & -1.48439500 & -0.25257200 \\
\hline C & -3.64531400 & -0.90525300 & -0.71800000 \\
\hline $\mathrm{C}$ & -3.55000500 & -0.48686700 & -2.09857800 \\
\hline C & -4.44432200 & -1.20343700 & -3.08881400 \\
\hline $\mathrm{C}$ & -1.77751600 & 2.71215200 & -1.95394900 \\
\hline $\mathrm{C}$ & -2.23652100 & 3.40338400 & -3.04697500 \\
\hline $\mathrm{C}$ & -3.02659600 & 2.68801300 & -3.97458800 \\
\hline C & -3.38293500 & 1.39011100 & -3.71968700 \\
\hline $\mathrm{C}$ & -2.98918500 & 0.74766500 & -2.49644500 \\
\hline $\mathrm{C}$ & -0.24846100 & -1.91709600 & -3.26213100 \\
\hline C & 0.63412900 & -2.55143900 & -4.11640300 \\
\hline C & 1.94063300 & -2.03495700 & -4.20957800 \\
\hline $\mathrm{C}$ & 2.29637300 & -0.90603900 & -3.49986300 \\
\hline $\mathrm{C}$ & 1.33465600 & -0.26295000 & -2.68165400 \\
\hline B & -1.39795100 & -0.96417100 & 2.42705400 \\
\hline $\mathrm{H}$ & 1.36150500 & -2.71877800 & -0.87909000 \\
\hline $\mathrm{H}$ & 1.93627400 & -4.22463500 & 1.35428500 \\
\hline $\mathrm{H}$ & 0.35280100 & -3.10195500 & 3.29263700 \\
\hline $\mathrm{H}$ & 0.21057300 & 3.01027100 & 0.71761300 \\
\hline $\mathrm{H}$ & -0.73038000 & 3.71577700 & 3.20923000 \\
\hline $\mathrm{H}$ & -1.63857900 & 1.33471500 & 4.23095500 \\
\hline $\mathrm{H}$ & -2.73376700 & -3.22413200 & 3.48324200 \\
\hline $\mathrm{H}$ & -2.72820200 & -4.18485600 & 5.72126400 \\
\hline $\mathrm{H}$ & -0.07041800 & -1.09406900 & 7.04545100 \\
\hline $\mathrm{H}$ & -0.06999900 & -0.13193600 & 4.81160600 \\
\hline $\mathrm{H}$ & -2.28205100 & -2.87532400 & 8.55214300 \\
\hline $\mathrm{H}$ & -1.56170800 & -4.36444700 & 7.94067500 \\
\hline $\mathrm{H}$ & -0.52627300 & -3.04326200 & 8.50706600 \\
\hline $\mathrm{H}$ & -4.09780800 & -1.69280600 & 3.03742100 \\
\hline $\mathrm{H}$ & -5.98597300 & -2.09147200 & 1.45633200 \\
\hline $\mathrm{H}$ & -5.67234800 & -1.64269200 & -0.94731100 \\
\hline $\mathrm{H}$ & -5.43430700 & -0.73899800 & -3.22180900 \\
\hline $\mathrm{H}$ & -4.61089500 & -2.24095900 & -2.78542600 \\
\hline $\mathrm{H}$ & -3.97916200 & -1.24502600 & -4.07963100 \\
\hline $\mathrm{H}$ & -1.09657100 & 3.17866500 & -1.24910100 \\
\hline $\mathrm{H}$ & -1.95242900 & 4.43660200 & -3.20806200 \\
\hline $\mathrm{H}$ & -3.36846800 & 3.17095500 & -4.88670200 \\
\hline
\end{tabular}




\begin{tabular}{|c|c|c|c|}
\hline $\mathrm{H}$ & -4.02371000 & 0.86223300 & -4.41443700 \\
\hline $\mathrm{H}$ & -1.26259300 & -2.28025400 & -3.11177800 \\
\hline $\mathrm{H}$ & 0.32599500 & -3.42701700 & -4.67637000 \\
\hline $\mathrm{H}$ & 2.67134200 & -2.52302200 & -4.85033100 \\
\hline $\mathrm{H}$ & 3.29495600 & -0.48699500 & -3.56661800 \\
\hline Sc & -0.75427100 & 0.05282600 & -0.68466400 \\
\hline $\mathrm{P}$ & 1.46578000 & 1.26148900 & -1.76168100 \\
\hline $\mathrm{C}$ & 3.27617100 & 1.64075600 & -1.63790100 \\
\hline $\mathrm{C}$ & 4.11685300 & 1.04786500 & -0.66028800 \\
\hline $\mathrm{C}$ & 3.81085800 & 2.62674100 & -2.51182800 \\
\hline $\mathrm{C}$ & 5.45577500 & 1.44634700 & -0.57950600 \\
\hline $\mathrm{C}$ & 5.15779400 & 2.98720700 & -2.39259200 \\
\hline $\mathrm{C}$ & 5.97840900 & 2.40679400 & -1.43524100 \\
\hline $\mathrm{H}$ & 6.09948000 & 0.99409500 & 0.17098700 \\
\hline $\mathrm{H}$ & 5.56805700 & 3.73847600 & -3.06296900 \\
\hline $\mathrm{H}$ & 7.02160000 & 2.70232300 & -1.35541300 \\
\hline $\mathrm{C}$ & 3.63453700 & -0.00993200 & 0.31807400 \\
\hline $\mathrm{H}$ & 2.58569100 & -0.21645300 & 0.08229800 \\
\hline $\mathrm{C}$ & 2.98740000 & 3.32346700 & -3.58751500 \\
\hline $\mathrm{H}$ & 1.97640000 & 2.90210400 & -3.54086000 \\
\hline $\mathrm{C}$ & 2.86996200 & 4.82944800 & -3.31849300 \\
\hline $\mathrm{H}$ & 2.43550600 & 5.02149000 & -2.33209100 \\
\hline $\mathrm{H}$ & 3.84648400 & 5.32603700 & -3.35301100 \\
\hline $\mathrm{H}$ & 2.22971200 & 5.30487500 & -4.07010400 \\
\hline C & 3.53637400 & 3.05197500 & -4.99441100 \\
\hline $\mathrm{H}$ & 3.58155500 & 1.97815000 & -5.20133000 \\
\hline $\mathrm{H}$ & 2.89219100 & 3.51367100 & -5.75104900 \\
\hline $\mathrm{H}$ & 4.54508900 & 3.46014200 & -5.12592700 \\
\hline $\mathrm{C}$ & 4.41709300 & -1.32137200 & 0.16568000 \\
\hline $\mathrm{H}$ & 4.36554900 & -1.69691400 & -0.86143100 \\
\hline $\mathrm{H}$ & 5.47557200 & -1.19539400 & 0.42066900 \\
\hline $\mathrm{H}$ & 4.00416600 & -2.08776700 & 0.82956600 \\
\hline $\mathrm{C}$ & 3.68361700 & 0.49553700 & 1.76621700 \\
\hline $\mathrm{H}$ & 3.08840900 & 1.40568300 & 1.88637200 \\
\hline $\mathrm{H}$ & 3.28355800 & -0.26295000 & 2.44772500 \\
\hline $\mathrm{H}$ & 4.70925600 & 0.72075900 & 2.08099400 \\
\hline & & & \\
\hline & 0.51303900 & -1.24839600 & 1.17617700 \\
\hline $\mathrm{N}$ & 0.02877700 & -0.88035300 & 2.38535000 \\
\hline $\mathrm{N}$ & 0.58130800 & 1.66711900 & 0.82090300 \\
\hline
\end{tabular}




\begin{tabular}{|c|c|c|c|}
\hline $\mathrm{N}$ & 0.13213000 & 1.67633900 & 2.09839700 \\
\hline $\mathrm{N}$ & -2.12156900 & 0.17881700 & 0.44906800 \\
\hline $\mathrm{N}$ & -1.15184300 & 1.13309500 & -2.24499600 \\
\hline $\mathrm{N}$ & -1.20222700 & -1.66396000 & -1.63845800 \\
\hline $\mathrm{C}$ & 1.18734000 & -2.38945600 & 1.34790600 \\
\hline $\mathrm{C}$ & 1.13122500 & -2.79342100 & 2.68372700 \\
\hline $\mathrm{C}$ & 0.39102400 & -1.80260200 & 3.30665900 \\
\hline $\mathrm{C}$ & 1.13936600 & 2.86179000 & 0.59281100 \\
\hline $\mathrm{C}$ & 1.02561800 & 3.67994400 & 1.71969400 \\
\hline $\mathrm{C}$ & 0.37051200 & 2.88611200 & 2.64873500 \\
\hline $\mathrm{C}$ & -0.96285900 & 0.55655100 & 4.24571200 \\
\hline $\mathrm{C}$ & -2.10730300 & 0.02983900 & 4.86904800 \\
\hline $\mathrm{C}$ & -2.24708400 & -0.02022700 & 6.25551200 \\
\hline $\mathrm{C}$ & -1.23842900 & 0.45059300 & 7.10097500 \\
\hline $\mathrm{C}$ & -0.07447700 & 0.94176400 & 6.50053500 \\
\hline $\mathrm{C}$ & 0.05913400 & 0.98292600 & 5.11421200 \\
\hline $\mathrm{C}$ & -1.39999700 & 0.43670800 & 8.59773800 \\
\hline $\mathrm{C}$ & -2.10922900 & 0.60330200 & 1.73873500 \\
\hline C & -3.21755100 & 1.29616600 & 2.24953600 \\
\hline $\mathrm{C}$ & -4.34042100 & 1.49488300 & 1.46454200 \\
\hline $\mathrm{C}$ & -4.35644200 & 0.98690300 & 0.17133700 \\
\hline $\mathrm{C}$ & -3.21947200 & 0.33599400 & -0.31109000 \\
\hline $\mathrm{C}$ & -3.18939000 & -0.19319800 & -1.77047200 \\
\hline C & -4.62690000 & -0.30484300 & -2.30671900 \\
\hline $\mathrm{C}$ & -0.43522200 & 2.02200600 & -2.94832400 \\
\hline $\mathrm{C}$ & -0.92657600 & 2.66820600 & -4.07430000 \\
\hline $\mathrm{C}$ & -2.20424900 & 2.33302500 & -4.50655000 \\
\hline C & -2.94095600 & 1.39616700 & -3.78940700 \\
\hline $\mathrm{C}$ & -2.39623300 & 0.81521000 & -2.63778900 \\
\hline $\mathrm{C}$ & -0.57150400 & -2.84039000 & -1.75496000 \\
\hline $\mathrm{C}$ & -1.23558100 & -4.03079800 & -2.01845400 \\
\hline $\mathrm{C}$ & -2.61458300 & -3.97862700 & -2.17782900 \\
\hline $\mathrm{C}$ & -3.26392200 & -2.74997500 & -2.09638000 \\
\hline $\mathrm{C}$ & -2.52786300 & -1.58960700 & -1.83088500 \\
\hline B & -0.74171700 & 0.47183700 & 2.63772900 \\
\hline $\mathrm{H}$ & 1.69757200 & -2.84259300 & 0.50930300 \\
\hline $\mathrm{H}$ & 1.57680100 & -3.66548800 & 3.13814200 \\
\hline $\mathrm{H}$ & 0.11127700 & -1.68898700 & 4.34156200 \\
\hline $\mathrm{H}$ & 1.61456700 & 3.05944800 & -0.35803700 \\
\hline $\mathrm{H}$ & 1.37965400 & 4.69147500 & 1.85040200 \\
\hline $\mathrm{H}$ & 0.06207100 & 3.10388600 & 3.65939500 \\
\hline $\mathrm{H}$ & -2.91104800 & -0.37646000 & 4.25936200 \\
\hline $\mathrm{H}$ & -3.15372700 & -0.44001300 & 6.68751500 \\
\hline $\mathrm{H}$ & 0.74634100 & 1.28527900 & 7.12740300 \\
\hline $\mathrm{H}$ & 1.00273700 & 1.33112900 & 4.70140100 \\
\hline $\mathrm{H}$ & -0.44068900 & 0.28956900 & 9.10344700 \\
\hline
\end{tabular}




\begin{tabular}{|c|c|c|c|}
\hline $\mathrm{H}$ & -1.81448000 & 1.38604100 & 8.95967900 \\
\hline $\mathrm{H}$ & -2.07923600 & -0.35800400 & 8.92084600 \\
\hline $\mathrm{H}$ & -3.18100800 & 1.68228600 & 3.26151900 \\
\hline $\mathrm{H}$ & -5.20245700 & 2.03325900 & 1.85034600 \\
\hline $\mathrm{H}$ & -5.23821800 & 1.11864300 & -0.44083400 \\
\hline $\mathrm{H}$ & -5.09239000 & 0.67662300 & -2.40618800 \\
\hline $\mathrm{H}$ & -5.24857200 & -0.89669700 & -1.63137700 \\
\hline $\mathrm{H}$ & -4.64045300 & -0.77856300 & -3.29135500 \\
\hline $\mathrm{H}$ & 0.58269600 & 2.17592900 & -2.60205600 \\
\hline $\mathrm{H}$ & -0.31071700 & 3.38588700 & -4.60490000 \\
\hline $\mathrm{H}$ & -2.62643800 & 2.78655300 & -5.39886200 \\
\hline $\mathrm{H}$ & -3.92952700 & 1.12759000 & -4.13655700 \\
\hline $\mathrm{H}$ & 0.50857200 & -2.79334800 & -1.64733700 \\
\hline $\mathrm{H}$ & -0.68259500 & -4.95996900 & -2.10170500 \\
\hline $\mathrm{H}$ & -3.18582300 & -4.88006800 & -2.38092700 \\
\hline $\mathrm{H}$ & -4.33515100 & -2.71225700 & -2.24072100 \\
\hline Sc & 0.12308100 & -0.02328400 & -0.66175000 \\
\hline $\mathrm{C}$ & 2.79649300 & -0.34193100 & -2.41128800 \\
\hline C & 4.07707500 & -0.03289500 & -1.83361700 \\
\hline $\mathrm{C}$ & 2.79552500 & -0.80496700 & -3.77203000 \\
\hline $\mathrm{C}$ & 5.24295700 & -0.18761400 & -2.58032200 \\
\hline $\mathrm{C}$ & 3.99305300 & -0.94036100 & -4.47387700 \\
\hline $\mathrm{C}$ & 5.22355200 & -0.63843200 & -3.89832400 \\
\hline $\mathrm{H}$ & 6.19939400 & 0.05115400 & -2.11625300 \\
\hline $\mathrm{H}$ & 3.96271300 & -1.29452100 & -5.50409400 \\
\hline $\mathrm{H}$ & 6.14566300 & -0.75189700 & -4.46236500 \\
\hline $\mathrm{C}$ & 4.17152000 & 0.46039500 & -0.40102800 \\
\hline $\mathrm{H}$ & 3.14274600 & 0.52826400 & -0.03230100 \\
\hline $\mathrm{C}$ & 1.49123900 & -1.15876900 & -4.46399400 \\
\hline $\mathrm{H}$ & 0.69856400 & -0.96415900 & -3.73225000 \\
\hline $\mathrm{C}$ & 1.22888400 & -0.27784300 & -5.69360600 \\
\hline $\mathrm{H}$ & 1.24411900 & 0.78367700 & -5.42756500 \\
\hline $\mathrm{H}$ & 1.99140300 & -0.43220000 & -6.46616600 \\
\hline $\mathrm{H}$ & 0.25341700 & -0.50397100 & -6.14360500 \\
\hline $\mathrm{C}$ & 1.43140100 & -2.64275300 & -4.85442400 \\
\hline $\mathrm{H}$ & 1.60953900 & -3.28855200 & -3.98824500 \\
\hline $\mathrm{H}$ & 0.45467100 & -2.90375800 & -5.28231800 \\
\hline $\mathrm{H}$ & 2.19497700 & -2.88877900 & -5.60178500 \\
\hline $\mathrm{C}$ & 4.92476400 & -0.52897100 & 0.49874300 \\
\hline $\mathrm{H}$ & 4.45282200 & -1.51633000 & 0.47663600 \\
\hline $\mathrm{H}$ & 5.96587800 & -0.65270800 & 0.17659800 \\
\hline $\mathrm{H}$ & 4.93756800 & -0.18319000 & 1.53990600 \\
\hline $\mathrm{C}$ & 4.80125900 & 1.85726300 & -0.31205400 \\
\hline $\mathrm{H}$ & 4.26477500 & 2.57407800 & -0.94323800 \\
\hline $\mathrm{H}$ & 4.78421200 & 2.23148500 & 0.71901700 \\
\hline $\mathrm{H}$ & 5.84642200 & 1.85113400 & -0.64428900 \\
\hline
\end{tabular}




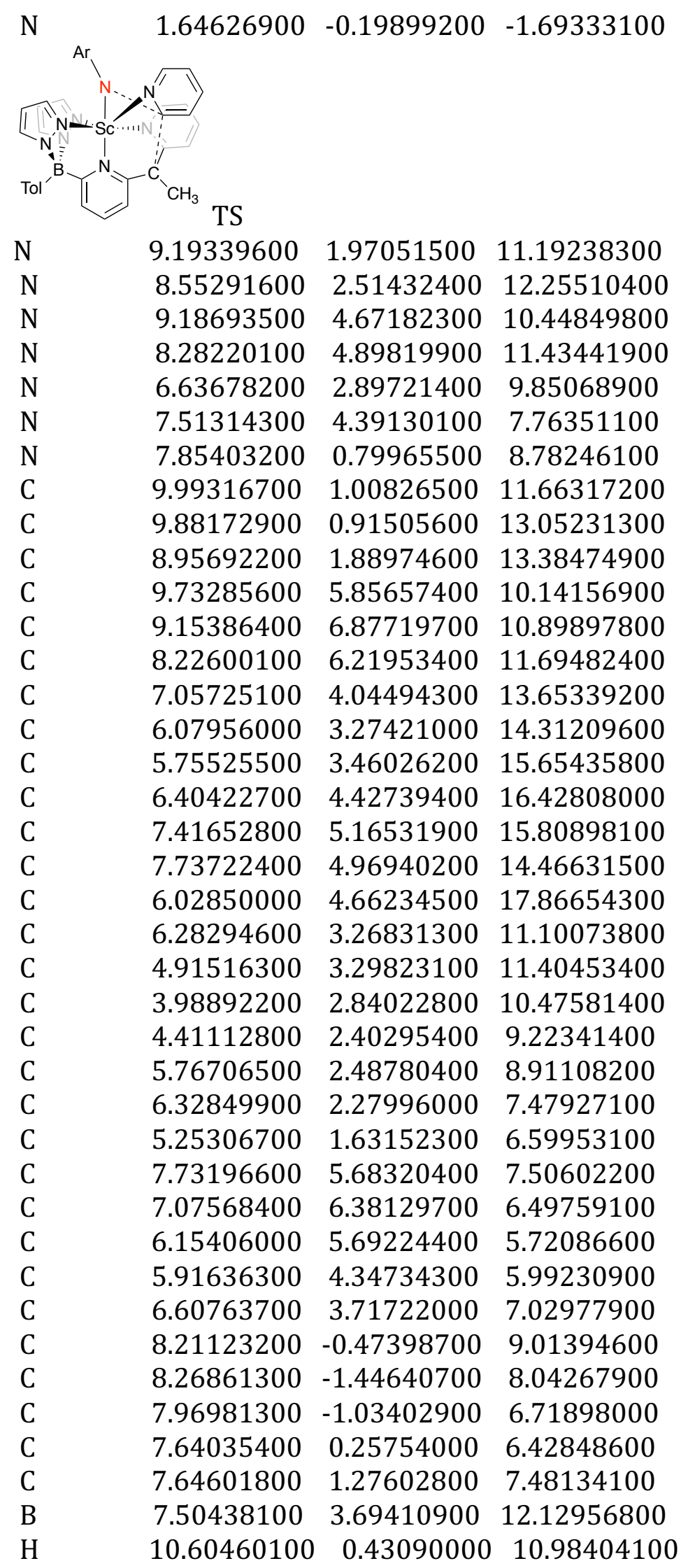




\begin{tabular}{|c|c|c|c|}
\hline $\mathrm{H}$ & 10.39557700 & 0.23960400 & 13.71946200 \\
\hline $\mathrm{H}$ & 8.56040200 & 2.17653900 & 14.34526300 \\
\hline $\mathrm{H}$ & 10.51160100 & 5.91332500 & 9.39147100 \\
\hline $\mathrm{H}$ & 9.37808200 & 7.93326700 & 10.88403800 \\
\hline $\mathrm{H}$ & 7.54332500 & 6.60736500 & 12.43502000 \\
\hline $\mathrm{H}$ & 5.57796100 & 2.47228400 & 13.77737900 \\
\hline $\mathrm{H}$ & 4.99138800 & 2.83369900 & 16.11101100 \\
\hline $\mathrm{H}$ & 7.97579500 & 5.89686500 & 16.38925000 \\
\hline $\mathrm{H}$ & 8.56865000 & 5.53786600 & 14.05977200 \\
\hline $\mathrm{H}$ & 6.87743600 & 5.03569500 & 18.44722200 \\
\hline $\mathrm{H}$ & 5.22631200 & 5.40644800 & 17.94852200 \\
\hline $\mathrm{H}$ & 5.66858400 & 3.74503500 & 18.34249400 \\
\hline $\mathrm{H}$ & 4.58220100 & 3.67293300 & 12.36438800 \\
\hline $\mathrm{H}$ & 2.92970300 & 2.82960900 & 10.72058800 \\
\hline $\mathrm{H}$ & 3.68768300 & 2.03487200 & 8.50795400 \\
\hline $\mathrm{H}$ & 4.32605900 & 2.21109700 & 6.59916200 \\
\hline $\mathrm{H}$ & 5.03726300 & 0.62132600 & 6.95330700 \\
\hline $\mathrm{H}$ & 5.58902700 & 1.55168800 & 5.56499100 \\
\hline $\mathrm{H}$ & 8.45403300 & 6.18025200 & 8.14474000 \\
\hline $\mathrm{H}$ & 7.28953300 & 7.43245100 & 6.33538200 \\
\hline $\mathrm{H}$ & 5.61884900 & 6.18976100 & 4.91699400 \\
\hline $\mathrm{H}$ & 5.19665300 & 3.79824500 & 5.39883600 \\
\hline $\mathrm{H}$ & 8.42308400 & -0.70384600 & 10.05744000 \\
\hline $\mathrm{H}$ & 8.50408600 & -2.47536900 & 8.28720900 \\
\hline $\mathrm{H}$ & 7.99227800 & -1.76090700 & 5.90948300 \\
\hline $\mathrm{H}$ & 7.43891300 & 0.54615300 & 5.40573500 \\
\hline Sc & 8.71724200 & 2.80443500 & 9.13791300 \\
\hline $\mathrm{C}$ & 10.11288900 & 1.84689600 & 6.39374700 \\
\hline $\mathrm{C}$ & 11.35820400 & 1.31290800 & 6.86630000 \\
\hline $\mathrm{C}$ & 9.92606100 & 1.92693800 & 4.98010300 \\
\hline $\mathrm{C}$ & 12.31208400 & 0.84922800 & 5.96442000 \\
\hline $\mathrm{C}$ & 10.90469800 & 1.42666800 & 4.11690900 \\
\hline $\mathrm{C}$ & 12.08888500 & 0.87818500 & 4.58981400 \\
\hline $\mathrm{H}$ & 13.24838600 & 0.44306700 & 6.33714300 \\
\hline $\mathrm{H}$ & 10.74513200 & 1.49137600 & 3.04302000 \\
\hline $\mathrm{H}$ & 12.83658400 & 0.49473700 & 3.90080300 \\
\hline $\mathrm{C}$ & 11.64195600 & 1.30929300 & 8.35545900 \\
\hline $\mathrm{H}$ & 10.68962800 & 1.03628200 & 8.83565300 \\
\hline $\mathrm{C}$ & 8.75872300 & 2.69829000 & 4.39080500 \\
\hline $\mathrm{H}$ & 7.98500900 & 2.75449000 & 5.16099000 \\
\hline $\mathrm{C}$ & 9.19659600 & 4.14054000 & 4.08899800 \\
\hline $\mathrm{H}$ & 9.58533800 & 4.62372400 & 4.98998600 \\
\hline $\mathrm{H}$ & 9.99073200 & 4.14982400 & 3.33357300 \\
\hline $\mathrm{H}$ & 8.36070900 & 4.74040300 & 3.70981500 \\
\hline $\mathrm{C}$ & 8.14202100 & 2.06252900 & 3.13933000 \\
\hline $\mathrm{H}$ & 7.88417600 & 1.01012000 & 3.29724800 \\
\hline
\end{tabular}




$\begin{array}{cccc}\mathrm{H} & 7.23159500 & 2.59864900 & 2.84614700 \\ \mathrm{H} & 8.82346100 & 2.10526100 & 2.28249400 \\ \mathrm{C} & 12.68224600 & 0.28407000 & 8.81066300 \\ \mathrm{H} & 12.42649400 & -0.72304200 & 8.46811700 \\ \mathrm{H} & 13.68338500 & 0.52643800 & 8.43676900 \\ \mathrm{H} & 12.75143300 & 0.26850400 & 9.90455800 \\ \mathrm{C} & 12.04334600 & 2.71583200 & 8.83352300 \\ \mathrm{H} & 11.32951300 & 3.47375300 & 8.48244100 \\ \mathrm{H} & 12.11511000 & 2.77466500 & 9.92579300 \\ \mathrm{H} & 13.01568800 & 2.99442900 & 8.41161000 \\ \mathrm{~N} & 9.17806600 & 2.29202000 & 7.30540800\end{array}$

$\begin{array}{lrrr} & & \\ \mathrm{N}\end{array}$




$\begin{array}{lrrr}\mathrm{C} & -1.85929300 & 1.93637700 & -4.37427500 \\ \mathrm{C} & -3.05058900 & 1.24139400 & -4.69293100 \\ \mathrm{C} & -3.67531500 & 0.47606300 & -3.74603400 \\ \mathrm{C} & -3.15915500 & 0.40458500 & -2.40467600 \\ \mathrm{C} & -1.45222000 & -3.24743900 & -2.11141700 \\ \mathrm{C} & -0.87861900 & -4.32311200 & -2.76242400 \\ \mathrm{C} & 0.47422100 & -4.21038800 & -3.13579000 \\ \mathrm{C} & 1.18861700 & -3.05920700 & -2.87826900 \\ \mathrm{C} & 0.53234000 & -1.97530400 & -2.23006400 \\ \mathrm{~B} & -0.78254400 & 0.80407800 & 2.46946800 \\ \mathrm{H} & -0.01157500 & -3.36046200 & 0.55312500 \\ \mathrm{H} & 0.70648000 & -3.68458200 & 3.19350000 \\ \mathrm{H} & 0.32605300 & -1.18456100 & 4.25671200 \\ \mathrm{H} & 1.47635700 & 2.25982800 & -1.28632300 \\ \mathrm{H} & 1.63338900 & 4.40763800 & 0.43191500 \\ \mathrm{H} & 0.32980100 & 3.50995500 & 2.67572800 \\ \mathrm{H} & -2.40410100 & 0.21124500 & 4.71020800 \\ \mathrm{H} & -2.03038900 & 0.55436200 & 7.09257200 \\ \mathrm{H} & 1.78617600 & 2.31077100 & 6.24444000 \\ \mathrm{H} & 1.41924800 & 1.96811900 & 3.86447600 \\ \mathrm{H} & 1.17557200 & 1.74287600 & 8.58030900 \\ \mathrm{H} & -0.30997300 & 2.69236900 & 8.64739000 \\ \mathrm{H} & -0.37020000 & 0.95338400 & 8.93491100 \\ \mathrm{H} & -3.24560400 & 1.76647000 & 3.57347000 \\ \mathrm{H} & -5.54843700 & 1.52117500 & 2.64430900 \\ \mathrm{H} & -5.85233500 & 0.52747900 & 0.40591400 \\ \mathrm{H} & -6.04441400 & -0.04439200 & -1.96946100 \\ \mathrm{H} & -5.63391100 & -1.32284200 & -0.81924600 \\ \mathrm{H} & -5.14857600 & -1.46925700 & -2.49541600 \\ \mathrm{H} & -0.35904300 & 2.14025900 & -2.85807300 \\ \mathrm{H} & -1.36064900 & 2.58101400 & -5.08760000 \\ \mathrm{H} & -3.48317600 & 1.32930400 & -5.68651900 \\ \mathrm{H} & -4.61002900 & -0.01732500 & -3.98161100 \\ \mathrm{H} & -2.48700200 & -3.26500700 & -1.77801400 \\ \mathrm{H} & -1.45420300 & -5.21813100 & -2.96731000 \\ \mathrm{H} & 0.96703900 & -5.04302200 & -3.63239400 \\ \mathrm{H} & 2.23329700 & -2.96197800 & -3.15039800 \\ \mathrm{H} & -0.78833500 & -0.20715100 & -0.73071500 \\ \mathrm{H} & & & \end{array}$




$\begin{array}{lccc}\mathrm{H} & 6.07685300 & 0.39645700 & -3.12978400 \\ \mathrm{C} & 3.12480000 & -1.12127800 & 0.14987100 \\ \mathrm{H} & 2.04106100 & -1.12428700 & 0.28359100 \\ \mathrm{C} & 1.65888400 & 0.19389000 & -4.60744100 \\ \mathrm{H} & 0.67452500 & 0.05067600 & -4.15174500 \\ \mathrm{C} & 1.69687300 & 1.61261200 & -5.19316600 \\ \mathrm{H} & 1.60093300 & 2.37606500 & -4.41415400 \\ \mathrm{H} & 2.63356700 & 1.80642400 & -5.72700100 \\ \mathrm{H} & 0.87897200 & 1.74985400 & -5.90800000 \\ \mathrm{C} & 1.80977700 & -0.84093800 & -5.73318200 \\ \mathrm{H} & 1.72870900 & -1.86253400 & -5.35265300 \\ \mathrm{H} & 1.02920100 & -0.70134600 & -6.48952600 \\ \mathrm{H} & 2.78067200 & -0.74278400 & -6.23260100 \\ \mathrm{C} & 3.61913500 & -2.56842600 & 0.29923900 \\ \mathrm{H} & 3.16359300 & -3.22497200 & -0.44894200 \\ \mathrm{H} & 4.70700700 & -2.62829700 & 0.17613900 \\ \mathrm{H} & 3.36889700 & -2.95970400 & 1.29130300 \\ \mathrm{C} & 3.71662800 & -0.23044400 & 1.24935000 \\ \mathrm{H} & 3.38182700 & 0.80552800 & 1.14327500 \\ \mathrm{H} & 3.40014800 & -0.59108300 & 2.23374200 \\ \mathrm{H} & 4.81261400 & -0.23367800 & 1.23748900 \\ \mathrm{~N} & 1.03467000 & -0.77447000 & -1.88395700\end{array}$

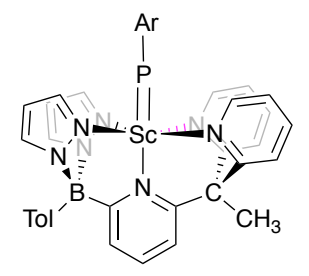

$\begin{array}{cccc}\mathrm{N} & 9.21954800 & 2.49332400 & 11.17588900 \\ \mathrm{~N} & 8.47075400 & 2.43992200 & 12.30243900 \\ \mathrm{~N} & 8.26793200 & 5.23285700 & 11.18886700 \\ \mathrm{~N} & 7.65341600 & 4.87209600 & 12.34017800 \\ \mathrm{~N} & 6.40449800 & 3.04112800 & 10.09074500 \\ \mathrm{~N} & 7.26962600 & 4.74588200 & 7.81016000 \\ \mathrm{~N} & 8.21753600 & 1.99229400 & 8.07137000 \\ \mathrm{C} & 10.27089600 & 1.68691400 & 11.35586500 \\ \mathrm{C} & 10.20243900 & 1.07210100 & 12.60752300 \\ \mathrm{C} & 9.04827400 & 1.58487800 & 13.17663600 \\ \mathrm{C} & 8.41259200 & 6.56332400 & 11.21974100 \\ \mathrm{C} & 7.84954100 & 7.08843600 & 12.38559500 \\ \mathrm{C} & 7.37294600 & 5.97643000 & 13.06378700 \\ \mathrm{C} & 6.74128800 & 3.06435600 & 14.06983300 \\ \mathrm{C} & 5.81525400 & 2.04987500 & 14.37033000 \\ \mathrm{C} & 5.49704700 & 1.69217800 & 15.67958700 \\ \mathrm{C} & 6.09863100 & 2.32899000 & 16.76920200\end{array}$




\begin{tabular}{|c|c|c|c|}
\hline $\mathrm{C}$ & 7.05308400 & 3.31244100 & 16.49117100 \\
\hline $\mathrm{C}$ & 7.36937300 & 3.65991500 & 15.17911200 \\
\hline $\mathrm{C}$ & 5.72920400 & 1.97484700 & 18.18506100 \\
\hline $\mathrm{C}$ & 6.06235800 & 3.17721100 & 11.39965400 \\
\hline $\mathrm{C}$ & 4.71106000 & 3.30721300 & 11.75186100 \\
\hline $\mathrm{C}$ & 3.72466600 & 3.23246700 & 10.78424600 \\
\hline $\mathrm{C}$ & 4.09522200 & 3.00387000 & 9.46539800 \\
\hline $\mathrm{C}$ & 5.45114700 & 2.91727200 & 9.14600800 \\
\hline $\mathrm{C}$ & 5.88283300 & 2.70180300 & 7.67236800 \\
\hline $\mathrm{C}$ & 4.67949700 & 2.20675900 & 6.85106200 \\
\hline $\mathrm{C}$ & 7.65787000 & 5.96052200 & 7.38624900 \\
\hline $\mathrm{C}$ & 7.17455200 & 6.54847400 & 6.22659500 \\
\hline $\mathrm{C}$ & 6.28374600 & 5.81547100 & 5.44874900 \\
\hline C & 5.87552700 & 4.56228400 & 5.88708600 \\
\hline $\mathrm{C}$ & 6.36431200 & 4.05493300 & 7.09920100 \\
\hline $\mathrm{C}$ & 9.24211500 & 1.12984500 & 7.96579000 \\
\hline C & 9.11245800 & -0.13883800 & 7.42126900 \\
\hline C & 7.86273400 & -0.51621400 & 6.94226600 \\
\hline $\mathrm{C}$ & 6.81001300 & 0.38934700 & 7.01520800 \\
\hline $\mathrm{C}$ & 7.01099200 & 1.65095200 & 7.58825200 \\
\hline B & 7.21990900 & 3.36618400 & 12.54668500 \\
\hline $\mathrm{H}$ & 11.03091500 & 1.62792000 & 10.58938000 \\
\hline $\mathrm{H}$ & 10.89785400 & 0.37329900 & 13.04752900 \\
\hline $\mathrm{H}$ & 8.61151400 & 1.40850800 & 14.14642300 \\
\hline $\mathrm{H}$ & 8.94044500 & 7.05785400 & 10.41607900 \\
\hline $\mathrm{H}$ & 7.80909600 & 8.11977900 & 12.70246800 \\
\hline $\mathrm{H}$ & 6.85829700 & 5.90229400 & 14.00910100 \\
\hline $\mathrm{H}$ & 5.33869500 & 1.50202200 & 13.56065700 \\
\hline $\mathrm{H}$ & 4.77415600 & 0.89801100 & 15.85727800 \\
\hline $\mathrm{H}$ & 7.56791200 & 3.80521800 & 17.31382000 \\
\hline $\mathrm{H}$ & 8.15409200 & 4.39526500 & 15.02049100 \\
\hline $\mathrm{H}$ & 4.89130100 & 2.58780100 & 18.54037900 \\
\hline $\mathrm{H}$ & 5.42316000 & 0.92762700 & 18.27016500 \\
\hline $\mathrm{H}$ & 6.56532200 & 2.14095500 & 18.87114600 \\
\hline $\mathrm{H}$ & 4.45042700 & 3.47825000 & 12.78952000 \\
\hline $\mathrm{H}$ & 2.67523200 & 3.33967600 & 11.04662800 \\
\hline $\mathrm{H}$ & 3.32978000 & 2.91562000 & 8.70710600 \\
\hline $\mathrm{H}$ & 3.91296000 & 2.97866100 & 6.76684600 \\
\hline $\mathrm{H}$ & 4.22299600 & 1.32999300 & 7.31589500 \\
\hline $\mathrm{H}$ & 4.98459600 & 1.93494800 & 5.83811100 \\
\hline $\mathrm{H}$ & 8.42088700 & 6.43943300 & 7.99152700 \\
\hline $\mathrm{H}$ & 7.51781800 & 7.53407800 & 5.93231100 \\
\hline $\mathrm{H}$ & 5.90637100 & 6.21214100 & 4.51033000 \\
\hline $\mathrm{H}$ & 5.18020800 & 3.99347900 & 5.28463000 \\
\hline $\mathrm{H}$ & 10.19588400 & 1.51585800 & 8.31667300 \\
\hline $\mathrm{H}$ & 9.97177800 & -0.79815300 & 7.36624700 \\
\hline
\end{tabular}




$\begin{array}{lccc}\mathrm{H} & 7.70609300 & -1.49665600 & 6.50145600 \\ \mathrm{H} & 5.84199200 & 0.10055200 & 6.62903100 \\ \mathrm{Sc} & 8.63393900 & 3.79106500 & 9.46506200 \\ \mathrm{P} & 10.64378800 & 4.50247900 & 8.58251700 \\ \mathrm{C} & 12.06807200 & 5.07438000 & 7.58986200 \\ \mathrm{C} & 12.94923800 & 6.06868600 & 8.10379500 \\ \mathrm{C} & 12.33034600 & 4.54163500 & 6.29549300 \\ \mathrm{C} & 14.03400800 & 6.49717100 & 7.33518300 \\ \mathrm{C} & 13.42894600 & 5.00051200 & 5.56530000 \\ \mathrm{C} & 14.28346700 & 5.97395300 & 6.07111300 \\ \mathrm{H} & 14.70099400 & 7.25719400 & 7.73773600 \\ \mathrm{H} & 13.62097000 & 4.58408600 & 4.57805500 \\ \mathrm{H} & 15.13422200 & 6.31879900 & 5.48869000 \\ \mathrm{C} & 12.74744000 & 6.68349600 & 9.47993500 \\ \mathrm{H} & 11.86125800 & 6.19773900 & 9.90936200 \\ \mathrm{C} & 11.45083300 & 3.46566300 & 5.67873800 \\ \mathrm{H} & 10.63654800 & 3.29068600 & 6.39454700 \\ \mathrm{C} & 10.81652800 & 3.92188400 & 4.35912800 \\ \mathrm{H} & 10.23000500 & 4.83481500 & 4.50331000 \\ \mathrm{H} & 11.57280200 & 4.13067100 & 3.59321300 \\ \mathrm{H} & 10.14963500 & 3.14755600 & 3.96008700 \\ \mathrm{C} & 12.21669300 & 2.14836900 & 5.49870200 \\ \mathrm{H} & 12.63869200 & 1.80764500 & 6.45008800 \\ \mathrm{H} & 11.55705100 & 1.36130700 & 5.11235000 \\ \mathrm{H} & 13.04760600 & 2.25890500 & 4.79189200 \\ \mathrm{C} & 13.92888700 & 6.39588000 & 10.41439900 \\ \mathrm{H} & 14.10300600 & 5.31892800 & 10.50205500 \\ \mathrm{H} & 14.85601200 & 6.85619800 & 10.05236400 \\ \mathrm{H} & 13.73330200 & 6.79085100 & 11.41838500 \\ \mathrm{C} & 12.45876300 & 8.18761500 & 9.39496000 \\ \mathrm{H} & 11.58996100 & 8.38516100 & 8.75784700 \\ \mathrm{H} & 12.25449900 & 8.60086100 & 10.39011800 \\ \mathrm{H} & 13.30745000 & 8.74117600 & 8.97579100 \\ & & & \end{array}$

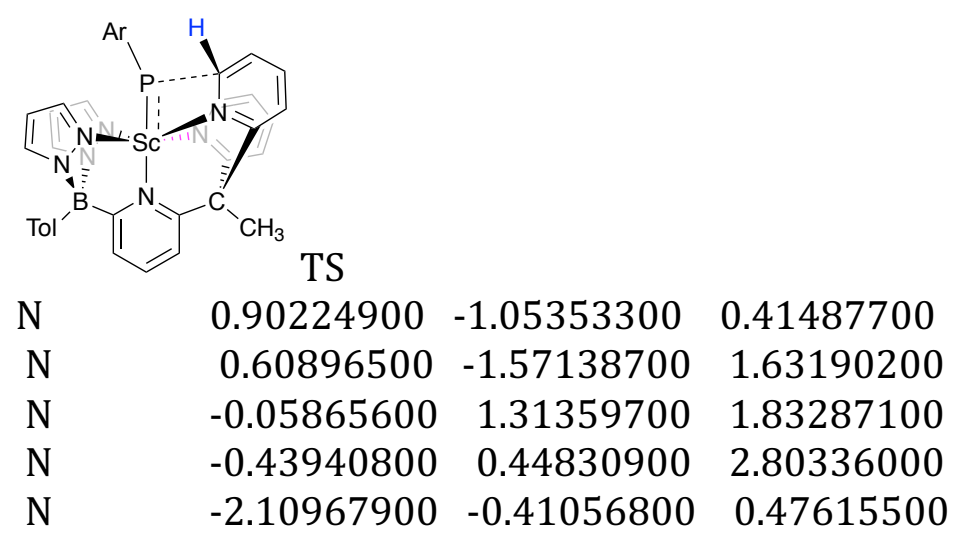




$\begin{array}{lrrr}\mathrm{N} & -1.77250200 & 2.29571700 & -0.75782300 \\ \mathrm{~N} & -1.08175600 & -0.26280500 & -2.11406300 \\ \mathrm{C} & 2.02765100 & -1.64091900 & -0.01510600 \\ \mathrm{C} & 2.47226700 & -2.56827800 & 0.92633900 \\ \mathrm{C} & 1.54673800 & -2.48802100 & 1.95473700 \\ \mathrm{C} & -0.11657800 & 2.54272200 & 2.36701400 \\ \mathrm{C} & -0.57654900 & 2.48906000 & 3.68211600 \\ \mathrm{C} & -0.78339300 & 1.13640600 & 3.91002800 \\ \mathrm{C} & -0.57626100 & -2.00499300 & 3.83787300 \\ \mathrm{C} & -1.04882200 & -3.33201200 & 3.82710400 \\ \mathrm{C} & -0.90952800 & -4.18697200 & 4.91800200 \\ \mathrm{C} & -0.27079200 & -3.76683100 & 6.08934300 \\ \mathrm{C} & 0.25476700 & -2.47244800 & 6.10052100 \\ \mathrm{C} & 0.11337900 & -1.62425700 & 5.00234400 \\ \mathrm{C} & -0.15505500 & -4.67231700 & 7.28606800 \\ \mathrm{C} & -2.02100400 & -1.14024500 & 1.62205300 \\ \mathrm{C} & -3.15136500 & -1.82193300 & 2.08787100 \\ \mathrm{C} & -4.31529900 & -1.83933700 & 1.33695200 \\ \mathrm{C} & -4.35984800 & -1.13199700 & 0.14476700 \\ \mathrm{C} & -3.24624500 & -0.38570100 & -0.25031000 \\ \mathrm{C} & -3.33034800 & 0.53326600 & -1.49449800 \\ \mathrm{C} & -4.73106000 & 0.44385500 & -2.12036200 \\ \mathrm{C} & -1.43047500 & 3.57590700 & -0.54373900 \\ \mathrm{C} & -2.34434200 & 4.61688100 & -0.55601200 \\ \mathrm{C} & -3.67707000 & 4.30686400 & -0.81666800 \\ \mathrm{C} & -4.02529400 & 2.99014500 & -1.09073600 \\ \mathrm{C} & -3.04495300 & 1.99078700 & -1.07984100 \\ \mathrm{C} & -2.27091900 & 0.19457900 & -2.56958300 \\ \mathrm{C} & -2.50723600 & 0.41977900 & -3.90857300 \\ \mathrm{C} & -1.44713400 & 0.18489500 & -4.83043100 \\ \mathrm{C} & -0.21989200 & -0.20116100 & -4.38500800 \\ \mathrm{C} & 0.00615000 & -0.36269800 & -2.97517600 \\ \mathrm{~B} & -0.62137100 & -1.09310300 & 2.49167100 \\ \mathrm{H} & 2.47397100 & -1.33965000 & -0.95314100 \\ \mathrm{H} & 3.34690400 & -3.19866800 & 0.87548000 \\ \mathrm{H} & 1.49732700 & -3.02069600 & 2.89067000 \\ \mathrm{H} & 0.18660500 & 3.40258300 & 1.78737500 \\ \mathrm{H} & -0.72680200 & 3.30541100 & 4.37233000 \\ \mathrm{H} & -1.15080500 & 0.62498000 & 4.78588200 \\ \mathrm{H} & -1.50990200 & -3.73069100 & 2.92672900 \\ \mathrm{H} & -1.29372100 & -5.20322300 & 4.85293400 \\ \mathrm{H} & 0.79756700 & -2.12351600 & 6.97698100 \\ \mathrm{H} & 0.59051800 & -0.64979000 & 5.05372100 \\ \mathrm{H} & -0.06880700 & -5.72197300 & 6.98863000 \\ \mathrm{H} & 0.71659600 & -4.42000700 & 7.89733400 \\ \mathrm{H} & -1.03927700 & -4.58985100 & 7.93048200\end{array}$




$\begin{array}{lccc}\mathrm{H} & -3.11044100 & -2.33499500 & 3.04003200 \\ \mathrm{H} & -5.18742400 & -2.39248400 & 1.67637900 \\ \mathrm{H} & -5.26175400 & -1.15328300 & -0.45013000 \\ \mathrm{H} & -5.51648000 & 0.64889600 & -1.38929100 \\ \mathrm{H} & -4.89580600 & -0.54997100 & -2.54203500 \\ \mathrm{H} & -4.84072400 & 1.17014700 & -2.92607500 \\ \mathrm{H} & -0.36996700 & 3.75782900 & -0.39165900 \\ \mathrm{H} & -2.01642300 & 5.63474500 & -0.37583200 \\ \mathrm{H} & -4.43559700 & 5.08429500 & -0.83171900 \\ \mathrm{H} & -5.05360300 & 2.74906200 & -1.32614000 \\ \mathrm{H} & 0.75831700 & -1.08149900 & -2.66770000 \\ \mathrm{H} & -3.46903600 & 0.74771000 & -4.27632500 \\ \mathrm{H} & -1.62992600 & 0.30967600 & -5.89453400 \\ \mathrm{H} & 0.59701700 & -0.38392400 & -5.07385400 \\ \mathrm{Sc} & -0.18106300 & 0.67215700 & -0.38811000 \\ \mathrm{P} & 1.39432400 & 1.57558600 & -2.15219100 \\ \mathrm{C} & 3.18495700 & 1.20865400 & -1.79060500 \\ \mathrm{C} & 3.84076900 & 1.69426100 & -0.61734600 \\ \mathrm{C} & 3.97270700 & 0.49383500 & -2.73817600 \\ \mathrm{C} & 5.19179800 & 1.41083800 & -0.40436000 \\ \mathrm{C} & 5.32001700 & 0.22286100 & -2.47487300 \\ \mathrm{C} & 5.93475700 & 0.66322600 & -1.31189900 \\ \mathrm{H} & 2.62326700 & 4.70982800 & 0.59501800 \\ \mathrm{H} & 5.67831200 & 1.78286900 & 0.49295300 \\ \mathrm{H} & 5.90038000 & -0.33465300 & -3.20744500 \\ \mathrm{H} & 6.98172500 & 0.44317400 & -1.11961800 \\ \mathrm{C} & 3.12231500 & 2.59079000 & 0.37765200 \\ \mathrm{H} & 2.06546400 & 2.29010000 & 0.35283500 \\ \mathrm{C} & 3.41716800 & 0.04942000 & -4.08306200 \\ \mathrm{H} & 2.36920300 & 0.36574600 & -4.09964100 \\ \mathrm{C} & 4.12219200 & 0.77232800 & -5.23938900 \\ \mathrm{H} & 4.03928200 & 1.85748100 & -5.12685400 \\ \mathrm{H} & 5.18800600 & 0.52015600 & -5.28284800 \\ \mathrm{H} & 3.67605800 & 0.49445000 & -6.20181200 \\ \mathrm{C} & 3.47083300 & -1.47146000 & -4.27760700 \\ \mathrm{H} & 2.94433000 & -2.00217400 & -3.47692900 \\ \mathrm{H} & 3.01218500 & -1.76055000 & -5.23086600 \\ \mathrm{H} & 4.50313800 & -1.83989700 & -4.28864300 \\ \mathrm{H} & 3.60530400 & 2.45855200 & 1.82374500 \\ \mathrm{H} & 3.61854500 & 1.41343700 & 2.14809400 \\ \mathrm{H} & 2.63952900 & 3.01146300 & 2.49535400 \\ \mathrm{H} & 2.18084600 & 4.05435400 & -0.08705200 \\ \mathrm{H} & 4.21790800 & 4.40835500 & -0.11527000\end{array}$




\begin{tabular}{|c|c|c|c|}
\hline $\mathrm{N}$ & 0.85785100 & -1.11819600 & 0.32712300 \\
\hline $\mathrm{N}$ & 0.60198400 & -1.64134500 & 1.55094300 \\
\hline $\mathrm{N}$ & -0.16793200 & 1.22551700 & 1.84334300 \\
\hline $\mathrm{N}$ & -0.53757300 & 0.30831900 & 2.77073900 \\
\hline $\mathrm{N}$ & -2.10848300 & -0.60372200 & 0.34111800 \\
\hline $\mathrm{N}$ & -1.94214600 & 2.15566100 & -0.71757200 \\
\hline $\mathrm{N}$ & -0.96490300 & 0.00764300 & -2.24044700 \\
\hline $\mathrm{C}$ & 1.95860800 & -1.71878600 & -0.14571500 \\
\hline $\mathrm{C}$ & 2.42799900 & -2.65333700 & 0.77652600 \\
\hline $\mathrm{C}$ & 1.54041800 & -2.56955400 & 1.83678800 \\
\hline $\mathrm{C}$ & -0.27674500 & 2.42790300 & 2.42851100 \\
\hline $\mathrm{C}$ & -0.75586100 & 2.30480600 & 3.73158400 \\
\hline $\mathrm{C}$ & -0.92246500 & 0.93763600 & 3.89839000 \\
\hline $\mathrm{C}$ & -0.59119000 & -2.17450000 & 3.72913600 \\
\hline $\mathrm{C}$ & -1.03148200 & -3.51195500 & 3.68883400 \\
\hline $\mathrm{C}$ & -0.86800500 & -4.38816500 & 4.75922700 \\
\hline $\mathrm{C}$ & -0.23538600 & -3.98040500 & 5.93825200 \\
\hline $\mathrm{C}$ & 0.25779300 & -2.67396700 & 5.97873800 \\
\hline $\mathrm{C}$ & 0.09157900 & -1.80411800 & 4.90117900 \\
\hline $\mathrm{C}$ & -0.09233900 & -4.91152500 & 7.11209200 \\
\hline $\mathrm{C}$ & -2.02445300 & -1.31927200 & 1.49871100 \\
\hline $\mathrm{C}$ & -3.14187200 & -2.03474500 & 1.94051800 \\
\hline $\mathrm{C}$ & -4.27774100 & -2.11000300 & 1.15110900 \\
\hline $\mathrm{C}$ & -4.31491700 & -1.41340900 & -0.04691200 \\
\hline $\mathrm{C}$ & -3.22708100 & -0.61435100 & -0.41377600 \\
\hline $\mathrm{C}$ & -3.33252200 & 0.32181400 & -1.64379200 \\
\hline $\mathrm{C}$ & -4.71687200 & 0.15746400 & -2.28839600 \\
\hline $\mathrm{C}$ & -1.68713900 & 3.45325400 & -0.48555400 \\
\hline $\mathrm{C}$ & -2.62672700 & 4.45734700 & -0.64333700 \\
\hline $\mathrm{C}$ & -3.90245000 & 4.08613600 & -1.05980100 \\
\hline $\mathrm{C}$ & -4.15791100 & 2.75144400 & -1.34663500 \\
\hline $\mathrm{C}$ & -3.14955100 & 1.79025500 & -1.19828600 \\
\hline $\mathrm{C}$ & -2.23239300 & 0.09710200 & -2.71567700 \\
\hline $\mathrm{C}$ & -2.52356400 & 0.17619100 & -4.05155000 \\
\hline $\mathrm{C}$ & -1.42638800 & 0.28726800 & -4.98048100 \\
\hline $\mathrm{C}$ & -0.14809700 & 0.27883100 & -4.56278100 \\
\hline $\mathrm{C}$ & 0.21311900 & 0.05514000 & -3.12667000 \\
\hline B & -0.65077600 & -1.22950500 & 2.40552200 \\
\hline $\mathrm{H}$ & 2.37051600 & -1.42883200 & -1.10224400 \\
\hline $\mathrm{H}$ & 3.29390100 & -3.29181900 & 0.69067600 \\
\hline
\end{tabular}




\begin{tabular}{|c|c|c|c|}
\hline $\mathrm{H}$ & 1.51460500 & -3.10961400 & 2.76937100 \\
\hline $\mathrm{H}$ & 0.00491600 & 3.32307600 & 1.89257000 \\
\hline $\mathrm{H}$ & -0.94580000 & 3.08594600 & 4.45208200 \\
\hline $\mathrm{H}$ & -1.28694100 & 0.37808800 & 4.74570600 \\
\hline $\mathrm{H}$ & -1.48510700 & -3.90126100 & 2.78081500 \\
\hline $\mathrm{H}$ & -1.22767800 & -5.41164200 & 4.67134900 \\
\hline $\mathrm{H}$ & 0.79437800 & -2.33219000 & 6.86182100 \\
\hline $\mathrm{H}$ & 0.54251200 & -0.81854200 & 4.97483600 \\
\hline $\mathrm{H}$ & 0.02610600 & -5.95024200 & 6.78841800 \\
\hline $\mathrm{H}$ & 0.77105400 & -4.64813800 & 7.73033900 \\
\hline $\mathrm{H}$ & -0.97884000 & -4.87260500 & 7.75747400 \\
\hline $\mathrm{H}$ & -3.11063900 & -2.53336700 & 2.90068300 \\
\hline $\mathrm{H}$ & -5.13638200 & -2.69710700 & 1.46723300 \\
\hline $\mathrm{H}$ & -5.19741500 & -1.47740500 & -0.66640100 \\
\hline $\mathrm{H}$ & -5.52219500 & 0.32875300 & -1.57017500 \\
\hline $\mathrm{H}$ & -4.82296300 & -0.84628900 & -2.70491700 \\
\hline $\mathrm{H}$ & -4.84451200 & 0.86784300 & -3.10447900 \\
\hline $\mathrm{H}$ & -0.67275100 & 3.69036600 & -0.17886200 \\
\hline $\mathrm{H}$ & -2.36420700 & 5.49021000 & -0.44255300 \\
\hline $\mathrm{H}$ & -4.68675800 & 4.82802600 & -1.17907100 \\
\hline $\mathrm{H}$ & -5.14006700 & 2.46541900 & -1.69756900 \\
\hline $\mathrm{H}$ & 0.79197700 & -0.87534000 & -3.01892900 \\
\hline $\mathrm{H}$ & -3.53411000 & 0.24334400 & -4.42583600 \\
\hline $\mathrm{H}$ & -1.65057000 & 0.43791200 & -6.03413800 \\
\hline $\mathrm{H}$ & 0.65772100 & 0.44632300 & -5.27026200 \\
\hline Sc & -0.27700800 & 0.61270700 & -0.41165300 \\
\hline$P$ & 1.28480800 & 1.51758800 & -2.34660300 \\
\hline $\mathrm{C}$ & 3.02223700 & 0.94150800 & -1.96825700 \\
\hline $\mathrm{C}$ & 3.65651200 & 1.41543900 & -0.77659300 \\
\hline $\mathrm{C}$ & 3.78707300 & 0.11554200 & -2.83722200 \\
\hline $\mathrm{C}$ & 4.93626200 & 0.96875000 & -0.44281800 \\
\hline $\mathrm{C}$ & 5.05981600 & -0.32302800 & -2.44847000 \\
\hline $\mathrm{C}$ & 5.63300100 & 0.07692800 & -1.25184400 \\
\hline $\mathrm{H}$ & 5.40158900 & 1.31995300 & 0.47299000 \\
\hline $\mathrm{H}$ & 5.61894600 & -0.97456600 & -3.11660000 \\
\hline $\mathrm{H}$ & 6.62009400 & -0.27471100 & -0.96346300 \\
\hline $\mathrm{C}$ & 2.99992300 & 2.46353900 & 0.11123300 \\
\hline $\mathrm{H}$ & 1.91693000 & 2.26426600 & 0.09719900 \\
\hline $\mathrm{C}$ & 3.32995900 & -0.25489500 & -4.24014600 \\
\hline $\mathrm{H}$ & 2.35140100 & 0.20148300 & -4.38671000 \\
\hline $\mathrm{C}$ & 4.25858500 & 0.36243800 & -5.29796100 \\
\hline $\mathrm{H}$ & 4.33246100 & 1.44650800 & -5.16977200 \\
\hline $\mathrm{H}$ & 5.27132600 & -0.05146400 & -5.23722700 \\
\hline $\mathrm{H}$ & 3.87906500 & 0.16276300 & -6.30691800 \\
\hline $\mathrm{C}$ & 3.19226500 & -1.76674900 & -4.45919600 \\
\hline $\mathrm{H}$ & 2.50961300 & -2.22207900 & -3.73427900 \\
\hline
\end{tabular}




$\begin{array}{lccc}\mathrm{H} & 2.80569900 & -1.97912800 & -5.46238300 \\ \mathrm{H} & 4.15911600 & -2.27469300 & -4.36543300 \\ \mathrm{C} & 3.44295900 & 2.43499100 & 1.57611800 \\ \mathrm{H} & 3.35451000 & 1.43294400 & 2.00626800 \\ \mathrm{H} & 4.47924100 & 2.76987500 & 1.69752400 \\ \mathrm{H} & 2.81987500 & 3.11259300 & 2.16950000 \\ \mathrm{C} & 3.19828400 & 3.86708400 & -0.48491800 \\ \mathrm{H} & 2.81410400 & 3.92076100 & -1.50777300 \\ \mathrm{H} & 2.68399100 & 4.62716900 & 0.11663600 \\ \mathrm{H} & 4.26384200 & 4.12238600 & -0.50800900\end{array}$

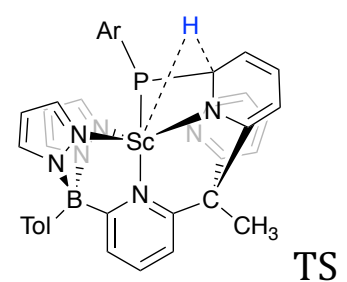

$\begin{array}{llcc}\mathrm{N} & 0.18970300 & -1.91561800 & 0.06740600 \\ \mathrm{~N} & -0.11157500 & -2.09671500 & 1.37395800 \\ \mathrm{~N} & -0.12811500 & 0.80414000 & 0.99728900 \\ \mathrm{~N} & -0.75463300 & 0.26779900 & 2.07318100 \\ \mathrm{~N} & -2.57692900 & -0.96384400 & -0.14762300 \\ \mathrm{~N} & -2.58420600 & 1.63153600 & -1.14785400 \\ \mathrm{~N} & -1.37937700 & 0.15705600 & -2.99823600 \\ \mathrm{C} & 1.08025100 & -2.85776300 & -0.26826400 \\ \mathrm{C} & 1.37157500 & -3.66282700 & 0.83473200 \\ \mathrm{C} & 0.58928300 & -3.14625700 & 1.85520600 \\ \mathrm{C} & 0.12083200 & 2.09017600 & 1.28520300 \\ \mathrm{C} & -0.37675300 & 2.41102900 & 2.54938900 \\ \mathrm{C} & -0.93720100 & 1.22471700 & 3.00428600 \\ \mathrm{C} & -1.33872800 & -1.84557300 & 3.58690500 \\ \mathrm{C} & -2.06922300 & -3.02015600 & 3.85213600 \\ \mathrm{C} & -2.07395100 & -3.63380400 & 5.10263500 \\ \mathrm{C} & -1.33166900 & -3.11206600 & 6.16720400 \\ \mathrm{C} & -0.55971700 & -1.97600800 & 5.91142200 \\ \mathrm{C} & -0.55841500 & -1.36999100 & 4.65527600 \\ \mathrm{C} & -1.36591500 & -3.74772100 & 7.53125100 \\ \mathrm{C} & -2.61212500 & -1.28661600 & 1.17688000 \\ \mathrm{C} & -3.84937900 & -1.54529000 & 1.78010200 \\ \mathrm{C} & -5.00960000 & -1.58152800 & 1.02168900 \\ \mathrm{C} & -4.94041500 & -1.27799300 & -0.33020200 \\ \mathrm{C} & -3.71024000 & -0.91550700 & -0.88077500 \\ \mathrm{C} & -3.65529500 & -0.27365400 & -2.28417400 \\ \mathrm{C} & -4.91391800 & -0.60476000 & -3.10124100 \\ \mathrm{C} & -2.47071400 & 2.92520500 & -0.84573300 \\ \mathrm{C} & -3.29428200 & 3.91414500 & -1.37881900\end{array}$




$\begin{array}{lrrr}\mathrm{C} & -4.28124100 & 3.52338300 & -2.27197200 \\ \mathrm{C} & -4.41567200 & 2.17127500 & -2.58185900 \\ \mathrm{C} & -3.55177200 & 1.24339600 & -2.00087700 \\ \mathrm{C} & -2.41312200 & -0.70584400 & -3.09448100 \\ \mathrm{C} & -2.35414400 & -1.81348700 & -3.91632500 \\ \mathrm{C} & -1.18615400 & -1.98704400 & -4.71514000 \\ \mathrm{C} & -0.08087400 & -1.21152900 & -4.51584600 \\ \mathrm{C} & -0.12776700 & -0.24228500 & -3.44145900 \\ \mathrm{~B} & -1.22425700 & -1.25174800 & 2.07648600 \\ \mathrm{H} & 1.45485300 & -2.89543000 & -1.28108800 \\ \mathrm{H} & 2.04678600 & -4.50361200 & 0.88571700 \\ \mathrm{H} & 0.47733700 & -3.46183900 & 2.88048000 \\ \mathrm{H} & 0.64791900 & 2.70525600 & 0.56763400 \\ \mathrm{H} & -0.32850500 & 3.35707900 & 3.06750300 \\ \mathrm{H} & -1.44118200 & 1.00173200 & 3.93217800 \\ \mathrm{H} & -2.63060900 & -3.49491900 & 3.05120200 \\ \mathrm{H} & -2.65684500 & -4.54098800 & 5.25065800 \\ \mathrm{H} & 0.06295600 & -1.56355100 & 6.70309700 \\ \mathrm{H} & 0.09932700 & -0.51827100 & 4.50628200 \\ \mathrm{H} & -1.50951800 & -4.83067700 & 7.46601800 \\ \mathrm{H} & -0.44059200 & -3.56070900 & 8.08435200 \\ \mathrm{H} & -2.19124700 & -3.34711400 & 8.13315100 \\ \mathrm{H} & -3.89180000 & -1.72560000 & 2.84693100 \\ \mathrm{H} & -5.96502300 & -1.82354600 & 1.48045400 \\ \mathrm{C} & -5.83945700 & -1.28083200 & -0.93213400 \\ \mathrm{H} & -5.81816200 & -0.16484700 & -2.67345000 \\ \mathrm{H} & -5.06174500 & -1.68581500 & -3.15563600 \\ \mathrm{H} & -4.27083600 & -0.52277300 & -3.97430300 \\ \mathrm{H} & -4.80374700 & -0.23417500 & -4.12299400 \\ \mathrm{H} & -1.68389100 & 3.17971900 & -0.14183100 \\ \mathrm{H} & -3.15334800 & 4.95312900 & -1.09980400 \\ \mathrm{H} & -4.94517100 & 4.25469700 & -2.72445900 \\ \mathrm{H} & -5.18243700 & 1.85512800 & -3.27791400 \\ \mathrm{H} & 0.15139300 & -1.33747900 & -2.24475700 \\ \mathrm{H} & -3.19517300 & -2.48742500 & -4.03058700 \\ \mathrm{H} & -1.17763400 & -2.76554900 & -5.47433300 \\ \mathrm{H} & 0.83969300 & -1.36739300 & -5.06557200 \\ \mathrm{H} & -2.54916400 & -0.12742100 & -1.02968300 \\ \mathrm{H} & & & \end{array}$




$\begin{array}{lccc}\mathrm{H} & 6.25466800 & -0.90512300 & -4.23520600 \\ \mathrm{C} & 3.31322400 & 0.26486300 & -0.80816200 \\ \mathrm{H} & 2.23943100 & 0.07547100 & -0.67955700 \\ \mathrm{C} & 2.27439000 & 0.95583700 & -5.78859700 \\ \mathrm{H} & 1.22816100 & 0.94758400 & -5.46867900 \\ \mathrm{C} & 2.65543200 & 2.42844100 & -6.00887400 \\ \mathrm{H} & 2.50203800 & 3.00754100 & -5.09351800 \\ \mathrm{H} & 3.70827500 & 2.51967700 & -6.30013900 \\ \mathrm{H} & 2.04248800 & 2.87412400 & -6.80106000 \\ \mathrm{C} & 2.36370100 & 0.17703600 & -7.10604200 \\ \mathrm{H} & 2.17062000 & -0.89199700 & -6.96714900 \\ \mathrm{H} & 1.62522100 & 0.56276300 & -7.81713100 \\ \mathrm{H} & 3.34601200 & 0.27635000 & -7.58156100 \\ \mathrm{C} & 4.05886800 & -0.67120700 & 0.14601600 \\ \mathrm{H} & 3.95134900 & -1.71985300 & -0.14572600 \\ \mathrm{H} & 5.12902500 & -0.43928500 & 0.19819400 \\ \mathrm{H} & 3.65727400 & -0.56300000 & 1.15875600 \\ \mathrm{C} & 3.56691200 & 1.72963600 & -0.41807100 \\ \mathrm{H} & 2.97394400 & 2.41332000 & -1.03406800 \\ \mathrm{H} & 3.31339300 & 1.90284300 & 0.63423700 \\ \mathrm{H} & 4.62326600 & 1.98616000 & -0.55942200\end{array}$

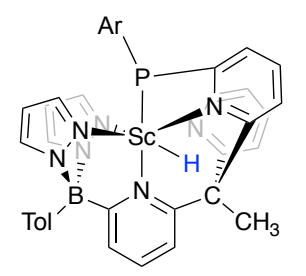

$\begin{array}{lccc}\mathrm{N} & 0.44004300 & -2.28681300 & 1.31212000 \\ \mathrm{~N} & -0.09177000 & -2.13352900 & 2.54635700 \\ \mathrm{~N} & 1.33703600 & 0.43865900 & 1.81725800 \\ \mathrm{~N} & 0.73546700 & 0.22573300 & 3.01631200 \\ \mathrm{~N} & -1.63238600 & -0.32933800 & 0.74114300 \\ \mathrm{~N} & -4.10528100 & 1.19302400 & -2.58218900 \\ \mathrm{~N} & -0.42187900 & 0.34344800 & -1.81479900 \\ \mathrm{C} & 0.69015000 & -3.59186900 & 1.14165500 \\ \mathrm{C} & 0.31210800 & -4.30471300 & 2.28124800 \\ \mathrm{C} & -0.18140300 & -3.33882300 & 3.14599900 \\ \mathrm{C} & 2.24280500 & 1.41094600 & 2.00170400 \\ \mathrm{C} & 2.22458700 & 1.84858200 & 3.32570700 \\ \mathrm{C} & 1.24154600 & 1.07732800 & 3.92846200 \\ \mathrm{C} & -1.03949000 & -0.94384400 & 4.60368300 \\ \mathrm{C} & -2.39629100 & -1.09115800 & 4.93541000 \\ \mathrm{C} & -2.81850600 & -1.39476200 & 6.22929900 \\ \mathrm{C} & -1.89992700 & -1.57600100 & 7.26684300 \\ \mathrm{C} & -0.54167500 & -1.47036900 & 6.94869200\end{array}$




\begin{tabular}{|c|c|c|c|}
\hline $\mathrm{C}$ & -0.12784400 & -1.17111100 & 5.65264800 \\
\hline $\mathrm{C}$ & -2.35282000 & -1.86391400 & 8.67340000 \\
\hline $\mathrm{C}$ & -1.61371400 & -0.02453900 & 2.06665500 \\
\hline $\mathrm{C}$ & -2.50848400 & 0.93528100 & 2.56089500 \\
\hline $\mathrm{C}$ & -3.44457000 & 1.51104100 & 1.71801200 \\
\hline $\mathrm{C}$ & -3.52894600 & 1.07876000 & 0.39804600 \\
\hline $\mathrm{C}$ & -2.60767400 & 0.14629000 & -0.07033200 \\
\hline C & -2.71175700 & -0.47200200 & -1.48333300 \\
\hline $\mathrm{C}$ & -2.60177000 & -2.01054000 & -1.33912800 \\
\hline C & -5.21842400 & 1.61381100 & -3.18018600 \\
\hline C & -6.34139300 & 0.80742600 & -3.36728300 \\
\hline $\mathrm{C}$ & -6.28714400 & -0.49723800 & -2.89137500 \\
\hline $\mathrm{C}$ & -5.12425700 & -0.94593600 & -2.26599000 \\
\hline C & -4.04281200 & -0.07041300 & -2.13598700 \\
\hline $\mathrm{C}$ & -1.56513700 & -0.03577300 & -2.40669200 \\
\hline $\mathrm{C}$ & -1.65514700 & -0.14687300 & -3.78700900 \\
\hline C & -0.51302400 & 0.12227600 & -4.55540600 \\
\hline C & 0.66097600 & 0.52459600 & -3.95218800 \\
\hline $\mathrm{C}$ & 0.69020500 & 0.66726900 & -2.54837900 \\
\hline B & -0.52160400 & -0.72388100 & 3.08183300 \\
\hline $\mathrm{H}$ & 1.11762600 & -3.92596500 & 0.20751700 \\
\hline $\mathrm{H}$ & 0.38521500 & -5.36750700 & 2.45667600 \\
\hline $\mathrm{H}$ & -0.58355300 & -3.42875500 & 4.14304700 \\
\hline $\mathrm{H}$ & 2.83792500 & 1.75162200 & 1.16751100 \\
\hline $\mathrm{H}$ & 2.83052200 & 2.61747700 & 3.78073200 \\
\hline $\mathrm{H}$ & 0.86425900 & 1.08915200 & 4.93942300 \\
\hline $\mathrm{H}$ & -3.15336700 & -0.98292200 & 4.16294800 \\
\hline $\mathrm{H}$ & -3.88243800 & -1.49892500 & 6.43386600 \\
\hline $\mathrm{H}$ & 0.20384300 & -1.63538900 & 7.72429900 \\
\hline $\mathrm{H}$ & 0.94039700 & -1.14095500 & 5.45018900 \\
\hline $\mathrm{H}$ & -3.31850400 & -2.37817100 & 8.68679200 \\
\hline $\mathrm{H}$ & -1.62916200 & -2.48643200 & 9.20860200 \\
\hline $\mathrm{H}$ & -2.47013400 & -0.93665300 & 9.24808100 \\
\hline $\mathrm{H}$ & -2.45782600 & 1.21679800 & 3.60697100 \\
\hline $\mathrm{H}$ & -4.13011500 & 2.27016200 & 2.08594800 \\
\hline $\mathrm{H}$ & -4.28407300 & 1.48156100 & -0.26155700 \\
\hline $\mathrm{H}$ & -3.31763800 & -2.39642400 & -0.60663000 \\
\hline $\mathrm{H}$ & -1.60171400 & -2.29495100 & -1.00303500 \\
\hline $\mathrm{H}$ & -2.77545400 & -2.49222600 & -2.30539600 \\
\hline $\mathrm{H}$ & -5.21417700 & 2.64672400 & -3.52532600 \\
\hline $\mathrm{H}$ & -7.22434900 & 1.19432000 & -3.86665200 \\
\hline $\mathrm{H}$ & -7.13608000 & -1.16567800 & -3.00613400 \\
\hline $\mathrm{H}$ & -5.06794100 & -1.96257700 & -1.89484500 \\
\hline $\mathrm{H}$ & 1.08341100 & -1.96556700 & -1.13530100 \\
\hline $\mathrm{H}$ & -2.58414500 & -0.44310400 & -4.25881600 \\
\hline $\mathrm{H}$ & -0.55906200 & 0.01618200 & -5.63670200 \\
\hline
\end{tabular}




$\begin{array}{lccc}\mathrm{H} & 1.55659600 & 0.72992800 & -4.52759200 \\ \mathrm{Sc} & 0.56238700 & -0.57148600 & -0.07474200 \\ \mathrm{P} & 2.03555300 & 1.22494300 & -1.50173500 \\ \mathrm{C} & 3.54759500 & 1.09614400 & -2.57494100 \\ \mathrm{C} & 4.45130000 & 0.00619500 & -2.48739400 \\ \mathrm{C} & 3.86135300 & 2.19717200 & -3.41836800 \\ \mathrm{C} & 5.63904800 & 0.04525200 & -3.22781700 \\ \mathrm{C} & 5.05581700 & 2.18304100 & -4.14611100 \\ \mathrm{C} & 5.94413800 & 1.11946800 & -4.05210400 \\ \mathrm{H} & 6.33655300 & -0.78505500 & -3.15563200 \\ \mathrm{H} & 5.29857300 & 3.02461700 & -4.78962900 \\ \mathrm{H} & 6.87177100 & 1.12883600 & -4.61920900 \\ \mathrm{C} & 4.20558700 & -1.19989000 & -1.59602700 \\ \mathrm{H} & 3.14934000 & -1.17860100 & -1.30347300 \\ \mathrm{C} & 2.96859800 & 3.42813300 & -3.53244800 \\ \mathrm{H} & 2.00087100 & 3.17443400 & -3.08671600 \\ \mathrm{C} & 3.54929700 & 4.58818300 & -2.71141900 \\ \mathrm{H} & 3.65406800 & 4.30616100 & -1.65922000 \\ \mathrm{H} & 4.53882300 & 4.87824300 & -3.08312000 \\ \mathrm{H} & 2.89670100 & 5.46716500 & -2.76436700 \\ \mathrm{C} & 2.71019500 & 3.85779100 & -4.98193900 \\ \mathrm{H} & 2.31571600 & 3.03216800 & -5.58277900 \\ \mathrm{H} & 1.97750600 & 4.67187000 & -5.00651900 \\ \mathrm{H} & 3.61703100 & 4.22513200 & -5.47524300 \\ \mathrm{C} & 4.42314500 & -2.53450000 & -2.31776600 \\ \mathrm{H} & 3.83010100 & -2.58790800 & -3.23569700 \\ \mathrm{H} & 5.47368700 & -2.70327700 & -2.58150300 \\ \mathrm{H} & 4.11301600 & -3.36128700 & -1.66968500 \\ \mathrm{C} & 5.05437300 & -1.11829500 & -0.31927800 \\ \mathrm{H} & 4.85161700 & -0.19629500 & 0.23530600 \\ \mathrm{H} & 4.84134000 & -1.96512600 & 0.34318500 \\ \mathrm{H} & 6.12557400 & -1.13521200 & -0.55350000\end{array}$

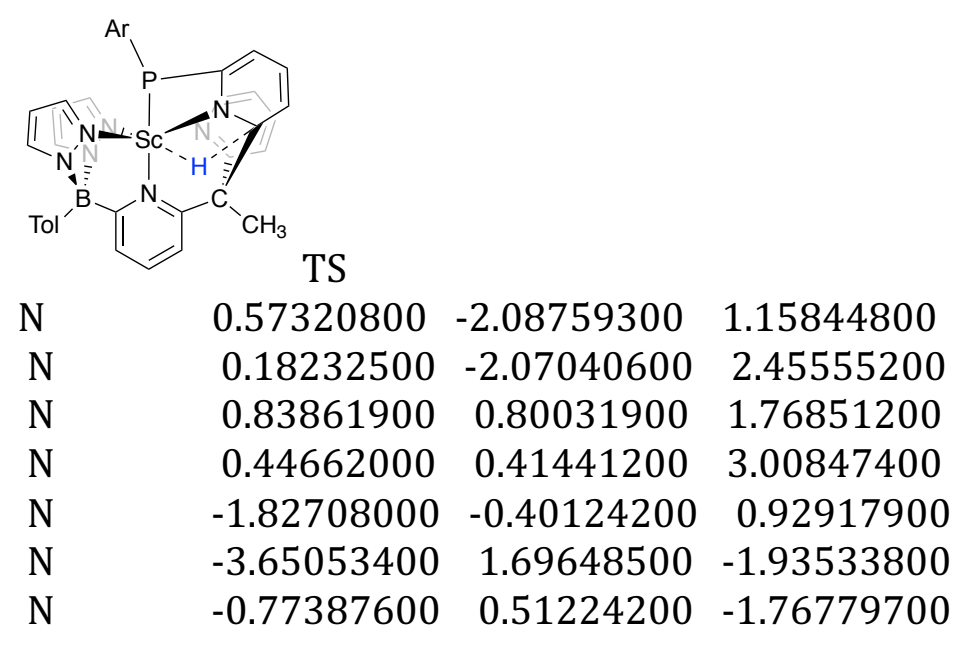




\begin{tabular}{|c|c|c|c|}
\hline $\mathrm{C}$ & 1.04400400 & -3.31268600 & 0.89578500 \\
\hline $\mathrm{C}$ & 0.97168200 & -4.11209800 & 2.03658800 \\
\hline $\mathrm{C}$ & 0.41252400 & -3.28496000 & 2.99905200 \\
\hline $\mathrm{C}$ & 1.42071400 & 2.00391300 & 1.89740800 \\
\hline $\mathrm{C}$ & 1.39323300 & 2.41466500 & 3.22975100 \\
\hline $\mathrm{C}$ & 0.75220000 & 1.38023500 & 3.89616100 \\
\hline $\mathrm{C}$ & -0.77055000 & -1.24354100 & 4.68225100 \\
\hline $\mathrm{C}$ & -1.96647200 & -1.84448400 & 5.11105300 \\
\hline $\mathrm{C}$ & -2.13839900 & -2.31839800 & 6.41091700 \\
\hline $\mathrm{C}$ & -1.11370200 & -2.22445700 & 7.35705600 \\
\hline $\mathrm{C}$ & 0.09818500 & -1.66555600 & 6.93818100 \\
\hline $\mathrm{C}$ & 0.26355400 & -1.19797800 & 5.63614300 \\
\hline $\mathrm{C}$ & -1.30831500 & -2.69851500 & 8.77257300 \\
\hline $\mathrm{C}$ & -1.84663500 & -0.35118700 & 2.29192900 \\
\hline $\mathrm{C}$ & -2.97042000 & 0.17965200 & 2.93013300 \\
\hline $\mathrm{C}$ & -4.07110200 & 0.58733600 & 2.18433500 \\
\hline $\mathrm{C}$ & -4.06453900 & 0.40779900 & 0.80930700 \\
\hline $\mathrm{C}$ & -2.91673700 & -0.09940400 & 0.19672900 \\
\hline C & -2.97351300 & -0.54381600 & -1.27082200 \\
\hline $\mathrm{C}$ & -3.50938500 & -1.99168000 & -1.19019300 \\
\hline $\mathrm{C}$ & -4.38406700 & 2.56205800 & -2.63269100 \\
\hline $\mathrm{C}$ & -5.41588500 & 2.18420000 & -3.49138100 \\
\hline $\mathrm{C}$ & -5.69235900 & 0.82717800 & -3.61312400 \\
\hline $\mathrm{C}$ & -4.92817400 & -0.08689900 & -2.89102000 \\
\hline $\mathrm{C}$ & -3.90361300 & 0.38790500 & -2.06552100 \\
\hline $\mathrm{C}$ & -1.62475300 & -0.53880400 & -2.03443600 \\
\hline $\mathrm{C}$ & -1.64015300 & -1.09706800 & -3.35936500 \\
\hline C & -0.52202600 & -0.93836400 & -4.13747000 \\
\hline $\mathrm{C}$ & 0.57696200 & -0.21419700 & -3.64706200 \\
\hline $\mathrm{C}$ & 0.41731300 & 0.53346300 & -2.47567400 \\
\hline B & -0.51465700 & -0.82262500 & 3.13817600 \\
\hline $\mathrm{H}$ & 1.39343700 & -3.55249000 & -0.09896900 \\
\hline $\mathrm{H}$ & 1.27235100 & -5.14270400 & 2.14954100 \\
\hline $\mathrm{H}$ & 0.16213300 & -3.48155300 & 4.02932600 \\
\hline $\mathrm{H}$ & 1.81037000 & 2.50191800 & 1.02131800 \\
\hline $\mathrm{H}$ & 1.78002400 & 3.33018100 & 3.65117900 \\
\hline $\mathrm{H}$ & 0.48651800 & 1.27665600 & 4.93691500 \\
\hline $\mathrm{H}$ & -2.78650600 & -1.97013700 & 4.40858600 \\
\hline $\mathrm{H}$ & -3.08462600 & -2.77677200 & 6.69218100 \\
\hline $\mathrm{H}$ & 0.92968800 & -1.60585800 & 7.63790200 \\
\hline $\mathrm{H}$ & 1.24012500 & -0.81322500 & 5.35184100 \\
\hline $\mathrm{H}$ & -2.04585300 & -3.50485600 & 8.82803400 \\
\hline $\mathrm{H}$ & -0.37230500 & -3.06406300 & 9.20590000 \\
\hline $\mathrm{H}$ & -1.66714600 & -1.88495300 & 9.41538100 \\
\hline $\mathrm{H}$ & -2.97299900 & 0.27444900 & 4.00988400 \\
\hline $\mathrm{H}$ & -4.94124300 & 1.01610700 & 2.67500900 \\
\hline
\end{tabular}




\begin{tabular}{|c|c|c|c|}
\hline $\mathrm{H}$ & -4.93106900 & 0.66272100 & 0.21135300 \\
\hline $\mathrm{H}$ & -4.49610800 & -2.01167100 & -0.71829400 \\
\hline $\mathrm{H}$ & -2.81931200 & -2.59001500 & -0.59041300 \\
\hline $\mathrm{H}$ & -3.58804800 & -2.46405700 & -2.17140800 \\
\hline $\mathrm{H}$ & -4.13648100 & 3.61358500 & -2.49551100 \\
\hline $\mathrm{H}$ & -5.98322100 & 2.93154800 & -4.03727300 \\
\hline $\mathrm{H}$ & -6.49252300 & 0.47862400 & -4.26058100 \\
\hline $\mathrm{H}$ & -5.13338900 & -1.14806600 & -2.97352500 \\
\hline $\mathrm{H}$ & -0.72427200 & -1.66891600 & -1.17773000 \\
\hline $\mathrm{H}$ & -2.47462600 & -1.69826200 & -3.69635800 \\
\hline $\mathrm{H}$ & -0.47049200 & -1.40058700 & -5.12085600 \\
\hline $\mathrm{H}$ & 1.51322000 & -0.16605200 & -4.19089400 \\
\hline Sc & 0.18195500 & -0.30705400 & -0.09737300 \\
\hline $\mathrm{P}$ & 1.66994700 & 1.44694900 & -1.55523900 \\
\hline $\mathrm{C}$ & 3.24247000 & 1.07661200 & -2.47608700 \\
\hline $\mathrm{C}$ & 4.22339900 & 0.18380200 & -1.97601600 \\
\hline $\mathrm{C}$ & 3.51897000 & 1.81617100 & -3.65918400 \\
\hline $\mathrm{C}$ & 5.43913400 & 0.04612100 & -2.65708700 \\
\hline $\mathrm{C}$ & 4.74444400 & 1.63882300 & -4.30852300 \\
\hline $\mathrm{C}$ & 5.70299100 & 0.76193500 & -3.81590600 \\
\hline $\mathrm{H}$ & 6.19232100 & -0.63527900 & -2.26918800 \\
\hline $\mathrm{H}$ & 4.95651600 & 2.20608400 & -5.21107100 \\
\hline $\mathrm{H}$ & 6.65256300 & 0.64113700 & -4.33120300 \\
\hline C & 4.03830800 & -0.63239800 & -0.70601700 \\
\hline $\mathrm{H}$ & 3.00359700 & -0.47311100 & -0.37371800 \\
\hline $\mathrm{C}$ & 2.54891500 & 2.84611600 & -4.22768400 \\
\hline $\mathrm{H}$ & 1.58311000 & 2.69987900 & -3.73384500 \\
\hline $\mathrm{C}$ & 3.02048400 & 4.26688100 & -3.88578100 \\
\hline $\mathrm{H}$ & 3.11099900 & 4.39762000 & -2.80305700 \\
\hline $\mathrm{H}$ & 3.99835200 & 4.47728900 & -4.33434300 \\
\hline $\mathrm{H}$ & 2.30936600 & 5.01218600 & -4.25995800 \\
\hline $\mathrm{C}$ & 2.31607700 & 2.69157700 & -5.73575300 \\
\hline $\mathrm{H}$ & 1.98553200 & 1.67930600 & -5.98791200 \\
\hline $\mathrm{H}$ & 1.54066000 & 3.39050000 & -6.06797500 \\
\hline $\mathrm{H}$ & 3.21719900 & 2.90687500 & -6.32089100 \\
\hline $\mathrm{C}$ & 4.22647700 & -2.13578600 & -0.94370500 \\
\hline $\mathrm{H}$ & 3.56501800 & -2.49691000 & -1.73809600 \\
\hline $\mathrm{H}$ & 5.25485800 & -2.37543900 & -1.23557300 \\
\hline $\mathrm{H}$ & 4.00751200 & -2.69688900 & -0.02867800 \\
\hline $\mathrm{C}$ & 4.95005700 & -0.13102300 & 0.42168800 \\
\hline $\mathrm{H}$ & 4.77814200 & 0.93009000 & 0.62623700 \\
\hline $\mathrm{H}$ & 4.76736300 & -0.69070300 & 1.34583700 \\
\hline $\mathrm{H}$ & 6.00721500 & -0.25248100 & 0.15885300 \\
\hline
\end{tabular}




$\begin{array}{llll} & & & \\ & & & \\ \end{array}$




\begin{tabular}{|c|c|c|c|}
\hline $\mathrm{H}$ & 0.64316100 & -3.39289900 & 3.64427500 \\
\hline $\mathrm{H}$ & 1.26127200 & 2.96949300 & 1.04547400 \\
\hline $\mathrm{H}$ & 1.13685000 & 3.58504200 & 3.73684300 \\
\hline $\mathrm{H}$ & 0.20728400 & 1.26376200 & 4.86547400 \\
\hline $\mathrm{H}$ & -2.50993500 & -2.42090600 & 4.15574600 \\
\hline $\mathrm{H}$ & -2.60231800 & -3.42652300 & 6.37809100 \\
\hline $\mathrm{H}$ & 1.19927500 & -1.68726900 & 7.33007200 \\
\hline $\mathrm{H}$ & 1.30681200 & -0.69618400 & 5.10376800 \\
\hline $\mathrm{H}$ & -1.36595300 & -4.14133900 & 8.42711200 \\
\hline $\mathrm{H}$ & 0.19922400 & -3.41299400 & 8.82445500 \\
\hline $\mathrm{H}$ & -1.29364900 & -2.52238200 & 9.12374300 \\
\hline $\mathrm{H}$ & -3.01922100 & -0.19177100 & 3.97665200 \\
\hline $\mathrm{H}$ & -5.12874700 & 0.42668300 & 2.80509000 \\
\hline $\mathrm{H}$ & -5.18925000 & 0.36254100 & 0.32143500 \\
\hline $\mathrm{H}$ & -5.17748700 & -1.27467200 & -1.13376800 \\
\hline $\mathrm{H}$ & -3.84135600 & -2.42404100 & -1.28995900 \\
\hline $\mathrm{H}$ & -4.45913700 & -1.56883300 & -2.71024500 \\
\hline $\mathrm{H}$ & -4.02824200 & 4.09222700 & -1.07026500 \\
\hline $\mathrm{H}$ & -4.71246800 & 4.38485600 & -3.45471100 \\
\hline $\mathrm{H}$ & -4.69885500 & 2.35667700 & -4.94579000 \\
\hline $\mathrm{H}$ & -3.99489900 & 0.17793200 & -3.99317200 \\
\hline $\mathrm{H}$ & -1.45224100 & -1.57980300 & -1.34205700 \\
\hline $\mathrm{H}$ & -2.72787200 & -1.82277000 & -3.78139400 \\
\hline $\mathrm{H}$ & -0.60621200 & -1.86206900 & -5.02514000 \\
\hline $\mathrm{H}$ & 1.45118500 & -0.77391600 & -4.04084700 \\
\hline Sc & 0.07312700 & -0.00401800 & -0.16915800 \\
\hline $\mathrm{P}$ & 1.47858400 & 1.45048600 & -1.84147500 \\
\hline $\mathrm{C}$ & 3.11624800 & 0.96437200 & -2.57601700 \\
\hline $\mathrm{C}$ & 4.14952700 & 0.37297800 & -1.80421900 \\
\hline $\mathrm{C}$ & 3.37834800 & 1.31876900 & -3.92779100 \\
\hline C & 5.39516900 & 0.12923400 & -2.39417300 \\
\hline $\mathrm{C}$ & 4.63660200 & 1.04784900 & -4.47533000 \\
\hline $\mathrm{C}$ & 5.64078400 & 0.45508400 & -3.72099200 \\
\hline $\mathrm{H}$ & 6.18696400 & -0.32270600 & -1.80343200 \\
\hline $\mathrm{H}$ & 4.83668700 & 1.31733400 & -5.50869200 \\
\hline $\mathrm{H}$ & 6.61368500 & 0.25509800 & -4.16304500 \\
\hline $\mathrm{C}$ & 3.97445000 & 0.04991300 & -0.32943100 \\
\hline $\mathrm{H}$ & 2.89527300 & -0.07115100 & -0.16025100 \\
\hline $\mathrm{C}$ & 2.36041400 & 2.05610400 & -4.79150200 \\
\hline $\mathrm{H}$ & 1.37609200 & 1.92088000 & -4.33464400 \\
\hline $\mathrm{C}$ & 2.66304600 & 3.56210600 & -4.78656600 \\
\hline $\mathrm{H}$ & 2.64838100 & 3.95805100 & -3.76661500 \\
\hline $\mathrm{H}$ & 3.65058500 & 3.76667200 & -5.21668400 \\
\hline $\mathrm{H}$ & 1.91711000 & 4.10933900 & -5.37435600 \\
\hline C & 2.26874100 & 1.52202100 & -6.22552400 \\
\hline $\mathrm{H}$ & 2.08867100 & 0.44275300 & -6.24054900 \\
\hline
\end{tabular}




$\begin{array}{lrrc}\mathrm{H} & 1.43988400 & 2.00849500 & -6.75113200 \\ \mathrm{H} & 3.17730300 & 1.72082100 & -6.80537400 \\ \mathrm{C} & 4.65874600 & -1.24654600 & 0.11565800 \\ \mathrm{H} & 4.39606500 & -2.08519300 & -0.53672800 \\ \mathrm{H} & 5.75083600 & -1.15646100 & 0.12007600 \\ \mathrm{H} & 4.35411300 & -1.50054300 & 1.13644500 \\ \mathrm{C} & 4.43368400 & 1.23182200 & 0.53655200 \\ \mathrm{H} & 3.89814000 & 2.14756500 & 0.26867700 \\ \mathrm{H} & 4.26016200 & 1.03175700 & 1.59996900 \\ \mathrm{H} & 5.50443200 & 1.41906900 & 0.39446600\end{array}$

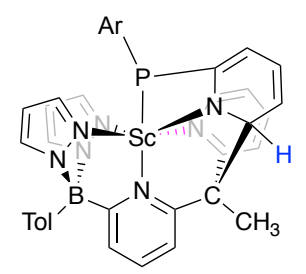

$\begin{array}{lrrr}\mathrm{N} & 0.70764000 & -0.98337700 & 1.81327600 \\ \mathrm{~N} & 0.03365800 & -0.80952200 & 2.97201700 \\ \mathrm{~N} & -0.37878900 & 1.71398700 & 1.41637500 \\ \mathrm{~N} & -1.05488400 & 1.47510600 & 2.56648700 \\ \mathrm{~N} & -2.02653600 & -0.80899900 & 0.64564000 \\ \mathrm{~N} & -1.37034100 & 0.72211100 & -1.69559600 \\ \mathrm{~N} & 0.10589400 & -1.66422400 & -1.28817700 \\ \mathrm{C} & 1.78986000 & -1.72646100 & 2.07534800 \\ \mathrm{C} & 1.82532200 & -2.04910300 & 3.43253900 \\ \mathrm{C} & 0.69358200 & -1.44763900 & 3.96270600 \\ \mathrm{C} & -0.40956700 & 3.03978500 & 1.21441100 \\ \mathrm{C} & -1.13897100 & 3.67308000 & 2.21983000 \\ \mathrm{C} & -1.54116400 & 2.63512900 & 3.04901200 \\ \mathrm{C} & -1.78228100 & -0.08859600 & 4.59749900 \\ \mathrm{C} & -2.50463000 & -1.18712900 & 5.09954300 \\ \mathrm{C} & -2.78527500 & -1.34099100 & 6.45557200 \\ \mathrm{C} & -2.34584300 & -0.40424200 & 7.39649400 \\ \mathrm{C} & -1.58483900 & 0.66870200 & 6.92419500 \\ \mathrm{C} & -1.30506700 & 0.81302800 & 5.56588900 \\ \mathrm{C} & -2.68187800 & -0.54519400 & 8.85698700 \\ \mathrm{C} & -2.40753400 & -0.58889900 & 1.93804400 \\ \mathrm{C} & -3.75116100 & -0.74949500 & 2.28761600 \\ \mathrm{C} & -4.66531700 & -1.22049000 & 1.35557500 \\ \mathrm{C} & -4.23297900 & -1.51221900 & 0.07199300 \\ \mathrm{C} & -2.90116100 & -1.26652700 & -0.28229600 \\ \mathrm{C} & -2.42955700 & -1.49388900 & -1.71894500 \\ \mathrm{C} & -3.46802300 & -2.29433800 & -2.50965900 \\ \mathrm{C} & -1.13818000 & 1.93131700 & -2.23172600 \\ \mathrm{C} & -1.65351200 & 2.34675800 & -3.44735200\end{array}$




\begin{tabular}{|c|c|c|c|}
\hline $\mathrm{C}$ & -2.44837900 & 1.44617100 & -4.15459900 \\
\hline $\mathrm{C}$ & -2.69570800 & 0.19590700 & -3.61208100 \\
\hline $\mathrm{C}$ & -2.15528300 & -0.15809100 & -2.36496000 \\
\hline C & -1.03369400 & -2.44156000 & -1.69611300 \\
\hline $\mathrm{C}$ & -0.81345300 & -3.15915800 & -2.98328700 \\
\hline $\mathrm{C}$ & 0.07077300 & -2.65789300 & -3.88335600 \\
\hline $\mathrm{C}$ & 0.93105500 & -1.56452100 & -3.55742700 \\
\hline $\mathrm{C}$ & 1.00155100 & -1.19533800 & -2.22769600 \\
\hline B & -1.32306600 & -0.01558300 & 3.04101600 \\
\hline $\mathrm{H}$ & 2.47233000 & -1.97139500 & 1.27266900 \\
\hline $\mathrm{H}$ & 2.56448000 & -2.63444900 & 3.95821000 \\
\hline $\mathrm{H}$ & 0.31715500 & -1.43469600 & 4.97312900 \\
\hline $\mathrm{H}$ & 0.10689000 & 3.47295900 & 0.368 \\
\hline $\mathrm{H}$ & -1.34084800 & 4.72697800 & 2.33774400 \\
\hline $\mathrm{H}$ & -2.13992600 & 2.65136500 & 3.9463 \\
\hline $\mathrm{H}$ & -2.83904400 & -1.96574700 & 4.41860400 \\
\hline $\mathrm{H}$ & -3.34785300 & -2.21082200 & 6.78944500 \\
\hline $\mathrm{H}$ & -1.19180700 & 1.39734300 & 7.63068600 \\
\hline $\mathrm{H}$ & -0.66641200 & 1.63940400 & 27300 \\
\hline $\mathrm{H}$ & -2.75699400 & -1.59637000 & 9.15192900 \\
\hline $\mathrm{H}$ & -1.92794000 & -0.06790900 & 9.49011900 \\
\hline $\mathrm{H}$ & -3.64570000 & -0.07425800 & 9.08751600 \\
\hline $\mathrm{H}$ & -4.07206700 & -0.50396000 & 244400 \\
\hline $\mathrm{H}$ & -5.70941500 & -1.35945100 & 1.62488100 \\
\hline $\mathrm{H}$ & -4.93623800 & -1.89225800 & 94900 \\
\hline $\mathrm{H}$ & -4.41502800 & -1.76080300 & -2.63606100 \\
\hline $\mathrm{H}$ & -3.67358600 & -3.24504200 & -2.00772600 \\
\hline $\mathrm{H}$ & -3.07212400 & -2.53336200 & -3.49827800 \\
\hline $\mathrm{H}$ & -0.50688700 & 2.59616100 & -1.65213400 \\
\hline $\mathrm{H}$ & -1.43428300 & 3.33996200 & -3.82274000 \\
\hline $\mathrm{H}$ & -2.87200600 & 1.71667100 & -5.11764300 \\
\hline $\mathrm{H}$ & -3.31363300 & -0.50784300 & -4.15296100 \\
\hline $\mathrm{H}$ & -1.28469200 & -3.15008600 & -0.89503500 \\
\hline $\mathrm{H}$ & -1.35861800 & -4.07758000 & -3.17678000 \\
\hline $\mathrm{H}$ & 0.19677400 & -3.15166400 & -4.84635000 \\
\hline $\mathrm{H}$ & 1.63483900 & -1.16697400 & -4.27815900 \\
\hline Sc & 0.02304000 & -0.03872100 & -0.03083300 \\
\hline $\mathrm{P}$ & 2.23313900 & -0.12774700 & -1.38591000 \\
\hline $\mathrm{C}$ & 3.24147200 & 0.71072800 & -2.68935900 \\
\hline $\mathrm{C}$ & 4.12249300 & -0.07295500 & -3.48640800 \\
\hline $\mathrm{C}$ & 3.26415200 & 2.12402900 & -2.82201100 \\
\hline $\mathrm{C}$ & 4.91817800 & 0.56100400 & -4.44595000 \\
\hline $\mathrm{C}$ & 4.09145200 & 2.71190900 & -3.78506400 \\
\hline $\mathrm{C}$ & 4.89931600 & 1.94036500 & -4.60870200 \\
\hline $\mathrm{H}$ & 5.58198100 & -0.03644700 & -5.06434100 \\
\hline $\mathrm{H}$ & 4.10610800 & 3.79325700 & -3.88965400 \\
\hline
\end{tabular}




$\begin{array}{llll}\mathrm{H} & 5.52839900 & 2.41299000 & -5.35882300 \\ \mathrm{C} & 4.32248200 & -1.56834800 & -3.26014300 \\ \mathrm{H} & 3.43771800 & -1.95187800 & -2.74629800 \\ \mathrm{C} & 2.47803100 & 3.03167000 & -1.89150100 \\ \mathrm{H} & 1.66173100 & 2.41529800 & -1.49199000 \\ \mathrm{C} & 3.34896900 & 3.45682800 & -0.69917000 \\ \mathrm{H} & 3.71768700 & 2.58464500 & -0.15085400 \\ \mathrm{H} & 4.21831800 & 4.03127400 & -1.03910000 \\ \mathrm{H} & 2.78434100 & 4.08420500 & 0.00122000 \\ \mathrm{C} & 1.86477800 & 4.25383000 & -2.58308800 \\ \mathrm{H} & 1.29285300 & 3.96691400 & -3.47122900 \\ \mathrm{H} & 1.19399400 & 4.78568000 & -1.89709100 \\ \mathrm{H} & 2.62513300 & 4.97775600 & -2.89606100 \\ \mathrm{C} & 4.48167900 & -2.38023100 & -4.55021700 \\ \mathrm{H} & 3.65669900 & -2.19904800 & -5.24608200 \\ \mathrm{H} & 5.41883300 & -2.15704700 & -5.07288600 \\ \mathrm{H} & 4.49367600 & -3.44993000 & -4.31536100 \\ \mathrm{C} & 5.52546100 & -1.78701900 & -2.32904700 \\ \mathrm{H} & 5.37840900 & -1.26974900 & -1.37596400 \\ \mathrm{H} & 5.66353200 & -2.85420200 & -2.12010600 \\ \mathrm{H} & 6.44844300 & -1.40793000 & -2.78323600\end{array}$

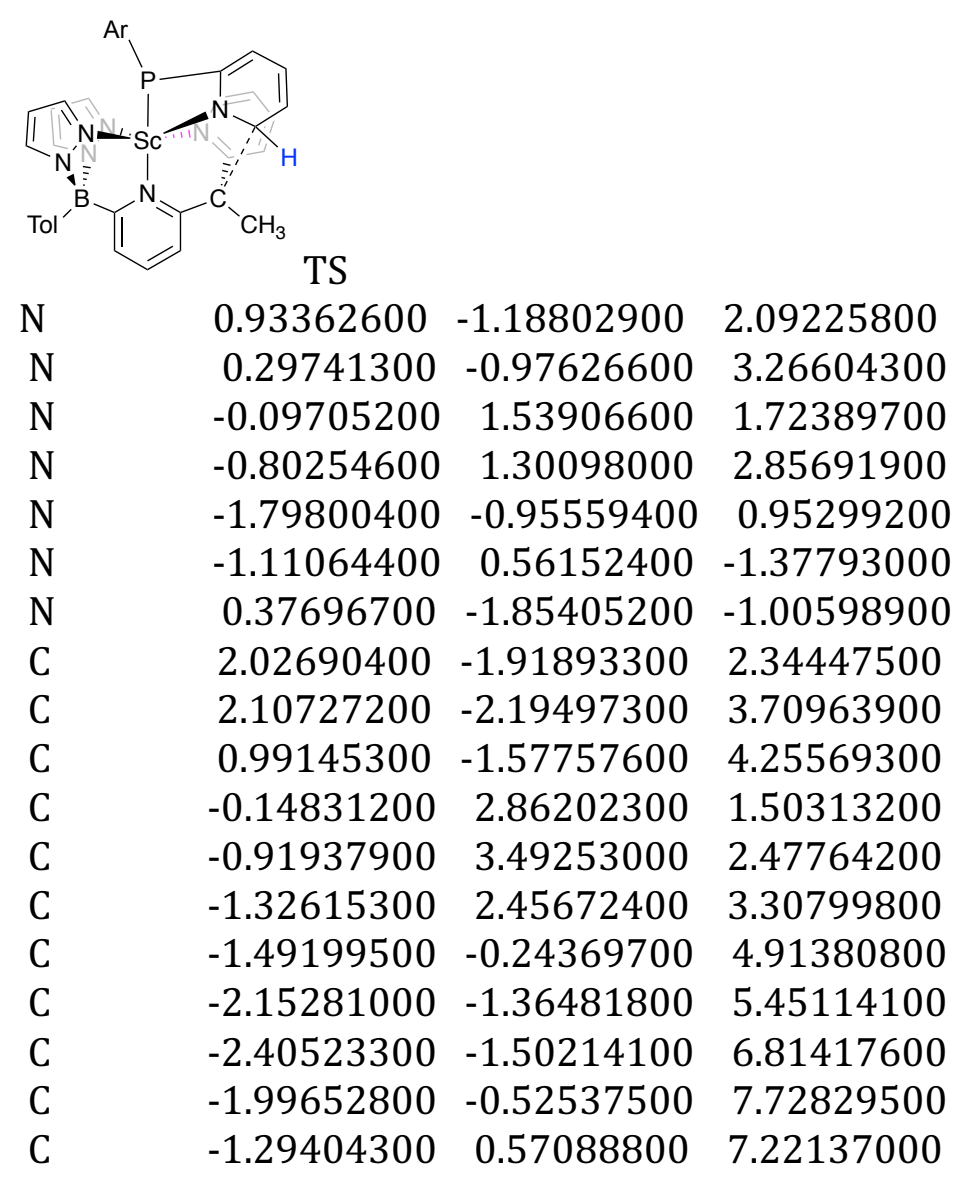




\begin{tabular}{|c|c|c|c|}
\hline $\mathrm{C}$ & -1.04242200 & 0.69882300 & 5.85562700 \\
\hline $\mathrm{C}$ & -2.30376900 & -0.65102300 & 9.19650400 \\
\hline $\mathrm{C}$ & -2.15309600 & -0.78554200 & 2.26407700 \\
\hline $\mathrm{C}$ & -3.46848000 & -1.03775200 & 2.65247000 \\
\hline $\mathrm{C}$ & -4.38114700 & -1.55782400 & 1.74056700 \\
\hline $\mathrm{C}$ & -3.97817100 & -1.79128800 & 0.43967000 \\
\hline $\mathrm{C}$ & -2.67924300 & -1.43728900 & 0.03621600 \\
\hline $\mathrm{C}$ & -2.28342900 & -1.58081900 & -1.40357100 \\
\hline $\mathrm{C}$ & -3.27413800 & -2.40685500 & -2.21019500 \\
\hline $\mathrm{C}$ & -0.81642800 & 1.75117600 & -1.93392200 \\
\hline $\mathrm{C}$ & -1.29354300 & 2.17078700 & -3.16012700 \\
\hline $\mathrm{C}$ & -2.12378100 & 1.29332500 & -3.86449500 \\
\hline $\mathrm{C}$ & -2.43983500 & 0.07020800 & -3.30746100 \\
\hline $\mathrm{C}$ & -1.94018900 & -0.29430200 & -2.03722800 \\
\hline $\mathrm{C}$ & -0.69936400 & -2.65931400 & -1.41375000 \\
\hline $\mathrm{C}$ & -0.54384200 & -3.31368800 & -2.70565800 \\
\hline C & 0.34390200 & -2.79705200 & -3.60993900 \\
\hline $\mathrm{C}$ & 1.23492700 & -1.75337800 & -3.25659000 \\
\hline $\mathrm{C}$ & 1.29094600 & -1.38040900 & -1.92100000 \\
\hline B & -1.06233500 & -0.18693300 & 3.34752800 \\
\hline $\mathrm{H}$ & 2.68705200 & -2.18624700 & 1.53056600 \\
\hline $\mathrm{H}$ & 2.86488500 & -2.76062600 & 4.23059600 \\
\hline $\mathrm{H}$ & 0.64784800 & -1.53126700 & 5.27680000 \\
\hline $\mathrm{H}$ & 0.38205900 & 3.29446000 & 0.66574600 \\
\hline $\mathrm{H}$ & -1.14549700 & 4.54351400 & 2.57572900 \\
\hline $\mathrm{H}$ & -1.95195300 & 2.47273700 & 4.18681100 \\
\hline $\mathrm{H}$ & -2.45717800 & -2.17389800 & 4.79226600 \\
\hline $\mathrm{H}$ & -2.92039300 & -2.39029300 & 7.17527800 \\
\hline $\mathrm{H}$ & -0.92466000 & 1.33211600 & 7.90591700 \\
\hline $\mathrm{H}$ & -0.44783000 & 1.54731100 & 5.52832200 \\
\hline $\mathrm{H}$ & -2.30023400 & -1.69678800 & 9.51921500 \\
\hline $\mathrm{H}$ & -1.57806200 & -0.10507700 & 9.80663100 \\
\hline $\mathrm{H}$ & -3.29597400 & -0.24407400 & 9.42840900 \\
\hline $\mathrm{H}$ & -3.77260600 & -0.83110000 & 3.67076900 \\
\hline $\mathrm{H}$ & -5.40343600 & -1.77194800 & 2.04271800 \\
\hline $\mathrm{H}$ & -4.68240000 & -2.19075400 & -0.27746900 \\
\hline $\mathrm{H}$ & -4.24387900 & -1.91385900 & -2.35080400 \\
\hline $\mathrm{H}$ & -3.45333600 & -3.37094000 & -1.72335200 \\
\hline $\mathrm{H}$ & -2.85845900 & -2.62495800 & -3.19672800 \\
\hline $\mathrm{H}$ & -0.15913500 & 2.39424700 & -1.35849800 \\
\hline $\mathrm{H}$ & -1.02263000 & 3.14573000 & -3.54883900 \\
\hline $\mathrm{H}$ & -2.52420200 & 1.56959100 & -4.83598900 \\
\hline $\mathrm{H}$ & -3.09422300 & -0.60628500 & -3.84041200 \\
\hline $\mathrm{H}$ & -1.04459200 & -3.31137900 & -0.60749500 \\
\hline $\mathrm{H}$ & -1.14216200 & -4.18802700 & -2.93831400 \\
\hline $\mathrm{H}$ & 0.42304400 & -3.24191100 & -4.60077700 \\
\hline
\end{tabular}




\begin{tabular}{|c|c|c|c|}
\hline $\mathrm{H}$ & 1.94366600 & -1.35018600 & -3.96941700 \\
\hline Sc & 0.23263600 & -0.20504300 & 0.27274400 \\
\hline $\mathrm{P}$ & 2.50144500 & -0.32419300 & -1.05120300 \\
\hline $\mathrm{C}$ & 3.52908800 & 0.52878400 & -2.33293600 \\
\hline $\mathrm{C}$ & 4.41663300 & -0.24741000 & -3.13102400 \\
\hline $\mathrm{C}$ & 3.56390000 & 1.94364400 & -2.44321900 \\
\hline $\mathrm{C}$ & 5.22313700 & 0.39574500 & -4.07528900 \\
\hline $\mathrm{C}$ & 4.40186800 & 2.54068800 & -3.39112800 \\
\hline $\mathrm{C}$ & 5.21133400 & 1.77709200 & -4.22040000 \\
\hline $\mathrm{H}$ & 5.89076500 & -0.19669800 & -4.69443500 \\
\hline $\mathrm{H}$ & 4.42390800 & 3.62346100 & -3.47796900 \\
\hline $\mathrm{H}$ & 5.84835000 & 2.25691200 & -4.95909500 \\
\hline $\mathrm{C}$ & 4.61786000 & -1.74497700 & -2.91920700 \\
\hline $\mathrm{H}$ & 3.73566200 & -2.13584900 & -2.40669900 \\
\hline $\mathrm{C}$ & 2.78322700 & 2.84547800 & -1.50234600 \\
\hline $\mathrm{H}$ & 1.97056600 & 2.22784700 & -1.09841800 \\
\hline $\mathrm{C}$ & 3.66380700 & 3.26820900 & -0.31595400 \\
\hline $\mathrm{H}$ & 4.04457900 & 2.39546300 & 0.22308600 \\
\hline $\mathrm{H}$ & 4.52583300 & 3.85030900 & -0.66121800 \\
\hline $\mathrm{H}$ & 3.10211100 & 3.88728200 & 0.39384100 \\
\hline $\mathrm{C}$ & 2.16450700 & 4.06966900 & -2.18594600 \\
\hline $\mathrm{H}$ & 1.58895900 & 3.78557200 & -3.07251000 \\
\hline $\mathrm{H}$ & 1.49655900 & 4.59869700 & -1.49515800 \\
\hline $\mathrm{H}$ & 2.92342200 & 4.79470200 & -2.49971700 \\
\hline $\mathrm{C}$ & 4.77945500 & -2.54594200 & -4.21598100 \\
\hline $\mathrm{H}$ & 3.95677100 & -2.35913500 & -4.91301400 \\
\hline $\mathrm{H}$ & 5.71753200 & -2.31836200 & -4.73500900 \\
\hline $\mathrm{H}$ & 4.79142900 & -3.61755900 & -3.99014900 \\
\hline $\mathrm{C}$ & 5.82089100 & -1.96987700 & -1.98945700 \\
\hline $\mathrm{H}$ & 5.67369600 & -1.45902700 & -1.03300600 \\
\hline $\mathrm{H}$ & 5.95971000 & -3.03832300 & -1.78766300 \\
\hline $\mathrm{H}$ & 6.74358200 & -1.58713200 & -2.44096800 \\
\hline $\mathrm{N}$ & -0.08175200 & -1.65679300 & 0.96401600 \\
\hline $\mathrm{N}$ & -0.24522400 & -1.46460400 & 2.29484000 \\
\hline $\mathrm{N}$ & -0.13311600 & 1.29057500 & 1.31958000 \\
\hline $\mathrm{N}$ & -0.66454100 & 1.05221000 & 2.54151300 \\
\hline $\mathrm{N}$ & -2.63310900 & -0.37763300 & 0.69896800 \\
\hline $\mathrm{N}$ & -1.97330200 & 1.28122100 & -1.57010500 \\
\hline $\mathrm{N}$ & -0.26200200 & -1.29928600 & -2.14681200 \\
\hline
\end{tabular}




\begin{tabular}{|c|c|c|c|}
\hline C & 0.77401300 & -2.67372700 & 0.80459000 \\
\hline $\mathrm{C}$ & 1.17658800 & -3.16132800 & 2.04744100 \\
\hline $\mathrm{C}$ & 0.50798800 & -2.36204100 & 2.96368500 \\
\hline $\mathrm{C}$ & 0.19266600 & 2.59238300 & 1.27369600 \\
\hline $\mathrm{C}$ & -0.16147400 & 3.22045200 & 2.46574100 \\
\hline $\mathrm{C}$ & -0.71495600 & 2.20415700 & 3.23534400 \\
\hline $\mathrm{C}$ & -1.28342100 & -0.66277200 & 4.48467500 \\
\hline $\mathrm{C}$ & -2.03448000 & -1.73292300 & 5.01001200 \\
\hline $\mathrm{C}$ & -2.00098200 & -2.08095800 & 6.35787400 \\
\hline $\mathrm{C}$ & -1.19690100 & -1.38399400 & 7.26604400 \\
\hline $\mathrm{C}$ & -0.40270100 & -0.35451300 & 6.75596000 \\
\hline $\mathrm{C}$ & -0.44041700 & -0.01399800 & 5.40349600 \\
\hline $\mathrm{C}$ & -1.19030200 & -1.72954800 & 8.73115800 \\
\hline C & -2.66451200 & -0.48549400 & 2.06691000 \\
\hline $\mathrm{C}$ & -3.87520200 & -0.68543700 & 2.71276900 \\
\hline $\mathrm{C}$ & -5.03774800 & -0.90701000 & 1.96158600 \\
\hline C & -4.97511900 & -0.89025500 & 0.59054900 \\
\hline C & -3.75886800 & -0.55306600 & -0.06517900 \\
\hline $\mathrm{C}$ & -3.77557500 & -0.36080400 & -1.49573600 \\
\hline $\mathrm{C}$ & -4.87816100 & -1.05762600 & -2.26591700 \\
\hline $\mathrm{C}$ & -1.48947400 & 2.43730500 & -2.08535800 \\
\hline $\mathrm{C}$ & -1.96325900 & 3.02501800 & -3.23180400 \\
\hline $\mathrm{C}$ & -2.98213300 & 2.34004700 & -3.93233700 \\
\hline $\mathrm{C}$ & -3.51419300 & 1.18827500 & -3.41785700 \\
\hline C & -3.07385200 & 0.67444400 & -2.14983100 \\
\hline $\mathrm{C}$ & -0.95587900 & -2.23647100 & -2.81144700 \\
\hline $\mathrm{C}$ & -0.50089700 & -2.81369100 & -3.98391600 \\
\hline $\mathrm{C}$ & 0.73945200 & -2.38218000 & -4.48574600 \\
\hline $\mathrm{C}$ & 1.46437700 & -1.41544600 & -3.81761600 \\
\hline $\mathrm{C}$ & 0.95523100 & -0.86344900 & -2.61799300 \\
\hline B & -1.24393700 & -0.38340900 & 2.88467000 \\
\hline $\mathrm{H}$ & 1.05876800 & -2.98517000 & -0.19075100 \\
\hline $\mathrm{H}$ & 1.85191500 & -3.97708900 & 2.25700300 \\
\hline $\mathrm{H}$ & 0.51117500 & -2.38177800 & 4.04194800 \\
\hline $\mathrm{H}$ & 0.67469700 & 2.99339100 & 0.39277900 \\
\hline $\mathrm{H}$ & -0.03028900 & 4.25635700 & 2.73963000 \\
\hline $\mathrm{H}$ & -1.13770600 & 2.23249700 & 4.22766700 \\
\hline $\mathrm{H}$ & -2.64503800 & -2.33492900 & 4.34265300 \\
\hline $\mathrm{H}$ & -2.60325200 & -2.91695400 & 6.70890000 \\
\hline $\mathrm{H}$ & 0.26775500 & 0.18416000 & 7.42304300 \\
\hline $\mathrm{H}$ & 0.23859300 & 0.76164100 & 5.06126000 \\
\hline $\mathrm{H}$ & -1.34206900 & -2.80154400 & 8.89159800 \\
\hline $\mathrm{H}$ & -0.24670000 & -1.44507100 & 9.20641800 \\
\hline $\mathrm{H}$ & -1.99395800 & -1.20581000 & 9.26385600 \\
\hline $\mathrm{H}$ & -3.91341500 & -0.69635000 & 3.79414700 \\
\hline $\mathrm{H}$ & -5.98692300 & -1.08719700 & 2.46099500 \\
\hline
\end{tabular}




\begin{tabular}{|c|c|c|c|}
\hline $\mathrm{H}$ & -5.87193000 & -1.04090500 & 0.00270800 \\
\hline $\mathrm{H}$ & -5.80657600 & -0.47230100 & -2.35879100 \\
\hline $\mathrm{H}$ & -5.14093000 & -2.00985100 & -1.79648300 \\
\hline $\mathrm{H}$ & -4.55258500 & -1.29931600 & -3.28310200 \\
\hline $\mathrm{H}$ & -0.65595800 & 2.87337600 & -1.54280100 \\
\hline $\mathrm{H}$ & -1.53977600 & 3.95412500 & -3.59394300 \\
\hline $\mathrm{H}$ & -3.35976900 & 2.73590300 & -4.87183300 \\
\hline $\mathrm{H}$ & -4.32482100 & 0.69374300 & -3.93747700 \\
\hline $\mathrm{H}$ & -1.91343000 & -2.51075400 & -2.37526700 \\
\hline $\mathrm{H}$ & -1.08996200 & -3.57013100 & -4.48964200 \\
\hline $\mathrm{H}$ & 1.13196100 & -2.81260900 & -5.40416000 \\
\hline $\mathrm{H}$ & 2.42509800 & -1.07346300 & -4.18761700 \\
\hline Sc & -0.74516500 & -0.02250000 & -0.36953500 \\
\hline$P$ & 1.67523200 & 0.34222900 & -1.51917100 \\
\hline $\mathrm{C}$ & 3.24232600 & 0.92008400 & -2.30698600 \\
\hline $\mathrm{C}$ & 4.47803700 & 0.40246800 & -1.83489100 \\
\hline $\mathrm{C}$ & 3.23900100 & 1.89392300 & -3.33991800 \\
\hline $\mathrm{C}$ & 5.66950900 & 0.84930300 & -2.41682900 \\
\hline $\mathrm{C}$ & 4.45776300 & 2.30202900 & -3.89300200 \\
\hline $\mathrm{C}$ & 5.66563600 & 1.78661400 & -3.44109600 \\
\hline $\mathrm{H}$ & 6.61660200 & 0.45218600 & -2.05985000 \\
\hline $\mathrm{H}$ & 4.45839200 & 3.04278000 & -4.68908800 \\
\hline $\mathrm{H}$ & 6.60174700 & 2.11864100 & -3.88305800 \\
\hline $\mathrm{C}$ & 4.57349700 & -0.62596400 & -0.71717500 \\
\hline $\mathrm{H}$ & 3.54740300 & -0.84695600 & -0.39978500 \\
\hline $\mathrm{C}$ & 1.96791100 & 2.54072700 & -3.86819500 \\
\hline $\mathrm{H}$ & 1.12611200 & 2.09083400 & -3.33073100 \\
\hline $\mathrm{C}$ & 1.95381800 & 4.04696300 & -3.57284300 \\
\hline $\mathrm{H}$ & 2.06030600 & 4.24221900 & -2.50063800 \\
\hline $\mathrm{H}$ & 2.77077600 & 4.56796100 & -4.08473900 \\
\hline $\mathrm{H}$ & 1.01365400 & 4.49637600 & -3.91155400 \\
\hline $\mathrm{C}$ & 1.75639400 & 2.27203600 & -5.36395900 \\
\hline $\mathrm{H}$ & 1.73610500 & 1.19875300 & -5.57455600 \\
\hline $\mathrm{H}$ & 0.80222200 & 2.69656900 & -5.69461200 \\
\hline $\mathrm{H}$ & 2.54859000 & 2.72039500 & -5.97461400 \\
\hline $\mathrm{C}$ & 5.20084100 & -1.93846600 & -1.20507900 \\
\hline $\mathrm{H}$ & 4.63901400 & -2.35670400 & -2.04635400 \\
\hline $\mathrm{H}$ & 6.23708400 & -1.79739700 & -1.53320600 \\
\hline $\mathrm{H}$ & 5.20806500 & -2.68123300 & -0.39950200 \\
\hline $\mathrm{C}$ & 5.32177900 & -0.06929700 & 0.50110200 \\
\hline $\mathrm{H}$ & 4.84542700 & 0.84298700 & 0.87373400 \\
\hline $\mathrm{H}$ & 5.32736500 & -0.80392800 & 1.31397200 \\
\hline $\mathrm{H}$ & 6.36481500 & 0.17131700 & 0.26444000 \\
\hline
\end{tabular}

